\title{
Modelos lineares parciais aditivos generalizados com suavização por meio de $\mathrm{P}$-splines
}

\author{
Amanda Amorim Holanda
}

\author{
DISSERTAÇÃO APRESENTADA \\ $A O$ \\ Instituto De Matemática e EstatísticA \\ DA \\ UniversidADE DE SÃo PAUlo \\ PARA \\ OBTENÇÃO DO TÍTULO \\ DE \\ Mestre em CiÊnCIAS \\ Programa: Estatística \\ Orientador: Prof. Dr. Gilberto Alvarenga Paula
}




\section{Modelos lineares parciais aditivos generalizados com suavização por meio de P-splines}

Esta versão da dissertação contém as correções e alterações sugeridas pela Comissão Julgadora durante a defesa da versão original do trabalho, realizada em 03/05/2018. Uma cópia da versão original está disponível no

Instituto de Matemática e Estatística da Universidade de São Paulo.

Comissão Julgadora:

- Prof. Dr. Gilberto Alvarenga Paula (orientador) - IME-USP

- Prof. Dr. Cristian Marcelo Villegas Lobos - ESALQ-USP

- Prof. Dr. Roberto Ferreira Manghi - UFPE 
Aos meus pais, por me ensinarem tudo sobre dedicação, persistência e coragem.

Às minhas irmãs, por todo o apoio. 


\section{Agradecimentos}

Agradeço ao meu orientador Prof. Dr. Gilberto Alvarenga Paula por sua orientação, dedicação e ajuda. Pelo seu cuidado profissional e por sua presteza em todas as revisões deste trabalho, que foram fundamentais para o resultado obtido. Por ter contribuído, desde a graduação, de diversas maneiras para o grande aprendizado que obtive ao longo desses anos no IME-USP e, principalmente, durante o mestrado.

Agradeço também à todos os professores que dividiram comigo seus preciosos conhecimentos durante essa caminhada. Entre eles, em especial, agradeço ao Prof. Dr. Victor Fossaluza, que desde a graduação sempre se mostrou disposto a me ajudar e ensinar e, principalmente, por todas as dicas, orientação e conversas que foram essenciais durante o mestrado. Ao Prof. Dr. Carlos Alberto de Bragança Pereira, pela orientação durante a graduação, pela confiança depositada em mim, pela parceria profissional e por toda ajuda.

A minha banca examinadora: Prof. Dr. Cristian Marcelo Villegas Lobos e Prof. Dr. Roberto Ferreira Manghi pelas valiosas contribuições que colaboraram para o enriquecimento deste trabalho.

Aos meus grandes amigos Daniella Rolim, Karina Bigatini, Marina Ruiz, Renato Moretto, Rafaela Aragão, Fabiana Alves, Giovanna Alencar e William Okura por todos momentos felizes, de apoio e amizade. Sou grata ainda, aos meus amigos da energia: a Lilian Silva e ao Weslley Lima por todos os cafés, conversas e risadas; a Juliana Chade por todo apoio, pelos ensinamentos, pela parceria e amizade; ao Prof. Paulo Bedaque por compartilhar comigo todo seu conhecimento, me ensinar e ser um grande amigo e a equipe que faço parte, pela parceria e por todos os almoços. Agradeço também a todas as pessoas que de alguma forma fizeram parte de todo esse processo, que me incentivaram e torceram por mim.

Agradeço às minhas irmãs Thaís e Bruna, pela ajuda em todas as questões que envolviam física e programação durante a graduação, pela revisão cuidadosa que fizeram neste trabalho, pelo apoio e por toda a vida compartilhada.

Aos meus pais Maria e Amaro, por toda a dedicação, pelo carinho, cuidado e amor incondicional em todos os momentos. Por terem possibilitado todas as minhas conquistas e por me ensinarem o verdadeiro valor do conhecimento.

Por fim, agradeço a Deus por iluminar a minha jornada e tornar cada sonho possível. 


\section{Resumo}

HOLANDA, A. A. Modelos lineares parciais aditivos generalizados com suavização por meio de P-splines. 2018. 107 f. Dissertação (Mestrado) - Instituto de Matemática e Estatística, Universidade de São Paulo, São Paulo, 2018.

Neste trabalho apresentamos os modelos lineares parciais generalizados com uma variável explicativa contínua tratada de forma não paramétrica e os modelos lineares parciais aditivos generalizados com no mínimo duas variáveis explicativas contínuas tratadas de tal forma. São utilizados os P-splines para descrever a relação da variável resposta com as variáveis explicativas contínuas. Sendo assim, as funções de verossimilhança penalizadas, as funções escore penalizadas e as matrizes de informação de Fisher penalizadas são desenvolvidas para a obtenção das estimativas de máxima verossimilhança penalizadas por meio da combinação do algoritmo backfitting (Gauss-Seidel) e do processo iterativo escore de Fisher para os dois tipos de modelo. Em seguida, são apresentados procedimentos para a estimação do parâmetro de suavização, bem como dos graus de liberdade efetivos. Por fim, com o objetivo de ilustração, os modelos propostos são ajustados à conjuntos de dados reais.

Palavras-chave: modelos parcialmente lineares, modelos semiparamétricos, modelos lineares generalizados, modelos lineares parciais generalizados, modelos aditivos generalizados, $\mathrm{P}$-splines, splines, método de suavização. 


\section{Abstract}

HOLANDA, A. A. Generalized additive partial linear models with $\mathbf{P}$-splines smoothing. 2018. 107 f. Dissertação (Mestrado) - Instituto de Matemática e Estatística, Universidade de São Paulo, São Paulo, 2018.

In this work we present the generalized partial linear models with one continuous explanatory variable treated nonparametrically and the generalized additive partial linear models with at least two continuous explanatory variables treated in such a way. The P-splines are used to describe the relationship among the response and the continuous explanatory variables. Then, the penalized likelihood functions, penalized score functions and penalized Fisher information matrices are derived to obtain the penalized maximum likelihood estimators by the combination of the backfitting (Gauss-Seidel) algorithm and the Fisher escoring iterative method for the two types of model. In addition, we present ways to estimate the smoothing parameter as well as the effective degrees of freedom. Finally, for the purpose of illustration, the proposed models are fitted to real data sets.

Keywords: partial linear models, semiparametric models, generalized linear models, generalized partial linear models, generalized additive models, P-splines, splines, smoothing method. 


\section{Sumário}

Lista de Abreviaturas $\quad$ xi

Lista de Símbolos $\quad$ xiii

Lista de Figuras $\quad$ xv

Lista de Tabelas $\quad$ xvii

1 Introdução $\quad 1$

1.1 Considerações Preliminares . . . . . . . . . . . . . . . . . . . . . 1



1.2 .1 Garotas de Varsóvia . . . . . . . . . . . . . . . . . . . . . . 3

1.2 .2 Clientes de uma loja . . . . . . . . . . . . . . . . . 4

1.2 .3 Valor de aluguel em Munique . . . . . . . . . . . . . . . 5

2 Modelos lineares parciais generalizados $\quad 15$

2.1 Revisão dos modelos lineares generalizados . . . . . . . . . . . . . . . . . . 15

2.2 Especificação do modelo . . . . . . . . . . . . . . . . . . . . . 17

2.3 Método de suavização P-splines . . . . . . . . . . . . . . . . . 18



2.4 .1 Função de verossimilhança penalizada . . . . . . . . . . . . . . 24

2.4 .2 Função escore e matriz de informação de Fisher penalizadas . . . . . . . . . . 25

2.5 Estimação dos parâmetros do modelo . . . . . . . . . . . . . . . 26



2.5.2 Estimação do parâmetro de suavização . . . . . . . . . . . . . . . . 30

2.6 Resultados Assintóticos . . . . . . . . . . . . . . . . . . . 31

2.6 .1 Teste de hipóteses . . . . . . . . . . . . . . . . . . . 33

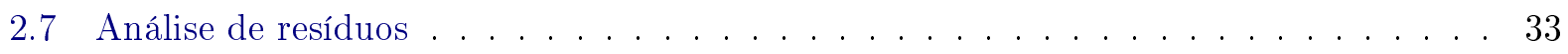

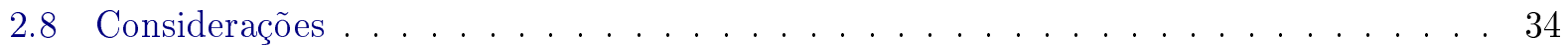

3 Modelos lineares parciais aditivos generalizados $\quad 35$

3.1 Especificação do modelo . . . . . . . . . . . . . . . . . . . 36

3.2 Critério de penalização . . . . . . . . . . . . . . . . . . 36

3.2 .1 Função de verossimilhança penalizada . . . . . . . . . . . . . . 37

3.2.2 Função escore e matriz de informação de Fisher penalizada . . . . . . . . . . 37

3.3 Estimação dos parâmetros . . . . . . . . . . . . . . . . . . . . . 39 
3.3.1 Graus de liberdade efetivos . . . . . . . . . . . . . . . . . 41

3.3.2 Estimação do parâmetro de suavização . . . . . . . . . . . . . . . . . . . 42

3.4 Teste de hipóteses . . . . . . . . . . . . . . . . . . . . . 43

3.5 Considerações . . . . . . . . . . . . . . . . . . . . . 4 44

4 Aplicações $\quad 45$

4.1 Garotas de Varsóvia . . . . . . . . . . . . . . . . . . . . . . . . 45

4.1 .1 Diagnóstico . . . . . . . . . . . . . . . . . . . . 47

4.2 Clientes de uma loja . . . . . . . . . . . . . . . . . . . . . . . 49

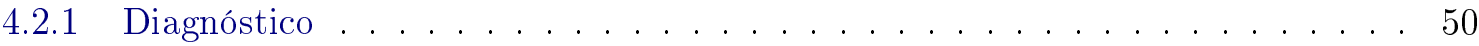

4.3 Valor de aluguel em Munique . . . . . . . . . . . . . . . . . . . . . 52

4.3 .1 Diagnóstico . . . . . . . . . . . . . . . . . . . . 61

5 Conclusões $\quad \mathbf{6 5}$

5.1 Considerações finais . . . . . . . . . . . . . . . . . . . 65

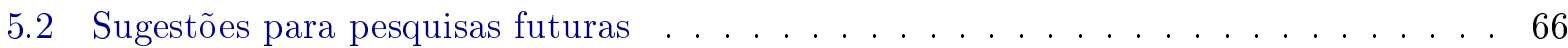

$\begin{array}{ll}\text { A Derivações do MLPG } & \mathbf{6 7}\end{array}$

A.1 Funções escore penalizadas . . . . . . . . . . . . . . . . . . 67

A.2 Matriz de informação de Fisher penalizada . . . . . . . . . . . . . . . 68

B Derivações do MLPAG

B.1 Funções escore penalizadas . . . . . . . . . . . . . . . . . . . 73

B.2 Matriz de informação de Fisher penalizada . . . . . . . . . . . . . . . . . . . . 74

$\begin{array}{ll}\text { C Derivação do Suavizador } & 81\end{array}$

C.1 MLPGs . . . . . . . . . . . . . . . . . . . . . . . 81

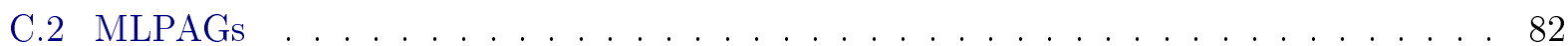

$\begin{array}{ll}\text { Referências Bibliográficas } & 85\end{array}$ 


\title{
Lista de Abreviaturas
}

\author{
AIC Critério de informação de Akaike \\ BIC Critério de informação Bayesiano \\ EMVP Estimador de máxima verossimilhança penalizada \\ GAIC Critério de informação de Akaike generalizado \\ GAM Generalized additive models \\ GAMLSS Generalized additive models for location, scale and shape \\ MAGs Modelos aditivos generalizados \\ MLPAGs Modelos lineares parciais aditivos generalizados \\ MLGs Modelos lineares generalizados \\ MLPGs Modelos lineares parciais generalizados \\ VC Validação cruzada \\ VCG Validação cruzada generalizada
}




\section{Lista de Símbolos}

$\begin{array}{ll}\mathbf{X}, \mathbf{W}, \mathbf{V}, \mathbf{H}, \mathbf{P} & \text { Matrizes reais } \\ \mathbf{y}, \mathbf{x}, \mathbf{b} & \text { Vetores constantes } \\ y_{i j}, x_{i j}, b_{i j} & \text { Valores das variáveis } \\ \beta, \gamma, \lambda, \phi & \text { Parâmetros } \\ \boldsymbol{\theta}, \boldsymbol{\beta}, \boldsymbol{\gamma}, \boldsymbol{\lambda} & \text { Vetores de parâmetros } \\ \mathbf{X}^{\top} & \text { Transposta da matriz } \mathbf{X} \\ \mathbf{X}^{-1} & \text { Inversa da matriz } \mathbf{X} \\ \operatorname{tr}\{\mathbf{X}\} & \text { Traço da matriz } \mathbf{X}\end{array}$




\section{Lista de Figuras}

1.1 Gráfico do logito da frequência relativa de ocorrência de Menarca versus idade média para os dados de Garotas de Varsóvia, ajustados pela tendência. . . . . . . . . . . . . 4

1.2 Gráficos boxplots robustos das variáveis número de clientes, número de domicílios e renda média referentes aos dados Clientes de uma Loja. . . . . . . . . . . . . . . . . 6

1.3 Gráficos boxplots robustos das variáveis idade, distância ao concorrente mais próximo e distância à loja referentes aos dados Clientes de uma Loja. . . . . . . . . . . . . . . 7

1.4 Gráficos de dispersão do $\log$ da variável número de clientes contra as variáveis explicativas referentes aos dados Clientes de uma Loja, ajustados pelas respectivas tendências. . . . . . . . . . . . . . . . . . . . . . . . 8

1.5 Gráfico de correlação entre as variáveis referentes aos dados Clientes de uma Loja. $\quad$ • 9

1.6 Gráfico da densidade do valor do aluguel para os dados Valor de Aluguel em Munique. 10

1.7 Gráficos de dispersão do log do valor do aluguel contra variáveis explicativas contínuas para os dados de Valor do Aluguel, ajustados pelas respectivas tendências. . . . . . . 11

1.8 Gráfico de correlação entre as variáveis quantitativas referentes aos dados Valor do Aluguel. . . . . . . . . . . . . . . . . . . . . . . . . . 12

1.9 Gráficos boxplots robustos do log do valor do aluguel segundo os níveis das variáveis explicativas qualitativas localização e instalações do banheiro para os dados de Valor do Aluguel. . . . . . . . . . . . . . . . . . . . . . . . . . . 13

1.10 Gráficos boxplots robustos do log do valor do aluguel segundo os níveis das variáveis explicativas qualitativas qualidade da cozinha e aquecimento central para os dados de Valor do Aluguel. . . . . . . . . . . . . . . . . . . . . . . . . . . . . . 14

2.1 Diagrama do esquema de Computação Triangular dos B-splines. . . . . . . . . . . . . 19

2.2 (a) B-spline de grau 1 considerando cinco nós. (b) B-splines de grau 1 considerando os mesmos cinco nós. . . . . . . . . . . . . . . . . . . . . . . . . . . 20

2.3 (a) B-spline quadrático considerando seis nós. (b) B-splines quadráticos considerando os mesmos seis nós. . . . . . . . . . . . . . . . . . . . . . . . . . . . . 21

2.4 (a) B-spline cúbico considerando onze nós. (b) B-splines cúbicos considerando os mesmos onze nós. . . . . . . . . . . . . . . . . . . . . . . . . . . . . 21

4.1 Banda de confiança pontual de $95 \%$ para a função $\mathrm{f}(\mathrm{t})$ para os dados Garotas de Varsóvia. . . . . . . . . . . . . . . . . . . . . . 46

4.2 Curva ajustada para o logito da proporção de meninas com menstruação já iniciada. 47

4.3 Gráficos de resíduos referentes ao modelo ajustado aos dados Garotas de Varsóvia. 48 
4.4 Bandas de confiança pontuais aproximadas de $95 \%$ para os efeitos das variáveis explicativas referentes ao modelo ajustado aos dados Clientes de uma Loja. . . . . . . . 50

4.5 Gráficos de resíduos referentes ao modelo ajustado aos dados Clientes de uma Loja. . 51

4.6 Bandas de confiança pontuais aproximadas de 95\% para os efeitos das variáveis explicativas área e ano de construção no valor médio do aluguel referentes ao modelo utilizando a distribuição gama ajustado aos dados Valor do Aluguel. . . . . . . . . . 53

4.7 Gráficos de resíduos referentes ao modelo utilizando a distribuição gama ajustado aos dados Valor do Aluguel. . . . . . . . . . . . . . . . . . . . . . 54

4.8 Bandas de confiança pontuais aproximadas de $95 \%$ para os efeitos das variáveis explicativas área e ano de construção no valor médio do aluguel referentes ao modelo duplo utilizando a distribuição gama ajustado aos dados Valor do Aluguel. . . . . . . 56

4.9 Bandas de confiança pontuais aproximadas de 95\% para os efeitos das variáveis explicativas área e ano de construção no coeficiente de variação do aluguel referentes ao modelo duplo utilizando a distribuição gama ajustado aos dados Valor do Aluguel. 56

4.10 Gráficos de resíduos referentes ao modelo duplo utilizando a distribuição gama ajustado aos dados Valor do Aluguel. . . . . . . . . . . . . . . . . . . 57

4.11 Bandas de confiança pontuais aproximadas de $95 \%$ para os efeitos das variáveis explicativas área e ano de construção no valor médio do aluguel referentes ao modelo utilizando a distribuição gama gemeralizada ajustado aos dados Valor do Aluguel. . . 59

4.12 Gráficos de resíduos referentes ao modelo utilizando a distribuição gama generalizada ajustado aos dados Valor do Aluguel. . . . . . . . . . . . . . . . . 60

4.13 Bandas de confiança pontuais aproximadas de 95\% para os efeitos das variáveis explicativas área e ano de construção no valor médio do aluguel referentes ao modelo duplo utilizando a distribuição gama generalizada ajustado aos dados Valor do Aluguel. 62

4.14 Bandas de confiança pontuais aproximadas de $95 \%$ para os efeitos das variáveis explicativas área e ano de construção no coeficiente de variação do aluguel referentes ao modelo duplo utilizando a distribuição gama generalizada ajustado aos dados Valor do Aluguel. . . . . . . . . . . . . . . . . . . . . . . . 6 63

4.15 Gráficos de resíduos referentes ao modelo duplo utilizando a distribuição gama generalizada ajustado aos dados Valor do Aluguel. . . . . . . . . . . . . . . . . . . . 64 


\section{Lista de Tabelas}

1.1 Ocorrência do início da menstruação nas garotas de Varsóvia. . . . . . . . . . . . . 3

2.1 Principais distribuições que pertencem à família exponencial. . . . . . . . . . . . . 16

2.2 Ligações canônicas mais comuns nos MLGs. . . . . . . . . . . . . . . . . 17

4.1 Estimativas de máxima verossimilhança penalizadas referentes ao modelo ajustado, seus respectivos erros padrão aproximados e valores-p, aos dados Garotas de Varsóvia 46

4.2 Estimativas de máxima verossimilhança penalizadas referentes ao modelo ajustado, seus respectivos erros padrão aproximados e valores-p, aos dados Clientes de uma Loja. 49

4.3 Estimativas de máxima verossimilhança penalizadas referentes ao modelo utilizando a distribuição gama ajustado, seus respectivos erros padrão aproximados e valores-p, aos dados Valor do Aluguel. . . . . . . . . . . . . . . . . . . . . . . 52

4.4 Estimativas de máxima verossimilhança penalizadas para os efeitos da localização referentes ao modelo duplo utilizando a distribuição gama ajustado, seus respectivos erros padrão aproximados e valores-p, aos dados Valor do Aluguel. . . . . . . . . . . 55

4.5 Estimativas de máxima verossimilhança penalizadas para os efeitos do coeficiente de variação referentes ao modelo duplo utilizando a distribuição gama ajustado, seus respectivos erros padrão aproximados e valores-p, aos dados Valor do Aluguel. . . . . 55

4.6 Estimativas de máxima verossimilhança penalizadas referentes ao modelo utilizando a distribuição gama generalizada ajustado, seus respectivos erros padrão aproximados e valores-p, aos dados Valor do Aluguel. . . . . . . . . . . . . . . . . . . . 58

4.7 Estimativas de máxima verossimilhança penalizadas para os efeitos da localização referentes ao modelo duplo utilizando a distribuição gama generalizada ajustado, seus respectivos erros padrão aproximados e valores-p, aos dados Valor do Aluguel. . 61

4.8 Estimativas de máxima verossimilhança penalizadas para os efeitos do coeficiente de variação referentes ao modelo duplo utilizando a distribuição gama generalizada ajustado, seus respectivos erros padrão aproximados e valores-p, aos dados Valor do Aluguel. . . . . . . . . . . . . . . . . . . . . . . . . . 62 
xviii LISTA DE TABELAS 


\section{Capítulo 1}

\section{Introdução}

\subsection{Considerações Preliminares}

Um dos principais desafios da modelagem de regressão é tentar identificar a verdadeira relação funcional entre a média da variável resposta (ou algum outro parâmetro de interesse) e cada variável explicativa.

Quando há um conhecimento prévio a respeito dessa relação funcional através, por exemplo, de estudos experimentais ou mesmo desenvolvimentos teóricos, sendo possível selecionar uma classe de curvas que acredita-se conter, mesmo que aproximadamente, a verdadeira relação entre a média da variável resposta e as variáveis explicativas, tem-se a metodologia conhecida como regressão paramétrica. Contudo, quando fica a critério da amostra indicar possíveis relações entre as variáveis, dado que não se tem nenhum conhecimento prévio à respeito delas, é usual selecionar um espaço flexível de funções que acredita-se conter a verdadeira relação funcional. Essa metodologia é conhecida como regressão não paramétrica (vide, por exemplo, Eubank 1999).

A regressão paramétrica, em geral, é mais simples do ponto de vista de estimação e inferência. Porém, a regressão não paramétrica permite captar aspectos locais da relação funcional com mais precisão, como também controlar de forma mais eficiente os efeitos das covariáveis.

Para ilustrar as duas metodologias considere o modelo de regressão

$$
y_{i}=f\left(x_{i}\right)+\epsilon_{i}
$$

em que $y_{i}$ e $x_{i}$ são, respectivamente, os valores observados da resposta e da variável explicativa $x$ (assumida contínua), $\epsilon_{i}$ são erros não correlacionados de média zero e variância constante, para $i=1, \ldots, n$, e $f(x)$ é uma função desconhecida.

Em um modelo de regressão paramétrica é assumida uma forma conhecida para $f(x)$, por exemplo, $f(x)=\beta_{1}+\beta_{2} x$ (relação linear), $f(x)=\beta_{1} x^{\beta_{2}}$ (relação linear em $\beta_{1}$ e não linear em $\beta_{2}$ ) e $f(x)=\beta_{1}+\beta_{2} \exp \left\{-\beta_{3} x^{\beta_{4}}\right\}$ (relação linear em $\beta_{1}$ e $\beta_{2}$ e não linear em $\beta_{3}$ e $\beta_{4}$ ), em que $\beta_{1}, \beta_{2}$, $\beta_{3}$ e $\beta_{4}$ são parâmetros desconhecidos e eventualmente interpretáveis. Por meio de procedimentos usuais, pode-se estimar e fazer inferência para esses parâmetros. Já na regressão não paramétrica, as suposições a respeito de $f(x)$ são mais gerais, como supor que $f(x)$ pertence ao espaço infinitodimensional de funções duplamente contínuas e diferenciáveis e duplamente integráveis.

Há vários procedimentos para estimar a função $f(x)$, dentre os mais conhecidos estão os métodos de suavização através de Kernel (Wand e Jones, 1995), de suavização polinomial local por meio de 
Kernel e de suavização por meio de splines (vide, por exemplo, Reinsch 1967 e Eubank 1999). Esses últimos são generalizações de polinômios cúbicos utilizados na regressão paramétrica. Os splines mais conhecidos são os splines cúbicos, os splines cúbicos naturais e os B-splines (vide, por exemplo, Hastie e Tibshirani 1990; Green e Silverman 1994 e Wood 2017).

Para ilustrar um exemplo de estimação de $f(x)$ no contexto não paramétrico, vamos supor o modelo (1.1), em que $a \leq x_{1}<x_{2}<\ldots<x_{n} \leq b$ com a função $f(x)$ pertencente ao espaço infinitodimensional de funções duplamente contínuas e diferenciáveis e duplamente integráveis. Considere o procedimento de estimação por mínimos quadrados penalizados, que consiste em minimizar a função objetivo

$$
\sum_{i=1}^{n}\left\{y_{i}-f\left(x_{i}\right)\right\}^{2}+\frac{\lambda}{2} \int_{a}^{b}\left[f^{\prime \prime}(x)\right]^{2} d x
$$

em que $\lambda>0$ é o parâmetro de suavização. Reinsch (1967) mostra que a solução para $f(x)$ em (1.2) é um spline cúbico natural com nós nos pontos $x_{1}, \ldots, x_{n}$.

Há variantes para o modelo (1.1). Por exemplo, se adicionarmos um componente paramétrico tal que $y_{i}=\mathbf{x}_{i}^{\top} \boldsymbol{\beta}+f\left(t_{i}\right)+\epsilon_{i}$, em que $\mathbf{x}_{i}=\left(x_{i 1}, \ldots, x_{i p}\right)^{\top}$ e $\boldsymbol{\beta}=\left(\beta_{1}, \ldots, \beta_{p}\right)^{\top}$, tem-se os modelos parcialmente lineares. Por outro lado, se temos apenas funções não paramétricas, em que $y_{i}=$ $f_{1}\left(t_{i 1}\right)+\ldots+f_{r}\left(t_{i r}\right)+\epsilon_{i}$, o modelo passa a ser chamado de modelo aditivo. Se adicionarmos um componente paramétrico a esse último, fazendo $y_{i}=\mathbf{x}_{i}^{\top} \boldsymbol{\beta}+f_{1}\left(t_{i 1}\right)+\ldots+f_{r}\left(t_{i r}\right)+\epsilon_{i}$, temos os modelos lineares parciais aditivos, em que as variáveis explicativas $t_{i l}$ contribuem de maneira não paramétrica para os modelos.

O objetivo desta Dissertação de Mestrado é descrever o método de suavização por meio de Psplines (Eilers e Marx, 1996) no contexto da família exponencial uniparamétrica, aplicando-o em duas classes de modelos: os modelos lineares parciais generalizados e os modelos lineares parciais aditivos generalizados, sendo que o primeiro é um caso particular do segundo. Supondo o modelo (1.1) e fazendo um paralelo com a função objetivo (1.2), o interesse agora é minimizar a função

$$
\sum_{i=1}^{n}\left\{y_{i}-f\left(x_{i}\right)\right\}^{2}+\frac{\lambda}{2} \gamma^{\top} \mathbf{P}_{d} \boldsymbol{\gamma},
$$

em que $f(x)$ pertence à classe dos B-splines (De Boor, 1978), $\gamma=\left(\gamma_{1}, \ldots, \gamma_{q}\right)^{\top}$ contém os parâmetros do B-spline selecionado, $\mathbf{P}_{d}$ funciona como um tipo de operador de diferenças entre os parâmetros em $\gamma$ e $\lambda>0$ é o parâmetro de suavização.

A vantagem de $f(x)$ pertencer à classe dos B-splines é a possibilidade de controlar por meio do grau do B-spline aspectos locais da relação funcional, bem como o número de nós e, consequentemente, o número de parâmetros a serem estimados. Por meio do operador de diferenças $\mathbf{P}_{d}$ é possível controlar a dependência entre intervalos adjacentes (no domínio de $x$ ) na estimação de $f(x)$.

Neste capítulo são apresentados a seguir três exemplos ilustrativos que podem ser analisados com modelagem semiparamétrica. No Capítulo 2 introduzimos os modelos lineares parciais generalizados (MLPGs) com suavização por meio de P-splines, discutimos alguns tipos de suavização, derivamos o processo iterativo para a estimação dos parâmetros e apresentamos alguns procedimentos usuais de seleção de modelos, inferência e diagnóstico. Estendemos os resultados do Capítulo 2 para a classe de modelos lineares parciais aditivos generalizados (MLPAGs) apresentados no Capítulo 3. 
Tabela 1.1: Ocorrência do início da menstruação nas garotas de Varsóvia.

\begin{tabular}{rrrrrr}
\hline Idade Média & Menstruadas & Entrevistadas & Idade Média & Menstruadas & Entrevistadas \\
\hline 9,21 & 0 & 376 & 13,33 & 67 & 106 \\
10,21 & 0 & 200 & 13,58 & 81 & 105 \\
10,58 & 0 & 93 & 13,83 & 88 & 117 \\
10,83 & 2 & 120 & 14,08 & 79 & 98 \\
11,08 & 2 & 90 & 14,33 & 90 & 97 \\
11,33 & 5 & 88 & 14,58 & 113 & 120 \\
11,58 & 10 & 105 & 14,83 & 95 & 102 \\
11,83 & 17 & 111 & 15,08 & 117 & 122 \\
12,08 & 16 & 100 & 15,33 & 107 & 111 \\
12,33 & 29 & 93 & 15,58 & 92 & 94 \\
12,58 & 39 & 100 & 15,83 & 112 & 114 \\
12,83 & 51 & 108 & 17,53 & 1049 & 1049 \\
13,08 & 47 & 99 & & & \\
\hline
\end{tabular}

Os exemplos de motivação apresentados neste capítulo são reanalisados sob o contexto semiparamétrico no Capítulo 4 por meio da biblioteca gamlss (Ribgy e Stasinopoulos, 2005) no software R. Considerações finais são apresentadas no Capítulo 5, enquanto que as derivações das funções escore e matrizes de informação de Fisher para os MLPGs e MLPAGs são apresentados, respectivamente, nos Apêndices A e B. No Apêndice C apresentamos a derivação do suavizador para as duas classes de modelos.

\subsection{Exemplos de motivação}

A seguir apresentamos três exemplos motivacionais que serão reanalisados no Capítulo 4 com a metodologia desenvolvida nesta dissertação.

\subsubsection{Garotas de Varsóvia}

Iniciamos com o exemplo das Garotas de Varsóvia descrito em Paula (2013). O objetivo do estudo realizado por Milicer e Szczotka (1965) é investigar a idade do início da menstruação. Sendo assim, foram coletadas aleatoriamente informações de 3918 garotas de diferentes escolas localizadas na cidade de Varsóvia, por meio de um questionário que perguntava a idade de cada uma das meninas e se elas já estavam menstruando ou não. Dessas garotas, 2080 tinham idade entre 11 e 16 anos. Para 25 médias de idades, as ocorrências do início da menstruação nas adolescentes foram observadas. A Tabela 1.1 descreve os dados resumidos.

O gráfico do logito da proporção de garotas com a mentruação já iniciada (Menstruadas/Entrevistadas) contra a idade média do grupo é apresentado na Figura 1.1. Para fins de visualização trocamos 0 por 0,5 e 1049 por 1048,5 .

Como era esperado, notamos que a proporção de garotas com menstruação já iniciada aumenta com a idade, porém de forma não linear na escala logito.

Devido ao comportamento não linear da proporção de garotas que já menstruavam conforme a idade, verificado na análise descritiva dos dados, vamos propor um modelo em que a variável idade 


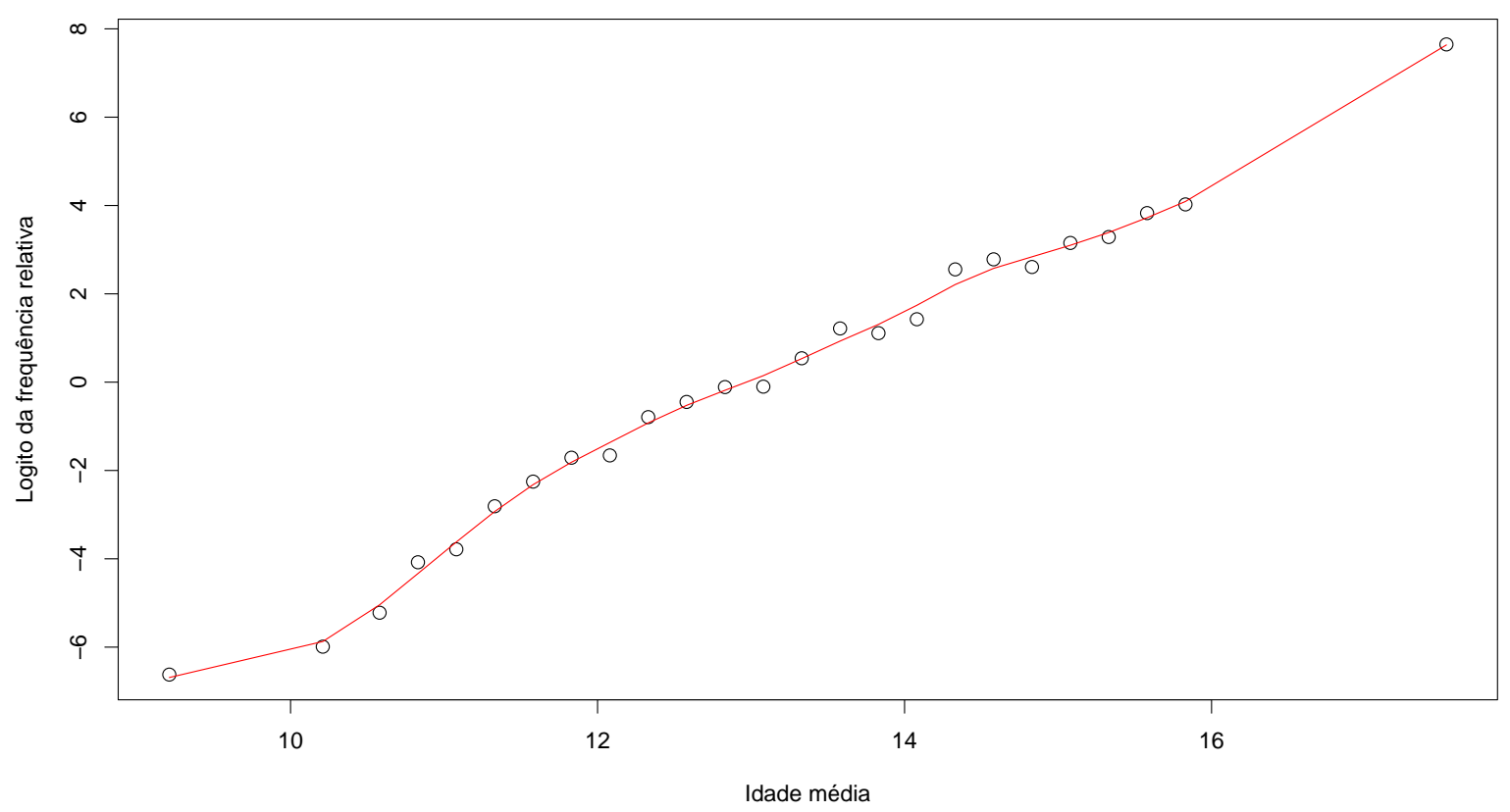

Figura 1.1: Gráfico do logito da frequência relativa de ocorrência de Menarca versus idade média para os dados de Garotas de Varsóvia, ajustados pela tendência.

é ajustada de forma não paramétrica. Assim, denotando por $y_{i}$ o número total de sucessos para a $i$-ésima idade média, ou seja, o número total de garotas que já menstruaram com idade média $i$, propomos o seguinte modelo:

$$
\begin{gathered}
y_{i} \mid i d a d e_{i} \stackrel{i n d}{\sim} B\left(m_{i}, \pi_{i}\right) \text { com } \\
\log \left\{\frac{\pi_{i}}{1-\pi_{i}}\right\}=\alpha+f\left(\text { idade }_{i}\right),
\end{gathered}
$$

em que $\pi_{i}$ denota a probabilidade de uma garota com idade na $i$-ésima classe de idade média já ter iniciado a menstruação, $m_{i}$ é o número de garotas entrevistadas na $i$-ésima classe de idade média, com $i=1, \ldots, 25$, e $f($.$) é uma função não paramétrica.$

O ajuste desse modelo será discutido no Capítulo 4.

\subsubsection{Clientes de uma loja}

O segundo exemplo que utilizaremos neste trabalho refere-se à dados de um estudo sobre o perfil de clientes de uma determinada loja, obtidos em Neter et al. (1996). Foram coletadas informações de clientes de 110 áreas diferentes de uma cidade que frequentavam certa loja. O objetivo do estudo é relacionar o número esperado de clientes de cada área segundo algumas características dessa área. As variáveis observadas em cada área são as seguintes:

- nclientes: Número de clientes,

- domic: Número de domicílios,

- renda: Renda média anual (em USD), 
- idade: Idade média dos domicílios (em anos),

- dist1: Distância ao concorrente mais próximo (em milhas) e

- dist2: Distância à loja (em milhas).

Vamos inicialmente descrever cada variável observada por meio de boxplots robustos (consultar Hubert e Vandervieren, 2008) apresentados nas Figuras 1.2 e 1.3.

Nota-se que apenas para as variáveis número de clientes e renda os gráficos destacam alguns pontos aberrantes. O boxplot robusto indica que não é comum que poucos clientes (menos de cinco) de uma mesma área frequentem a loja. O mesmo acontece para a variável renda, em que, no boxplot robusto, clientes que possuem renda mais baixa e frequentam a loja são considerados pontos aberrantes. Com relação à variável distância à loja, vemos que não há nenhum ponto aberrante no boxplot. Esses são os casos que apresentam uma assimetria, possível de notar observando a mediana dos boxplots robustos. As demais variáveis estudadas têm um comportamento simétrico e não indicam nenhum ponto aberrante nas observações.

Além dos boxplots, vamos analisar a dispersão dos dados da variável resposta número de clientes na escala log contra as variáveis explicativas. Para fins de visualização, nos casos em que não haviam clientes de uma determinada área, trocamos 0 por 0,5. Os gráficos são apresentados na Figura 1.4.

Vemos que o número de clientes aumenta de forma não linear quando a distância ao concorrente mais próximo fica maior. Além disso, nota-se o número de clientes aumenta quando há mais domícilios em uma determinada área e diminui quando a distância do domícilio à loja fica maior, sendo que essas duas variáveis parecem ter um leve comportamento não linear. Por fim, vemos que o número de clientes fica praticamente constante quando considerada a variável idade e que aumenta lentamente à medida que a renda aumenta.

As correlações entre as variáveis são apresentadas na Figura 1.5. Nota-se que a maior correlação é entre o número de clientes que frequentam a loja e as variáveis distância ao concorrente mais próximo e distância à loja.

A partir da análise descritiva dos dados, vamos propor um modelo em que as variáveis domicílio (domic), distância ao concorrente mais próximo (dist1) e distância à loja (dist2) serão ajustadas de forma não paramétrica (de modo que verificaremos por meio do ajuste do modelo se essas variáveis realmente têm comportamento não linear) e as variáveis idade e renda de forma paramétrica. Sendo assim, definindo $y_{i}$ como sendo o número de clientes da $i$-ésima área que visitaram a loja num determinado período, vamos supor que essa variável segue uma distribuição de Poisson tal que:

$$
\begin{gathered}
y_{i} \mid \mathbf{x}_{i} \stackrel{i n d}{\sim} P\left(\mu_{i}\right) \text { com } \\
\log \left(\mu_{i}\right)=\beta_{0}+\beta_{1} \text { renda }_{i}+\beta_{2} i \operatorname{dade}_{i}+f_{1}\left(\text { domic }_{i}\right)+f_{2}\left(\text { dist }_{i}\right)+f_{3}\left(\operatorname{dist}_{i}\right),
\end{gathered}
$$

em que $\mathbf{x}_{i}=\left(1, \text { renda }_{i}, \text { idade }_{i}, \text { domic }_{i} \text {, dist } 1_{i}, \text { dist }_{i}\right)^{\top}, \mu_{i}$ denota o número esperado de clientes da $i$-ésima área dados os valores das variáveis explicativas, para $i=1, \ldots, 110$, enquanto $f_{1}(),. f_{2}($.$) e$ $f_{3}($.$) são funções não paramétricas.$

\subsubsection{Valor de aluguel em Munique}

Nesta seção apresentamos os dados sobre o valor do aluguel na cidade de Munique, disponível no pacote gamlss.data como rent99. 

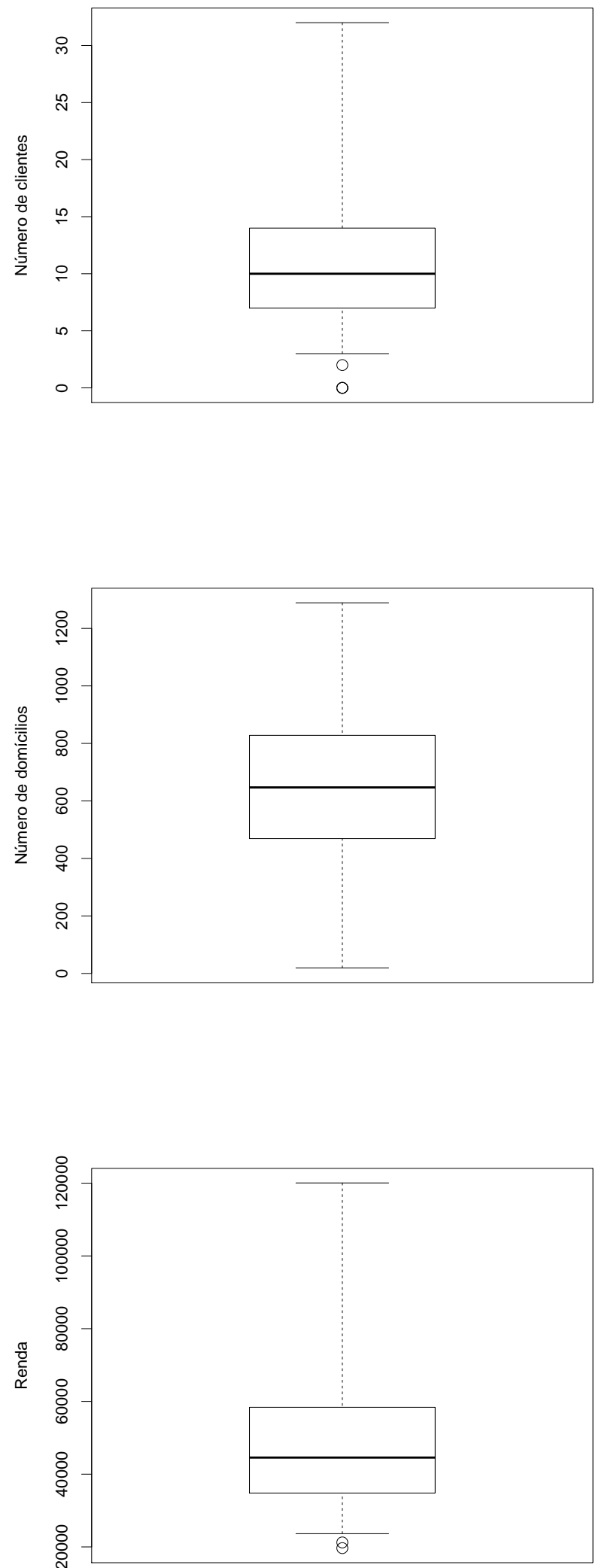

Figura 1.2: Gráficos boxplots robustos das variáveis número de clientes, número de domicilios e renda média referentes aos dados Clientes de uma Loja. 

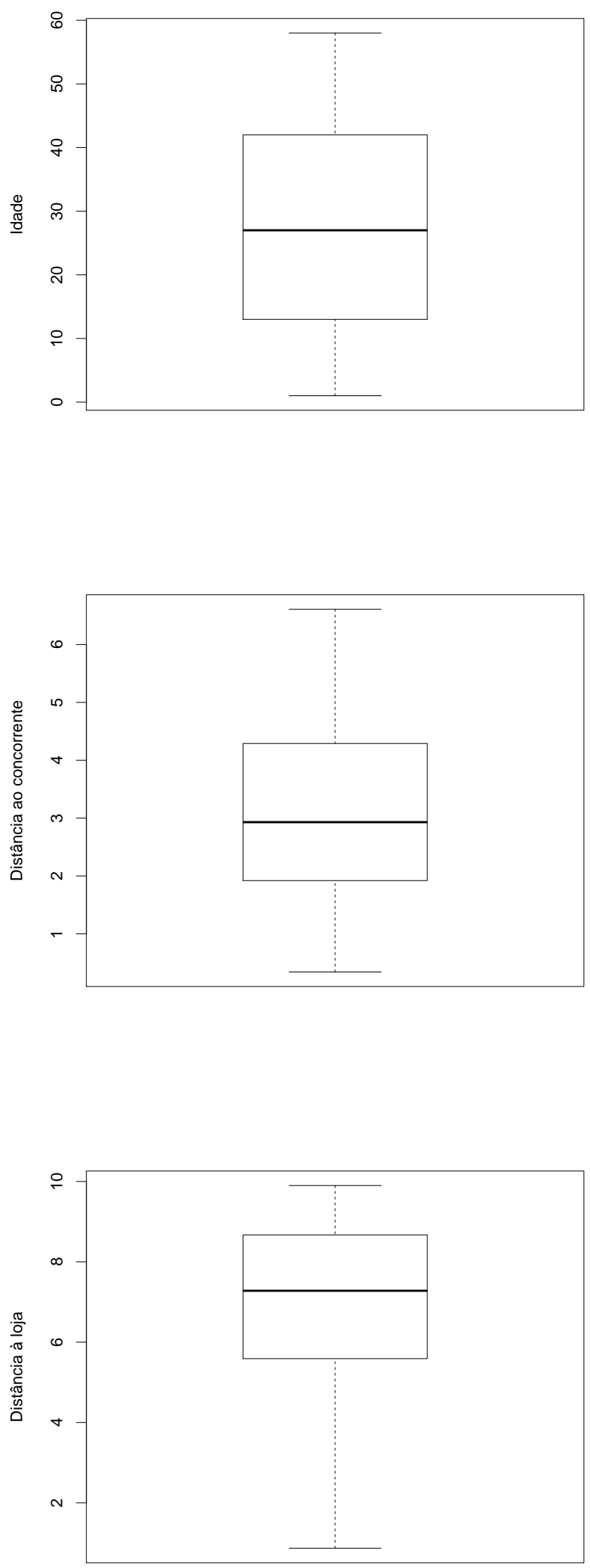

Figura 1.3: Gráficos boxplots robustos das variáveis idade, distância ao concorrente mais próximo e distância à loja referentes aos dados Clientes de uma Loja. 



Figura 1.4: Gráficos de dispersão do log da variável número de clientes contra as variáveis explicativas referentes aos dados Clientes de uma Loja, ajustados pelas respectivas tendências. 


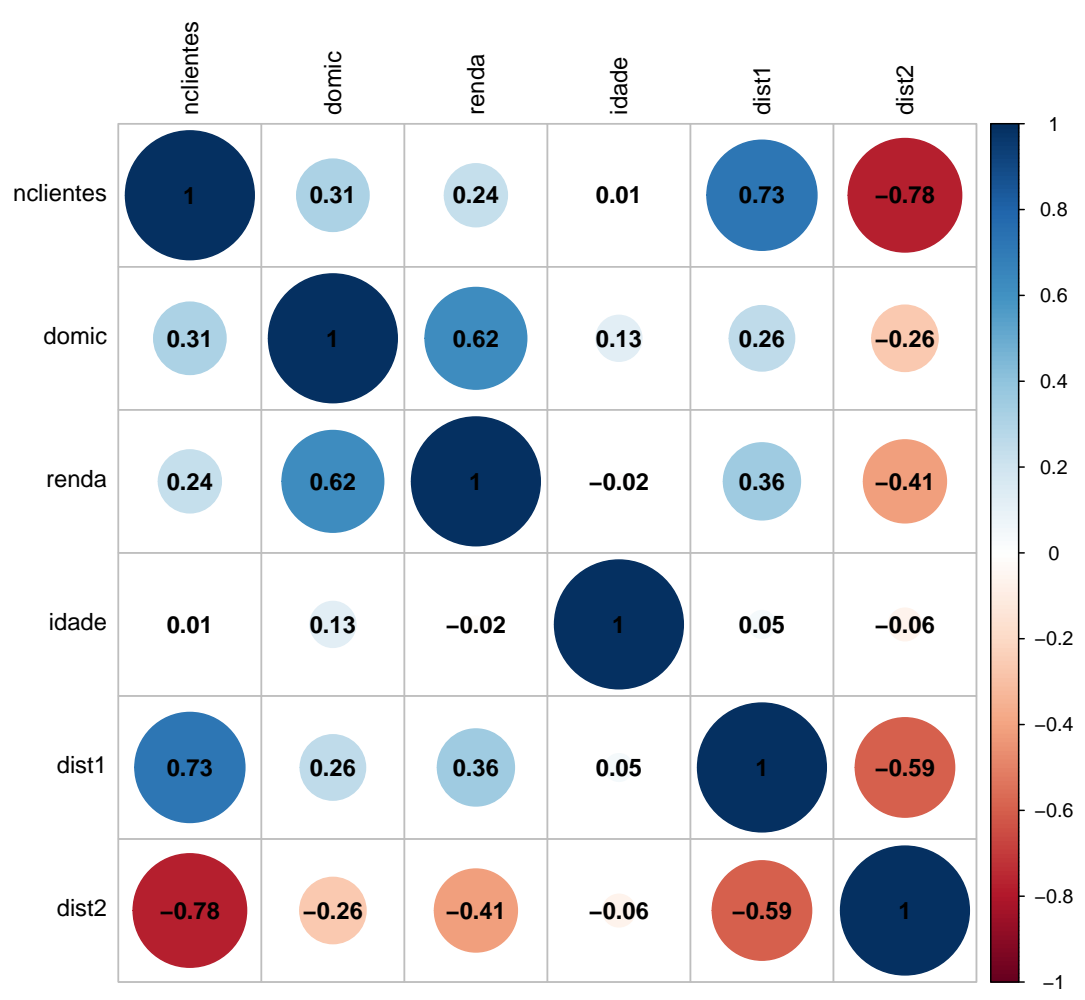

Figura 1.5: Gráfico de correlação entre as variáveis referentes aos dados Clientes de uma Loja.

Foram coletadas informações de 3082 apartamentos a fim de entender as características que influenciam o valor do aluguel cobrado mensalmente em Munique. Sendo assim, vamos analisar as sete variáveis apresentadas a seguir:

- Valor mensal do aluguel (em euros),

- area: Tamanho do apartamento (em metros quadrados),

- ano: Ano de construção (período),

- loc: Qualidade da localização (1: Média, 2: Boa e 3: Excelente),

- banh: Qualidade do banheiro (0: Padrão e 1: Premium),

- coz: Qualidade da cozinha (0: Padrão e 1: Premium) e

- aquec: Aquecimento central (0: Sem aquecimento e 1: Com aquecimento).

Dessa forma, estudaremos o valor do aluguel cobrado mensalmente segundo as variáveis explicativas descritas acima.

Preliminarmente, vamos analisar o comportamento do valor do aluguel. A Figura 1.6 indica que a variável resposta valor do aluguel segue aproximadamente uma distribuição assimétrica à direita, sugerindo, por exemplo, uma distribuição gama.

Na Figura 1.7 são apresentados os gráficos do log do valor do aluguel contra cada variável explicativa quantitativa. Analisando inicialmente o gráfico do log do valor do aluguel contra a 


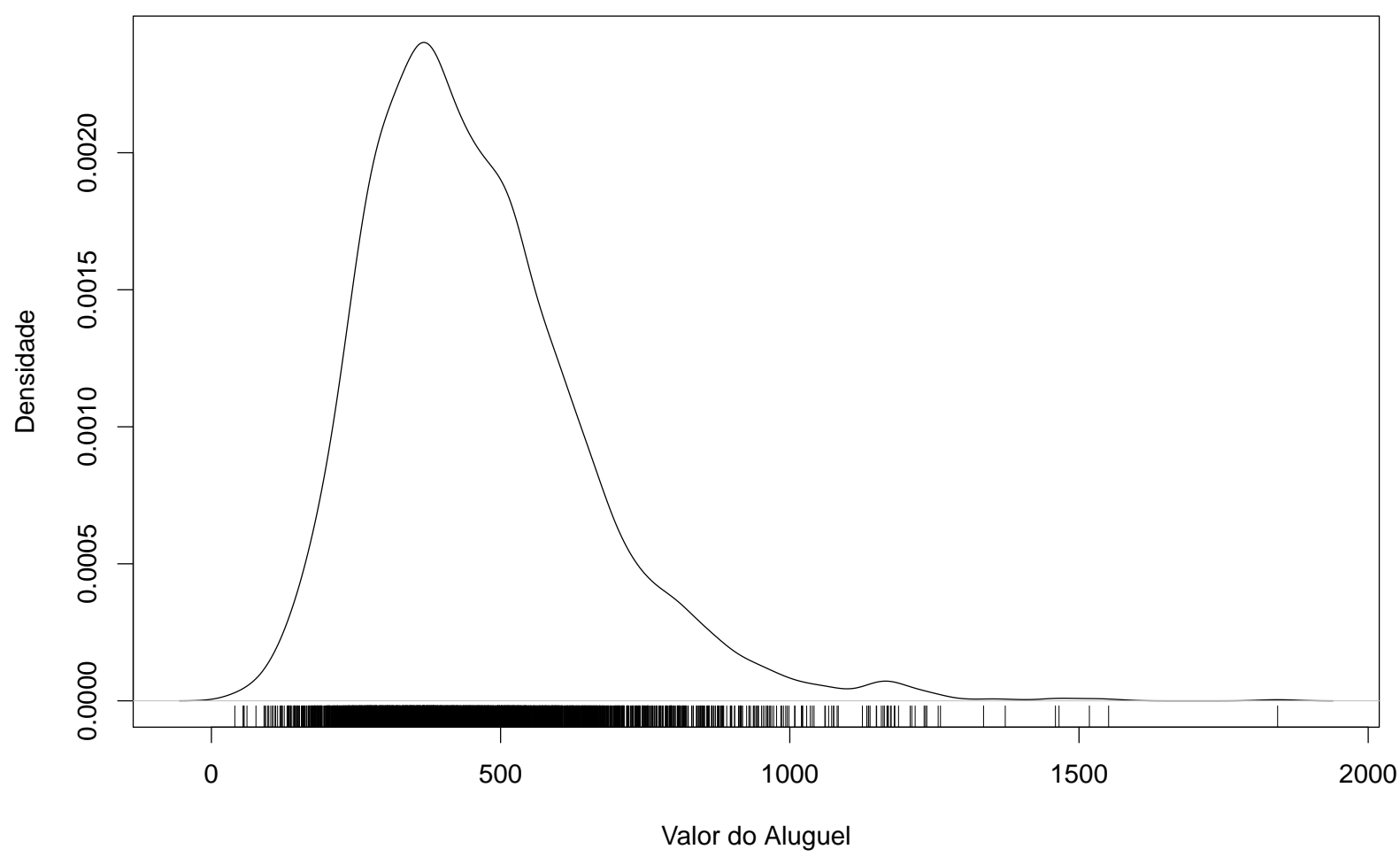

Figura 1.6: Gráfico da densidade do valor do aluguel para os dados Valor de Aluguel em Munique.

área do apartamento, é possível observar que há uma tendência positiva, ou seja, quanto maior o apartamento, mais caro é o aluguel cobrado, sendo que o comportamento do valor do aluguel aparenta ser não linear neste caso. Com relação à variável explicativa ano de construção, parece existir uma tendência não linear de aumento do valor do aluguel para apartamentos construídos após 1960, de forma que, se o apartamento foi construído antes desse ano, o valor cobrado pelo aluguel varia pouco. Na Figura 1.8 são apresentadas as correlações entre essas três variáveis. Vemos que a maior correlação é entre o valor do aluguel e o tamanho do apartamento. Já, a variável ano de construção tem uma correlação baixa com o valor do aluguel, o que pode ser explicado pela pouca variação do aluguel cobrado em apartamentos construídos antes de 1960.

Para as variáveis explicativas qualitativas, foram construídos boxplots robustos apresentados nas Figuras 1.9 e 1.10 .

Analisando os boxplots dessas variáveis, nota-se que a mediana do valor do aluguel aumenta quando a localização do apartamento é classificada como excelente, aumenta quando as instalações da cozinha e do banheiro são premium e quando há aquecimento central no apartamento. Além disso, é possível observar pelos gráficos a assimetria presente nos dados.

Com base na análise descritiva, percebemos que as varáveis área e ano de construção aparentam ter comportamento não linear no log do valor do aluguel. Dessa forma, vamos introduzir essas variáveis de forma não paramétrica no modelo.

Vamos propor inicialmente o seguinte modelo:

$$
y_{i} \mid \mathbf{x}_{i} \stackrel{i n d}{\sim} G\left(\mu_{i}, \sigma\right) \text { com }
$$



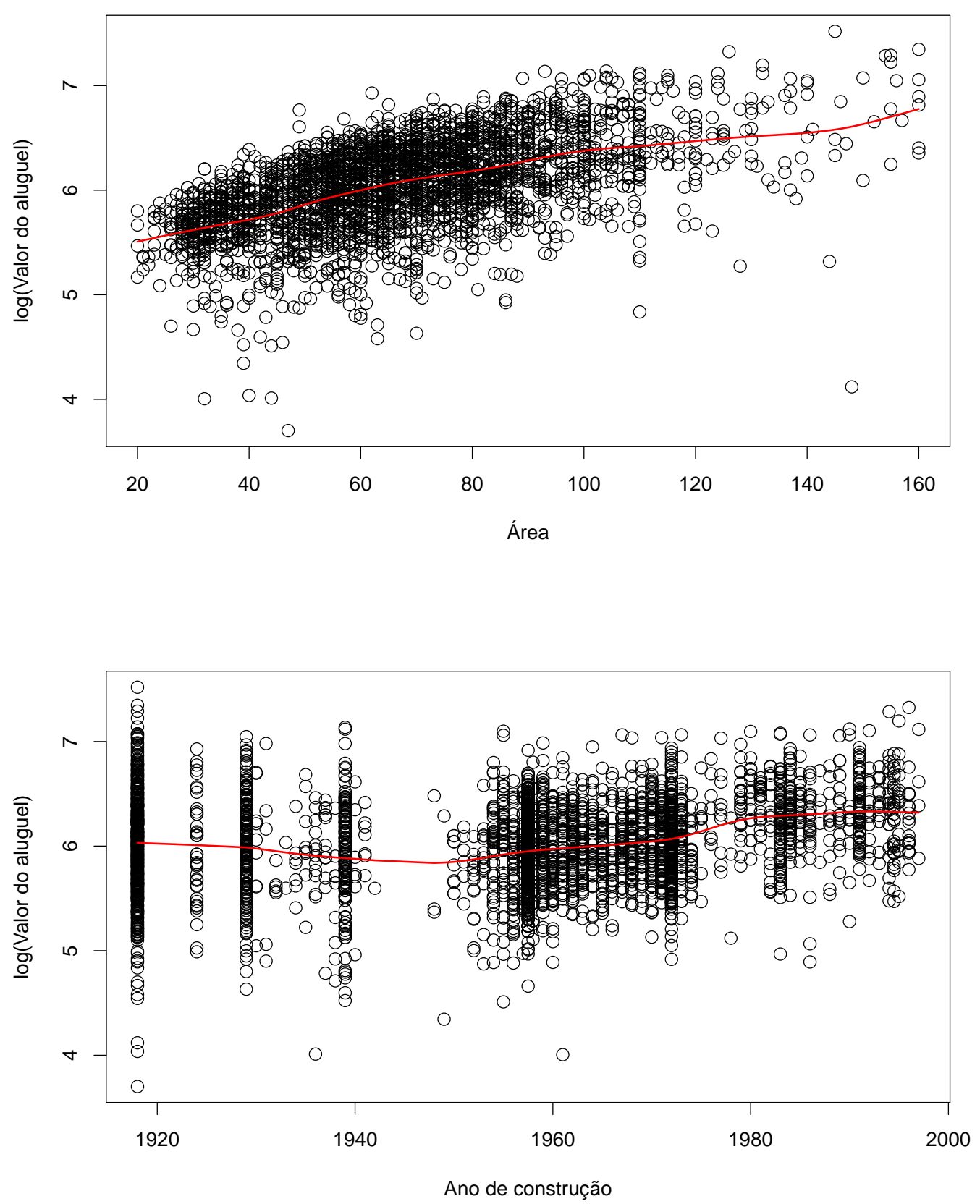

Figura 1.7: Gráficos de dispersão do log do valor do aluguel contra variáveis explicativas contínuas para os dados de Valor do Aluguel, ajustados pelas respectivas tendências. 




Figura 1.8: Gráfico de correlação entre as variáveis quantitativas referentes aos dados Valor do Aluguel.

$$
\log \left(\mu_{i}\right)=\beta_{0}+\beta_{11} l_{\text {loc }}{ }_{1 i}+\beta_{12} l_{o c} c_{2 i}+\beta_{2} \text { banh }_{i}+\beta_{3} \text { coz }_{i}+\beta_{4} \text { aquec }_{i}+f_{1}\left(\text { area }_{i}\right)+f_{2}\left(\text { ano }_{i}\right),
$$

em que $\mathbf{x}_{i}=\left(1, l_{l o c}{ }_{1 i}, l_{l o c} i, \text { banh }_{i}, \text { coz }_{i}, \text { aquec }_{i}, \text { area }_{i}, a n o_{i}\right)^{\top}, l o c_{1}, l o c_{2}$, banh, coz e aquec são variáveis binárias referentes, respectivamente, aos efeitos qualidade da localização boa, qualidade da localização excelente, qualidade do banheiro premium, qualidade da cozinha premium e presença de aquecimento central, enquanto $f_{1}($.$) e f_{2}($.$) são funções não paramétricas e \sigma$ denota o coeficiente de variação de $y_{i}$, para $i=1, \ldots, 3082$. 



Figura 1.9: Gráficos boxplots robustos do log do valor do aluguel segundo os níveis das variáveis explicativas qualitativas localização e instalações do banheiro para os dados de Valor do Aluguel. 

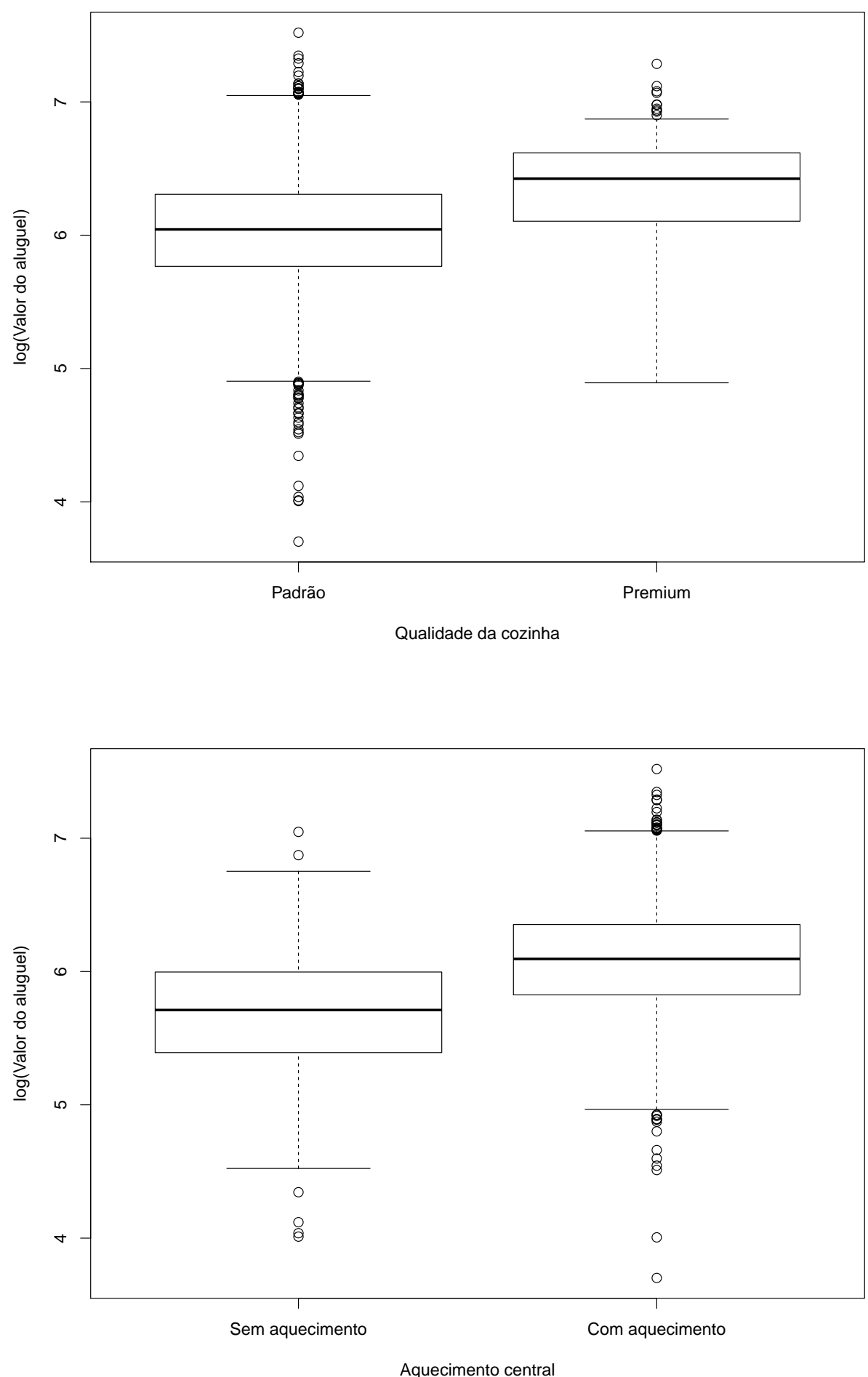

Figura 1.10: Gráficos boxplots robustos do log do valor do aluguel segundo os níveis das variáveis explicativas qualitativas qualidade da cozinha e aquecimento central para os dados de Valor do Aluguel. 


\section{Capítulo 2}

\section{Modelos lineares parciais generalizados}

Os modelos lineares parciais generalizados consideram, além das funções paramétricas adotadas nos modelos lineares generalizados, uma função não paramétrica para descrever a relação entre a média da variável resposta e alguma variável explicativa com comportamento não linear. Sendo assim, essa categoria de modelos nada mais é do que uma extensão dos MLGs.

Dentre os principais trabalhos relacionados aos modelos lineares parciais estão Green e Yandell (1985) que descrevem os modelos lineares generalizados semiparamétricos utilizando splines cúbicos, apresentam as estimativas de máxima verossimilhança penalizada e métodos para estimar o parâmetro de suavização. Uma referência clássica sobre esses modelos é Green e Silverman (1994), que apresentam um estudo detalhado sobre a regressão não paramétrica, splines e modelos lineares generalizados. Fahrmeir e Tutz (2001) descrevem a teoria de modelos semiparamétricos e não paramétricos e apresentam diversas aplicações, com alguns exemplos utilizados no livro estando também disponíveis no pacote Fahrmeir, no software R.

Sendo assim, neste capítulo apresentaremos inicialmente uma breve revisão dos modelos lineares generalizados e da família exponencial, e posteriormente a teoria dos modelos lineares parciais generalizados utilizando P-splines. Assim, na Seção 2.2 especificamos o modelo a ser estudado e na Seção 2.3 apresentamos a teoria do método de suavização P-splines, que é uma combinação entre os B-splines e uma penalização de contrastes de parâmetros. Já na Seção 2.4 definimos a função de penalização dos P-splines que será utilizada no ajuste do modelo neste trabalho, além de introduzir brevemente outra forma de penalização aplicada aos splines cúbicos. Nessa mesma seção são obtidas a função de verossimilhança penalizada, as funções escore penalizadas e a matriz de informação de Fisher penalizada. Na Seção 2.5 são utilizados os resultados obtidos na seção anterior para a estimação dos parâmetros do modelo. Além disso, são definidos os graus de liberdade efetivos do modelo e apresentados métodos para estimar o parâmetro de suavização. Na Seção 2.6 são apresentados alguns resultados assintóticos e teste de hipóteses. Por fim, na Seção 2.7 discutimos análise de resíduos.

\subsection{Revisão dos modelos lineares generalizados}

Antes de apresentarmos a teoria do modelos lineares parciais generalizados, vamos fazer um breve resumo sobre os MLGs e a família exponencial.

A variável resposta dos modelos lineares generalizados pode assumir uma variedade de distribuições desde que essas pertençam à família exponencial. 
Tabela 2.1: Principais distribuições que pertencem à família exponencial.

\begin{tabular}{lrrrr}
\hline Distribuição & $\boldsymbol{\theta}$ & $\boldsymbol{b}(\boldsymbol{\theta})$ & $\boldsymbol{\phi}$ & $\boldsymbol{V}(\boldsymbol{\mu})$ \\
\hline Normal & $\mu$ & $\theta^{2} / 2$ & $\sigma^{-2}$ & 1 \\
Poisson & $\log (\mu)$ & $e^{\theta}$ & 1 & $\mu$ \\
Binomial & $\log \{\mu /(1-\mu)\}$ & $\log \left(1+e^{\theta}\right)$ & $n$ & $\mu(1-\mu)$ \\
Gama & $-1 / \mu$ & $-\log (-\theta)$ & $1 /\left(\sigma^{1}\right)^{2}$ & $\mu^{2}$ \\
N. Inversa & $-1 /\left(2 \mu^{2}\right)$ & $-(-2 \theta)^{1 / 2}$ & $\phi$ & $\mu^{3}$ \\
\hline
\end{tabular}

${ }^{1} \mathrm{Na}$ distribuição gama, $\sigma$ denota o coeficiente de variação.

Diz-se que as variáveis aleatórias $y_{1}, y_{2}, \ldots, y_{n}$ têm distribuição pertencente à família exponencial (FE) quando as suas funções densidade de probabilidade (f.d.p.) ou funções de probabilidade podem ser escritas da seguinte maneira:

$$
f\left(y_{i} ; \theta_{i}, \phi\right)=\exp \left[\phi\left\{y_{i} \theta_{i}-b\left(\theta_{i}\right)\right\}+c\left(y_{i}, \phi\right)\right],
$$

em que $\theta_{i}$ é o parâmetro canônico de localização, $\phi$ é o parâmetro de precisão e $b($.$) e c($.$) são$ funções contínuas e diferenciáveis nos argumentos, para $i=1, \ldots, n$.

Considerando as seguintes condições de regularidade:

$$
E\left\{\frac{\partial L\left(\theta_{i} ; \phi\right)}{\partial \theta_{i}}\right\}=0 \quad \text { e } \quad E\left[\frac{\partial^{2} L\left(\theta_{i} ; \phi\right)}{\partial \theta_{i}{ }^{2}}\right]=-E\left[\left\{\frac{\partial L\left(\theta_{i} ; \phi\right)}{\partial \theta_{i}}\right\}^{2}\right]
$$

em que $L\left(\theta_{i}, \phi\right)=\log \left\{f\left(y_{i} ; \theta_{i} ; \phi\right)\right\}=\phi\left\{y_{i} \theta_{i}-b\left(\theta_{i}\right)\right\}+c\left(y_{i}, \phi\right)$, podemos mostrar que $\mu_{i}=E\left(y_{i} \mid x_{i}\right)=$ $b^{\prime}\left(\theta_{i}\right)$ e $\operatorname{Var}\left(y_{i}\right)=\phi^{-1} V\left(\mu_{i}\right)$, sendo $V_{i}=V\left(\mu_{i}\right)=b^{\prime \prime}\left(\theta_{i}\right)=\frac{d \mu_{i}}{d \theta_{i}}$ a função de variância.

Na Tabela 2.1 é apresentado um resumo com as principais distribuições que pertencem à família exponencial e suas respectivas quantidades.

Uma vez definida a família exponencial, podemos descrever os modelos lineares generalizados. Sejam $y_{1}, y_{2}, \ldots, y_{n}$ variáveis aleatórias independentes. Os modelos lineares generalizados são definidos por

$$
\begin{gathered}
\text { - } y_{i} \mid x_{i} \stackrel{i n d}{\sim} \mathrm{FE}\left(\mu_{i}, \phi\right), \\
\text { - } g\left(\mu_{i}\right)=\eta_{i} \mathrm{e} \\
\text { - } \eta_{i}=\mathbf{x}_{i}^{\top} \boldsymbol{\beta},
\end{gathered}
$$

em que $\eta_{i}$ é o preditor linear, $\boldsymbol{\beta}=\left(\beta_{1}, \ldots, \beta_{p}\right)^{\top}$ é o vetor de parâmetros a serem estimados, com $p<n, \mathbf{x}_{i}=\left(x_{i 1}, \ldots, x_{i p}\right)^{\top}$ são os valores das variáveis explicativas e $g($.) é uma função monótona e diferenciável, chamada de função de ligação.

Quando o preditor linear coincide com o parâmetro canônico $\theta$, isto é, $\theta_{i}=\eta_{i}=\sum_{j=1}^{p} x_{i j} \beta_{j}$, a função de ligação correspondente é chamada de ligação canônica.

Tomando dois exemplos, sabemos que para a distribuição normal $\theta=\mu$ e que para a distribuição binomial $\theta=\log \left\{\frac{\mu}{1-\mu}\right\}=\operatorname{logito}(\mu)$ com $0<\mu<1$. Dessa forma, a ligação canônica para essas duas distribuições são $\mu=\eta$ e $\log \left\{\frac{\mu}{1-\mu}\right\}=\eta$, respectivamente.

Na Tabela 2.2 são apresentadas as ligações canônicas mais comuns. 
Tabela 2.2: Ligações canônicas mais comuns nos MLGs.

\begin{tabular}{lrrrrr}
\hline Distribuição & Normal & Poisson & Binomial & Gama & N.Inversa \\
\hline Ligação & $\mu=\eta$ & $\log (\mu)=\eta$ & $\log \left\{\frac{\mu}{1-\mu}\right\}=\eta$ & $\mu=\frac{1}{\eta}$ & $\mu=\frac{1}{\eta^{2}}$ \\
\hline
\end{tabular}

A ligação $\mu=\eta$ é chamada de identidade, a $\log (\mu)=\eta$ de logarítmica, a ligação $\mu=\frac{1}{\eta}$ é chamada de recíproca, a $\mu=\frac{1}{\eta^{2}}$ de recíproca ao quadrado e a ligação $\log \left\{\frac{\mu}{1-\mu}\right\}$ de logito.

Pode-se utilizar outros tipos de ligações, chamadas de não canônicas. A seguir serão apresentadas as principais.

A ligação probito é definida como

$$
\Phi^{-1}(\mu)=\eta
$$

em que $\mu$ é a proporção de sucessos da distribuição binomial e $\Phi($.$) é a função de distribuição$ acumulada da normal padrão.

Uma outra ligação utilizada é a ligação de Box e Cox (1964), muito importante para ser aplicada quando as observações são positivas, sendo definida por

$$
\eta=\left\{\begin{aligned}
\frac{\mu^{\lambda}-1}{\lambda}, & \text { se } \lambda \neq 0 \\
\log (\mu), & \text { se } \lambda=0
\end{aligned}\right.
$$

para $\mu>0$ e $y>0$.

A ligação de Aranda-Ordaz (1981) foi proposta para proporções. Sua definição é apresentada abaixo

$$
\eta=\log \left\{\frac{(1-\mu)^{-\alpha}-1}{\alpha}\right\}
$$

em que $0<\mu<1$ e $\alpha>0$ é uma constante desconhecida. Quando $\alpha=1$ obtemos a ligação logito $\eta=\log \left\{\frac{\mu}{1-\mu}\right\}$. Agora, se $\alpha \rightarrow 0$ obtemos uma outra ligação chamada de complemento log-log, definida por

$$
\eta=\log \{-\log (1-\mu)\}
$$

Para mais detalhes sobre os MLGs, ver McCullagh e Nelder (1989) e Paula (2013).

\subsection{Especificação do modelo}

Os modelos lineares parciais generalizados (MLPGs) permitem a inserção de uma função não paramétrica no preditor linear dos modelos lineares generalizados, flexibilizando a relação entre as variáveis explicativas e a média da variável resposta.

Os modelos lineares parciais generalizados são definidos por

$$
\begin{gathered}
y_{i} \mid\left(\mathbf{x}_{i}, t_{i}\right) \stackrel{i n d}{\sim} \mathrm{FE}\left(\mu_{i}, \phi\right) \mathrm{com} \\
g\left(\mu_{i}\right)=\eta_{i}=\mathbf{x}_{i}^{\top} \boldsymbol{\beta}+f\left(t_{i}\right),
\end{gathered}
$$


em que $\eta_{i}$ é o preditor linear, $g($.$) é a função de ligação, \boldsymbol{\beta}=\left(\beta_{1}, \ldots, \beta_{p}\right)^{\top}$ é o vetor de coeficientes a serem estimados, $\phi$ é o parâmetro de precisão, $\mathbf{x}_{i}=\left(x_{i 1}, \ldots, x_{i p}\right)^{\top}$ contém os valores das variáveis explicativas tratadas de forma paramétrica, $f($.$) é uma função contínua e diferenciável, t_{i}$ denota o valor de uma covariável contínua que contribui de maneira não linear, para $i=1, \ldots, n$, e FE denota a família exponencial de distribuições.

Como vimos anteriormente, a função não paramétrica pode assumir diversas formas. Adotaremos como função não paramétrica o método de suavização P-splines, que permite um maior controle sobre a suavização obtida para o modelo. Sendo assim, iremos expressar $f\left(t_{i}\right)$ na seguinte forma:

$$
f\left(t_{i}\right)=\sum_{j=1}^{q} B_{j}^{(m)}\left(t_{i}\right) \gamma_{j},
$$

em que $q$ é a dimensão da base dos B-splines (De Boor, 1978), $k=q+m+1$ é o número de nós, $B_{j}^{(m)}\left(t_{i}\right)$ é o $j$-ésimo componente da base que é um B-spline de ordem $m+1$ no ponto $t_{i}$ e $\gamma_{j}$ são os coeficientes para serem estimados, com $j=1, \ldots, q$ e $m=0,1,2, \ldots$. Usualmente, adota-se $m=3$ (ordem $=4$ ), ajustando um B-spline cúbico em cada intervalo.

Podemos reescrever a parte sistemática (2.1) como

$$
g\left(\mu_{i}\right)=\eta_{i}=\mathbf{x}_{i}^{\top} \boldsymbol{\beta}+\mathbf{b}_{i}^{\top} \boldsymbol{\gamma},
$$

em que $\mathbf{b}_{i}=\left(B_{1}^{(m)}\left(t_{i}\right), \ldots, B_{q}^{(m)}\left(t_{i}\right)\right)^{\top}$ e $\gamma=\left(\gamma_{1}, \ldots, \gamma_{q}\right)^{\top}$.

A equação acima em forma matricial fica dada por

$$
\mathbf{g}(\boldsymbol{\mu})=\boldsymbol{\eta}=\mathbf{X} \boldsymbol{\beta}+\mathbf{B} \boldsymbol{\gamma},
$$

em que $\mathbf{X}$ é uma matriz de dimensão $n \times p$ formada pelos elementos $x_{i j}$ e $\mathbf{B}$ é uma matriz de dimensão $n \times q$ formada pelos componentes $B_{j}^{(m)}\left(t_{i}\right)$ da base de De Boor $(1978), i=1, \ldots, n$ e $j=1, \ldots, q$.

Definido o modelo que discutiremos neste capítulo, apresentamos na próxima seção a teoria do método de suavização P-splines, que nada mais é do que a aplicação de uma penalização de diferenças nos coeficientes dos B-splines.

\subsection{Método de suavização P-splines}

Como os P-splines são uma combinação entre o método de suavização B-splines e uma penalização de diferenças, torna-se necessário apresentar o conceito dos B-splines antes de definirmos os $\mathrm{P}$-splines.

Os splines básicos, mais conhecidos como B-splines, foram propostos por De Boor (1978). Esses splines são construídos a partir de pedaços de polinômios que se unem de forma suavizada em pontos chamados de nós, evitando que entre esses pontos ocorram mudanças abruptas na função.

Sendo assim, o domínio dos dados $\left[t_{1}, \ldots, t_{n}\right]$ é dividido em intervalos menores, delimitados pelos nós. Ao utilizar os B-splines, ajusta-se polinômios de baixo grau em cada intervalo definido, diferente do que é feito nos modelos polinomiais, que utilizam polinômios com alto grau para obter o ajuste. Desta forma, o uso da base de De Boor é favorável, já que exige pouco esforço computacional.

Além disso, a utilização dos B-splines é interessante porque as funções base são estritamente 
locais, de forma que cada função da base de De Boor assume valores diferentes de zero nos intervalos que abrangem $m+3$ nós adjacentes, em que $m+1$ é a ordem dos B-splines.

A escolha de quantos nós serão utilizados no ajuste é muito importante, pois essa escolha determinará a qualidade do ajuste do modelo. Além disso, a definição da posição dos nós também é um assunto relevante e pode ser feita de diversas formas. O primeiro fator a ser definido é se os nós serão equidistantes entre si ou não. O segundo fator é onde posicionar o primeiro nó, podendo estar no primeiro valor observado da variável que será ajustada ou onde ocorre o primeiro ponto de mudança. Yu e Ruppert (2002) propõem que os nós sejam equidistantes e posicionados nos quantis das variáveis preditoras. Já Vanegas e Paula (2016) definem o número de nós como $k=n^{\frac{1}{3}}+3$, sendo estes posicionados nos quantis $q\left(t, \frac{1}{k+1}\right), \ldots, q\left(t, \frac{k}{k+1}\right)$ de $t_{i}$. Para mais detalhes de como selecionar o número de nós e onde posicioná-los, consultar Ruppert (2002).

Os B-splines, $B_{j}^{(m)}(x)$, são definidos recursivamente como

$$
B_{j}^{(0)}(x)= \begin{cases}1, & \text { se } t_{j} \leq x \leq t_{j+1} \\ 0, & \text { caso contrário }\end{cases}
$$

$\mathrm{e}$

$$
B_{j}^{(m)}(x)=\frac{x-t_{j}}{t_{j+m}-t_{j}} B_{j}^{(m-1)}(x)+\frac{t_{j+m+1}-x}{t_{j+m+1}-t_{j+1}} B_{j+1}^{(m-1)}(x),
$$

em que $x \in[a, b], k$ é o número de nós sendo que $a<t_{1}, t_{2}, \ldots, t_{k}<b, m=1,2, \ldots$ é o grau dos B-splines, $k=q+m+1$ é o número de nós e $B_{j}^{(m)}(x)$ é o valor do $j$-ésimo B-spline de grau $m$ no ponto $x$ para uma malha de nós equidistantes, $j=1, \ldots, q$.

Para compreender melhor a maneira de computar $B_{j}^{(m)}(x)$ para $m \geq 0$, é utilizado o esquema de computação triangular, apresentado no Figura 2.1.

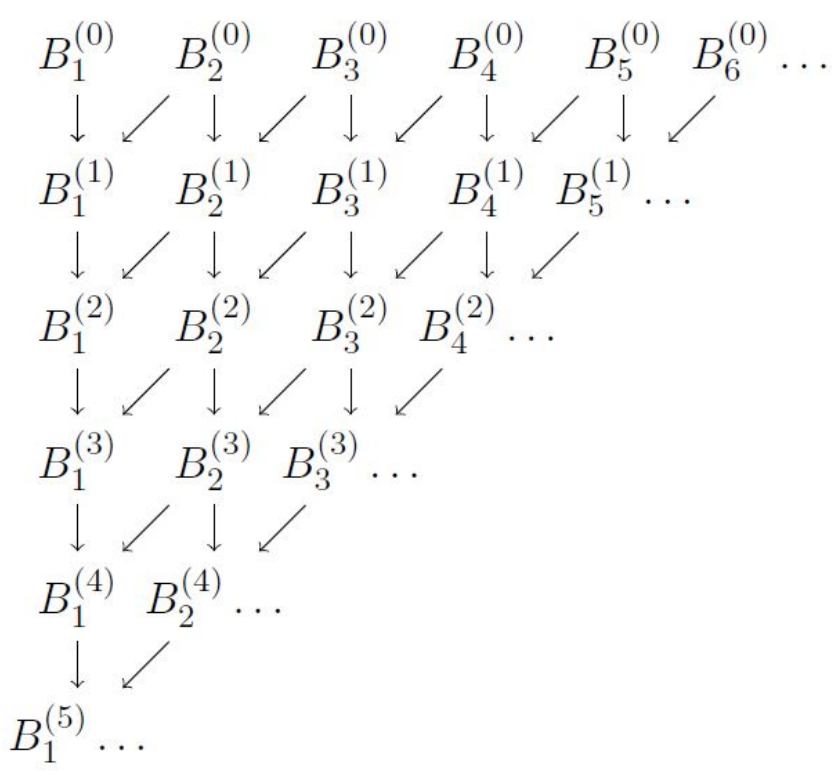

Figura 2.1: Diagrama do esquema de Computação Triangular dos B-splines.

Sendo assim, para computar $B_{j}^{(m)}(x)$, para $m>0$, são exigidos $B_{j}^{(m-1)}(x)$ e $B_{j+1}^{(m-1)}(x)$. Por exemplo, calculando $B_{1}^{(1)}(x)$, temos 


$$
B_{1}^{(1)}(x)=\frac{x-t_{1}}{t_{2}-t_{1}} B_{1}^{(0)}+\frac{t_{3}-x}{t_{3}-t_{2}} B_{2}^{(0)}(x) .
$$

A Figura 2.2 apresenta os gráficos de B-splines de grau 1 considerando nós em 0, 0,25, 0,5, 0,75 e 1. Nota-se que um B-spline de grau 1 é formado por dois pedaços lineares e três nós, sendo que os pedaços se encontram em um nó interior. No exemplo em questão, apresentado na Figura 2.2a, o primeiro pedaço é formado pelos nós 0 e 0,25 e o segundo pelos nós 0,25 e 0,5 . À esquerda do primeiro nó e à direita do último nó, o B-spline assume valor 0. Já, na Figura 2.2b podemos observar mais exemplos de B-splines de grau 1 formados pelos mesmos nós.

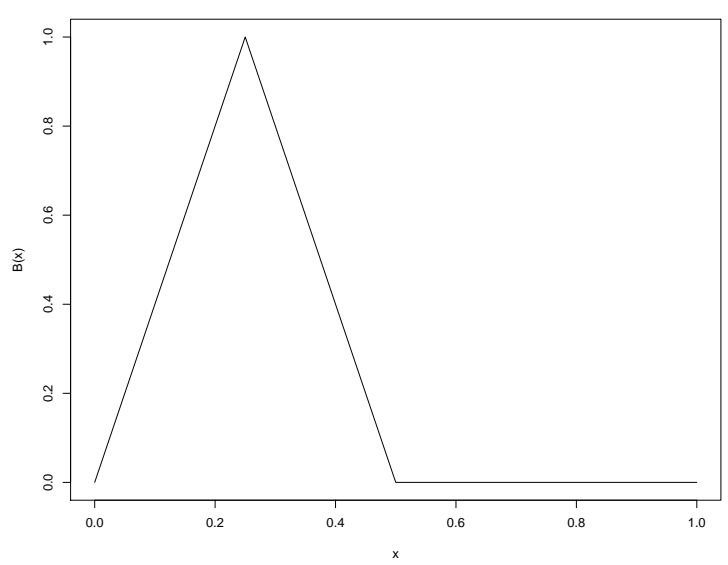

(a)

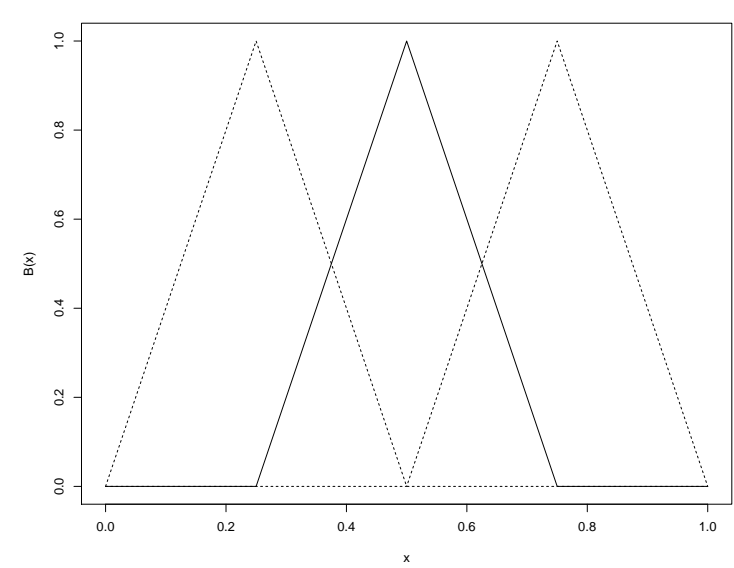

(b)

Figura 2.2: (a) B-spline de grau 1 considerando cinco nós. (b) B-splines de grau 1 considerando os mesmos cinco nós.

Se aumentarmos o grau dos B-splines para 2, obtemos os B-splines quadráticos. Na Figura 2.3 apresentamos alguns exemplos considerando os nós nos pontos $[0 ; 0,2 ; 0,4 ; 0,6 ; 0,8 ; 1,0]$. Nota-se na Figura 2.3a que três pedaços de polinômios de grau 2, unidos por dois nós interiores (no exemplo, 0,2 e 0,4), formam um B-spline quadrático, sendo que à esquerda do primeiro nó e à direita do último nó, o B-spline assume valor 0. Na Figura 2.3b são apresentados os outros B-splines quadráticos possíveis considerando os mesmos seis nós.

Alguns exemplos de B-splines cúbicos considerando nós nos pontos $0,0,1,0,2,0,3,0,4,0,5$, 0,6, 0,7, 0, 8, 0, 9 e 1 são apresentados na Figura 2.4. Um B-spline cúbico é formado por quatro pedaços de polinômios de grau 3 que são unidos em três nós interiores. Na Figura 2.4a, vemos que os polinômios são conectados nos nós $0,1,0,2$ e 0,3 , sendo que à esquerda do primeiro nó (no exemplo, 0) e à direita do último nó (no exemplo, 0,4) o B-spline cúbico assume valor 0. Na Figura $2.4 \mathrm{~b}$ podemos observar mais B-splines cúbicos formados pelos mesmos onze nós. Nota-se que, como estamos calculando B-splines cúbicos utilizando onze nós, temos sete B-splines possíveis.

Os B-splines com nós equidistantes possuem uma desvantagem: a quantidade de suavização só pode ser influenciada pelo número de nós que será utilizado, que geralmente é pequeno. Isso porque se for utilizado um número grande de nós a curva obtida fica superajustada aos dados.

Uma forma de resolver esse problema é utilizar o método P-splines, proposto por Eilers e Marx (1996) e que será utilizado neste trabalho.

Os P-splines, ou splines penalizados, consistem em penalizar as diferenças entre os coeficientes 


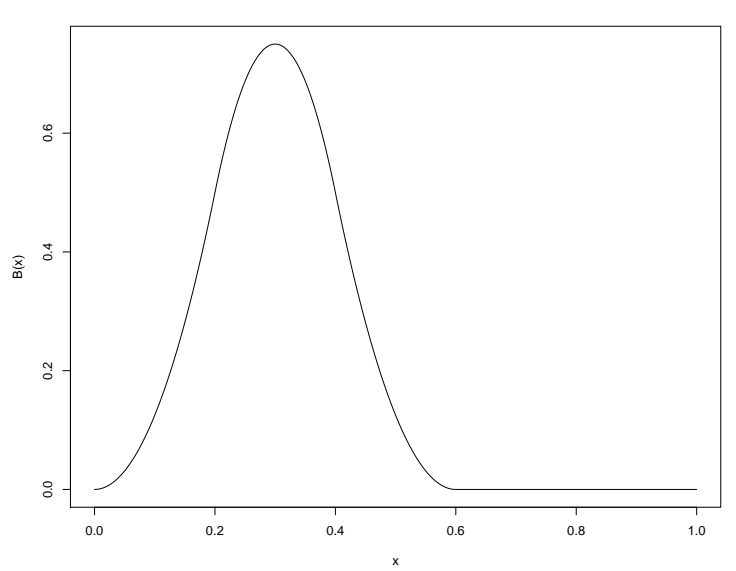

(a)

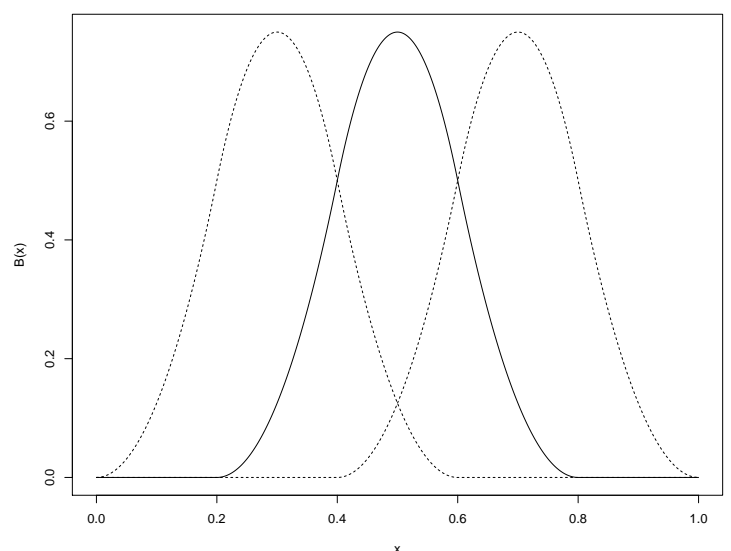

(b)

Figura 2.3: (a) B-spline quadrático considerando seis nós. (b) B-splines quadráticos considerando os mesmos seis nós.



(a)

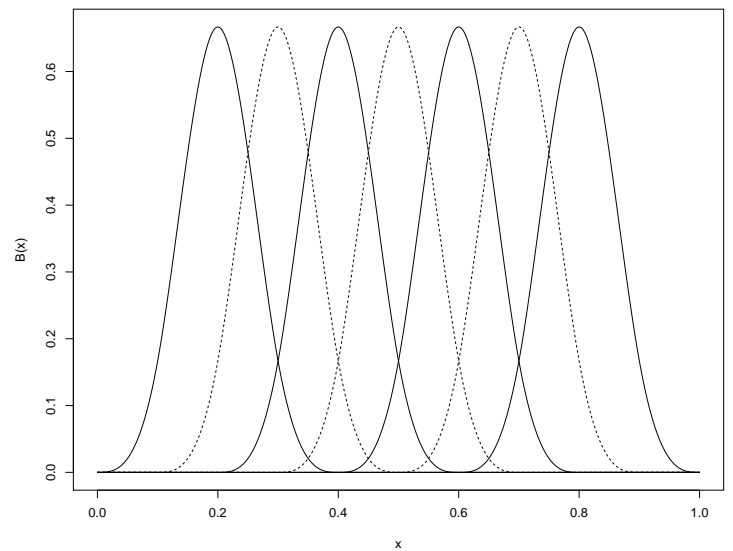

(b)

Figura 2.4: (a) B-spline cúbico considerando onze nós. (b) B-splines cúbicos considerando os mesmos onze nós.

dos B-splines adjacentes, fazendo com que a flexibilidade dos B-splines seja reduzida. Isso permite que seja escolhido um número grande de nós, por exemplo, entre 10 a 20 nós, pois o superajuste causado por essa escolha é tratado por este método.

Desta forma, os P-splines nada mais são do que uma combinação entre os B-splines e uma função de penalização de diferenças de ordem $d, \Delta^{d}$, aplicada aos coeficientes adjacentes estimados $\gamma_{j}$ dos B-splines.

As diferenças $\Delta^{d} \gamma_{j}$ são definidas por

$$
\Delta \gamma_{j}=\gamma_{j}-\gamma_{j-1} ; \Delta^{2} \gamma_{j}=\gamma_{j}-2 \gamma_{j-1}+\gamma_{j-2} ; \Delta^{d} \gamma_{j}=\Delta\left(\Delta^{d-1} \gamma_{j}\right), \text { com } j=1, \ldots, q
$$

em que $d$ é a ordem das diferenças.

Com relação à posição dos nós, Eilers e Marx (1996) sugerem que o primeiro nó seja fixado no 
ponto em que o primeiro B-spline deixa de ser zero. Já o número de nós a ser utilizado pode ser determinado de diversas maneiras. Uma delas é selecionar diferentes números de nós e obter para cada um a validação cruzada generalizada (VCG), de forma que o número de nós escolhido será o que minimizar essa quantidade. Para mais detalhes sobre a escolha do número de nós, consultar Ruppert (2002).

Na Seção 2.4 discutiremos com mais detalhes sobre o critério de penalização utilizado nos Psplines que será aplicado neste trabalho. Além disso, definiremos outro tipo de penalização aplicado a splines cúbicos.

\subsection{Critério de penalização}

Como dito anteriormente, a escolha de um grande número de nós para os B-splines faz com que a curva fique superajustada aos dados, chegando até mesmo a interpolar. Nesse caso, a qualidade do ajuste será ótima, porém a curva obtida será pouco suave e a sua variabilidade será tão alta quanto a variabilidade de $\mathbf{y}$. Em contrapartida, se a curva obtida for muito suave, há perda de informação e o ajuste pode não representar os dados da maneira que esperamos.

Ao acrescentar um critério de penalização no logaritmo da função de verossimilhança, a suavidade da curva é controlada por um coeficiente $\lambda$ chamado de parâmetro de suavização. Dessa forma, resolvemos o problema de superajuste nos dados e obtemos o melhor ajuste possível.

Assim, temos que a função de penalização dos P-splines é dada por

$$
\gamma^{\top} \mathbf{P}_{d} \gamma
$$

em que $\gamma$ são os coeficientes dos B-splines, $\mathbf{P}_{d}=\left(\Delta^{d}\right)^{\top}\left(\Delta^{d}\right)$ e $\Delta^{d}$ é o operador de diferenças de ordem $d$, para $d=1,2, \ldots$

Por exemplo, para $d=1$, temos que a função penalização é definida por

$$
\boldsymbol{\gamma}^{\top} \boldsymbol{\Delta}^{\top} \boldsymbol{\Delta} \boldsymbol{\gamma}=\left(\gamma_{2}-\gamma_{1}\right)^{2}+\left(\gamma_{3}-\gamma_{2}\right)^{2}+\ldots+\left(\gamma_{q}-\gamma_{q-1}\right)^{2},
$$

em que o operador de diferenças de tamanho $q \times q$ fica dado por

$$
\boldsymbol{\Delta}=\left[\begin{array}{ccccc}
-1 & 1 & 0 & 0 & \ldots \\
0 & -1 & 1 & 0 & \ldots \\
0 & 0 & 0 & -1 & \ldots \\
\vdots & \vdots & \vdots & \vdots & \ddots
\end{array}\right]
$$

$\mathrm{e}$

$$
\boldsymbol{\gamma}^{\top} \boldsymbol{\Delta}^{\top} \boldsymbol{\Delta} \boldsymbol{\gamma}=\boldsymbol{\gamma}^{\top}\left[\begin{array}{ccccc}
1 & -1 & 0 & 0 & \ldots \\
-1 & 2 & -1 & 0 & \ldots \\
0 & -1 & 2 & -1 & \ldots \\
\vdots & \vdots & \vdots & \vdots & \ddots
\end{array}\right] \gamma
$$

Adotando a ordem $d=2$ para o operador de diferenças, a penalização fica dada por 


$$
\boldsymbol{\gamma}^{\top}\left(\boldsymbol{\Delta}^{2}\right)^{\top}\left(\boldsymbol{\Delta}^{2}\right) \boldsymbol{\gamma}=\left(\gamma_{1}-2 \gamma_{2}-\gamma_{3}\right)^{2}+\left(\gamma_{2}-2 \gamma_{3}+\gamma_{4}\right)^{2}+\ldots+\left(\gamma_{q-2}-2 \gamma_{q-1}+\gamma_{q}\right)
$$

e o operador de diferenças $\Delta^{2}$ assume a forma

$$
\boldsymbol{\Delta}^{2}=\left[\begin{array}{ccccc}
1 & -2 & 1 & 0 & \ldots \\
0 & 1 & -2 & 1 & \ldots \\
0 & 0 & 1 & -2 & \ldots \\
\vdots & \vdots & \vdots & \vdots & \ddots
\end{array}\right]
$$

Outra forma de penalização é aplicada diretamente na curva $f($.$) . Esse critério avalia a integral$ da derivada de segunda ordem em todo o domínio da variável $t \in[a, b]$ e foi introduzida por Reinsch (1967). No caso dos splines cúbicos, a penalização fica dada por

$$
\int_{a}^{b}\left[f^{\prime \prime}(t)\right]^{2} d t
$$

Green e Silverman (1994) mostram que podemos expressar a penalidade acima da seguinte maneira:

$$
\int_{a}^{b}\left[f^{\prime \prime}(t)\right]^{2} d t=\mathbf{f}^{\top} \mathbf{K} \mathbf{f}
$$

em que $\mathbf{f}=\left(f\left(t_{1}^{0}\right), \ldots, f\left(t_{q}^{0}\right)\right)^{\top}$ são os parâmetros a serem estimados, sendo $t_{j}^{0}$ os valores ordenados e distintos da variável explicativa $t_{i}$ controlada de forma não paramétrica, com $j=1, \ldots, q$ e $\mathbf{K}=\mathbf{Q R}^{-1} \mathbf{Q}^{\top}$.

Sendo $h_{i}=t_{i+1}-t_{i}$, para $i=1, \ldots, n-1$, temos que $\mathbf{Q}$ é uma matriz $n \times(n-2)$ composta pelos elementos $q_{i j}$, para $i=1, \ldots, n$ e $j=2, \ldots, n-1$, dados por

$$
\begin{gathered}
q_{(j-1) j}=h_{j-1}^{-1}, \\
q_{j j}=-h_{j-1}^{-1}-h_{j}^{-1} \mathrm{e} \\
q_{(j+1) j}=h_{j}^{-1}
\end{gathered}
$$

para $j=2, \ldots, n-1$ e $q_{i j}=0$ para $|i-j| \geq 2$.

Já, a matriz $\mathbf{R}$ é simétrica de dimensão $(n-2) \times(n-2)$ com elementos $r_{i j}$ que são definidos da seguinte maneira:

$$
\begin{gathered}
r_{i i}=\frac{1}{3}\left(h_{i-1}+h_{i}\right), \text { para } i=2, \ldots, n-1, \\
r_{i(i+1)}=r_{(i+1) i}=\frac{1}{6} h_{i}, \text { para } i=2, \ldots, n-2 \mathrm{e} \\
r_{i j}=0, \text { para }|i-j| \geq 2 .
\end{gathered}
$$

A vantagem de aplicar uma penalização diretamente nos coeficientes ao invés de aplicá-la na curva, como é feito dos splines cúbicos, é a possibilidade de redução da dimensionalidade do problema. Além disso, a penalização P-splines baseada em diferenças é mais flexível, pois independe 
do grau adotado para os B-splines, de forma que é possível combinar qualquer ordem da penalidade com qualquer ordem das bases de De Boor.

Os P-splines têm propriedades úteis que foram parcialmente herdadas dos B-splines. Primeiramente, os P-splines não mostram efeitos de fronteira, ou seja, a extensão de uma curva ajustada ou de uma densidade fora do domínio físico dos dados geralmente se inclina em direção a zero, como foi mostrado nas Figuras 2.2, 2.3 e 2.4. Além disso, P-splines podem ajustar dados polinomiais de forma exata, isto é, dado $\left(x_{i}, y_{i}\right)$ e sendo $y_{i}$ um polinômio de grau $k$ em $x$, então os P-splines com penalidade de grau $k+1$ ou maior vão ajustar exatamente os dados, independente do valor de $\lambda$. Por fim, uma propriedade que é especialmente útil para suavizar densidades é que os $\mathrm{P}$-splines podem conservar os momentos dos dados, ou seja, a média e a variância da densidade estimada serão iguais às dos dados. Para mais detalhes, consultar Eilers e Marx (1996).

A penalidade usual utilizada nos P-splines é baseada em diferenças. Porém, é possível utilizar outras penalidades discretas para obter ajustes com as propriedades desejadas, sejam elas para garantir a monotonicidade da função, para exigir uma forma convexa ou côncava ou para forçar os sinais das restrições. Por exemplo, para dados periódicos (diários, mensais, lunares, entre outros), há a possibilidade das extremidades da curva não se unirem suavemente quando utilizada uma suavização linear, o que pode ser solucionado utilizando uma penalização circular combinada com bases circulares dos B-splines. Para mais detalhes sobre essas variações, nomeadas de penalidades de delineamento, consultar Eilers e Marx (2010) e Eilers et al. (2015).

\subsubsection{Função de verossimilhança penalizada}

Para os modelos lineares parciais generalizados utilizando P-splines, definido em (2.1), temos que o vetor de parâmetros a ser estimado é $\boldsymbol{\theta}=\left(\boldsymbol{\beta}^{\top}, \boldsymbol{\gamma}^{\top}, \phi\right)^{\top}$.

O logaritmo da função de verossimilhança para os MLPGs é dado por

$$
L(\boldsymbol{\theta})=\sum_{i=1}^{n}\left[\phi\left\{y_{i} \theta_{i}-b\left(\theta_{i}\right)\right\}+c\left(y_{i} ; \phi\right)\right]
$$

em que $\theta_{i}=\theta\left(\mu_{i}\right) \operatorname{com} \mu_{i}=g^{-1}\left(\eta_{i}\right)$ e $\eta_{i}=\mathbf{x}_{i}^{\top} \boldsymbol{\beta}+f\left(t_{i}\right)$.

A maximização direta de (2.3) sem que tenha sido estabelecida qualquer restrição sobre a função não paramétrica $f\left(t_{i}\right)$, pode gerar um superajuste aos dados e fazer com que os parâmetros $\boldsymbol{\beta}$ sejam não identificáveis; vide, por exemplo, Green (1987) e Ibacache-Pulgar (2009). Uma alternativa para enfrentarmos esse problema, como já mencionado na Seção 2.4, é incorporar no logaritmo da função de verossimilhança uma função de penalização, que possibilita maior controle sobre a suavidade do ajuste e evita que ocorra superajuste aos dados. Sendo assim, definimos o logaritmo da função de verossimilhança penalizada, $L_{p}(\boldsymbol{\theta}, \lambda)$, da seguinte forma:

$$
L_{p}(\boldsymbol{\theta}, \lambda)=\sum_{i=1}^{n}\left[\phi\left\{y_{i} \theta_{i}-b\left(\theta_{i}\right)\right\}+c\left(y_{i} ; \phi\right)\right]-\frac{\lambda}{2} \boldsymbol{\gamma}^{\top} \mathbf{P}_{d} \boldsymbol{\gamma},
$$

em que $\lambda>0$ é o parâmetro de suavização, $\mathbf{P}_{d}=\left(\Delta^{d}\right)^{\top}\left(\Delta^{d}\right)$ e $\Delta^{d}$ é o operador de diferença de ordem $d$ conforme definido na Seção 2.4.

Quando $\lambda \rightarrow 0$, temos que a penalização tem pouca influência no modelo, de forma que a prioridade seja o ajuste ótimo da curva. Em contrapartida, quando $\lambda \rightarrow \infty$ a suavidade da curva é priorizada, evitando que ocorra superajuste dos dados. 
Para os splines cúbicos naturais com a penalização baseada na integral da derivada de segunda ordem de $f\left(t_{i}\right)$, temos que o logaritmo da função de verossimilhança penalizada fica dada por

$$
L_{p}(\boldsymbol{\theta}, \lambda)=\sum_{i=1}^{n}\left[\phi\left\{y_{i} \theta_{i}-b\left(\theta_{i}\right)\right\}+c\left(y_{i} ; \phi\right)\right]-\frac{\lambda}{2} \mathbf{f}^{\top} \mathbf{K f} .
$$

A partir da próxima seção, iremos desenvolver os cálculos para os P-splines com a função de penalização definida em (2.2). A extensão dos resultados para o caso de splines cúbicos naturais com a penalização baseada na segunda derivada de $f($.$) não será discutida neste trabalho, mas não$ é difícil de ser obtida (vide, por exemplo, Green e Silverman 1994).

\subsubsection{Função escore e matriz de informação de Fisher penalizadas}

Definido o logaritmo da função de verossimilhança penalizada, $L_{p}(\boldsymbol{\theta}, \lambda)$, podemos obter a função escore e a informação de Fisher penalizadas para os parâmetros $\boldsymbol{\beta}, \boldsymbol{\gamma}$ e $\phi$.

A função escore penalizada de $\boldsymbol{\theta}$ é definida por

$$
\mathbf{U}_{p}^{\boldsymbol{\theta}}(\boldsymbol{\theta})=\left(\begin{array}{c}
\mathbf{U}_{p}^{\boldsymbol{\beta}}(\boldsymbol{\theta}) \\
\mathbf{U}_{p}^{\gamma}(\boldsymbol{\theta}) \\
\mathrm{U}_{p}^{\phi}(\boldsymbol{\theta})
\end{array}\right)
$$

em que $\mathbf{U}_{p}^{\boldsymbol{\beta}}(\boldsymbol{\theta})$ é um vetor de funções escore penalizadas da parte paramétrica do modelo, de dimensão $p \times 1, \mathbf{U}_{p}^{\gamma}(\boldsymbol{\theta})$ é um vetor de funções escore penalizadas referentes ao componente não paramétrico, de dimensão $q \times 1$ e $\mathrm{U}_{p}^{\phi}(\boldsymbol{\theta})$ é a função escore do parâmetro de precisão $\phi$. Essas funções são dadas por

$$
\begin{aligned}
\mathbf{U}_{p}^{\boldsymbol{\beta}}(\boldsymbol{\theta}) & =\frac{\partial L_{p}(\boldsymbol{\theta}, \lambda)}{\partial \boldsymbol{\beta}}, \\
\mathbf{U}_{p}^{\boldsymbol{\gamma}}(\boldsymbol{\theta}) & =\frac{\partial L_{p}(\boldsymbol{\theta}, \lambda)}{\partial \boldsymbol{\gamma}} \mathrm{e} \\
\mathbf{U}_{p}^{\phi}(\boldsymbol{\theta}) & =\frac{\partial L_{p}(\boldsymbol{\theta}, \lambda)}{\partial \phi} .
\end{aligned}
$$

Após algumas manipulações algébricas, apresentadas no Apêndice A, obtemos os seguintes resultados:

$$
\begin{gathered}
\mathbf{U}_{p}^{\boldsymbol{\beta}}(\boldsymbol{\theta})=\phi \mathbf{X}^{\top} \mathbf{W}^{\frac{1}{2}} \mathbf{V}^{-\frac{1}{2}}(\mathbf{y}-\boldsymbol{\mu}), \\
\mathbf{U}_{p}^{\boldsymbol{\gamma}}(\boldsymbol{\theta})=\phi \mathbf{B}^{\top} \mathbf{W}^{\frac{1}{2}} \mathbf{V}^{-\frac{1}{2}}(\mathbf{y}-\boldsymbol{\mu})-\lambda \mathbf{P}_{d} \boldsymbol{\gamma} \mathrm{e} \\
\mathbf{U}_{p}^{\phi}(\boldsymbol{\theta})=\sum_{i=1}^{n}\left\{y_{i} \theta_{i}-b\left(\theta_{i}\right)\right\}+\sum_{i=1}^{n} c^{\prime}\left(y_{i} ; \phi\right),
\end{gathered}
$$

em que $\mathbf{W}=\operatorname{diag}\left\{w_{1}, \ldots, w_{n}\right\}$ é a matriz de pesos $\operatorname{com} w_{i}=\frac{\left(d \mu_{i} / d \eta_{i}\right)^{2}}{V_{i}}, \mathbf{V}=\operatorname{diag}\left\{V_{1}, \ldots, V_{n}\right\} \operatorname{com}$ $V_{i}=\frac{d \mu_{i}}{d \theta_{i}}$ a função de variância, $\mathbf{y}=\left(y_{1}, \ldots, y_{n}\right)^{\top}, \boldsymbol{\mu}=\left(\mu_{1}, \ldots, \mu_{n}\right)^{\top}, \mathbf{X}$ uma matriz de dimensão 
$n \times p$ composta pelos elementos $x_{i j}$, B é uma matriz de dimensão $n \times q$ formada pelos componentes $B_{j}^{(m)}\left(t_{i}\right)$ da base de De Boor, $\lambda>0$ é o parâmetro de suavização, $\mathbf{P}_{d}$ é o operador de diferenças, $\boldsymbol{\gamma}=\left(\gamma_{1}, \ldots, \gamma_{q}\right)^{\top}$ e $c^{\prime}\left(y_{i} ; \phi\right)=\frac{d c\left(y_{i} ; \phi\right)}{d \phi}$, para $i=1, \ldots, n$ e $j=1, \ldots, q$.

A matriz de informação de Fisher penalizada para $\boldsymbol{\theta}$ fica dada por

$$
\mathbf{K}_{p}^{\boldsymbol{\theta} \boldsymbol{\theta}}(\boldsymbol{\theta})=E\left\{-\frac{\partial^{2} L_{p}(\boldsymbol{\theta}, \lambda)}{\partial \boldsymbol{\theta} \partial \boldsymbol{\theta}^{\top}}\right\}
$$

em que

$$
\mathbf{K}_{p}^{\boldsymbol{\theta} \boldsymbol{\theta}}(\boldsymbol{\theta})=\left(\begin{array}{ccc}
\mathbf{K}_{p}^{\boldsymbol{\beta} \boldsymbol{\beta}}(\boldsymbol{\theta}) & \mathbf{K}_{p}^{\boldsymbol{\beta} \gamma}(\boldsymbol{\theta}) & \mathbf{K}_{p}^{\boldsymbol{\beta} \phi}(\boldsymbol{\theta}) \\
\mathbf{K}_{p}^{\gamma \boldsymbol{\beta}}(\boldsymbol{\theta}) & \mathbf{K}_{p}^{\gamma \boldsymbol{\gamma}}(\boldsymbol{\theta}) & \mathbf{K}_{p}^{\gamma \phi}(\boldsymbol{\theta}) \\
\mathbf{K}_{p}^{\phi \boldsymbol{\beta}}(\boldsymbol{\theta}) & \mathbf{K}_{p}^{\phi \boldsymbol{\gamma}}(\boldsymbol{\theta}) & \mathbf{K}_{p}^{\phi \phi}(\boldsymbol{\theta})
\end{array}\right)
$$

$\operatorname{com} \mathbf{K}_{p}^{\boldsymbol{\beta} \gamma}(\boldsymbol{\theta})=\left\{\mathbf{K}_{p}^{\boldsymbol{\gamma} \boldsymbol{\beta}}(\boldsymbol{\theta})\right\}^{\top}, \mathbf{K}_{p}^{\boldsymbol{\beta} \phi}(\boldsymbol{\theta})=\left\{\mathbf{K}_{p}^{\phi \boldsymbol{\beta}}(\boldsymbol{\theta})\right\}^{\top} \mathrm{e} \mathbf{K}_{p}^{\boldsymbol{\gamma}(\boldsymbol{\theta})}=\left\{\mathbf{K}_{p}^{\phi \boldsymbol{\gamma}}(\boldsymbol{\theta})\right\}^{\top}$.

Após algumas manipulações algébricas, apresentadas no Apêndice A, temos os seguintes resultados:

$$
\begin{gathered}
\mathbf{K}_{p}^{\boldsymbol{\beta} \beta}(\boldsymbol{\theta})=E\left\{-\frac{\partial^{2} L_{p}(\boldsymbol{\theta}, \lambda)}{\partial \beta_{j} \partial \beta_{l}}\right\}=\phi \mathbf{X}^{\top} \mathbf{W} \mathbf{X}, \\
\mathbf{K}_{p}^{\gamma \boldsymbol{\gamma}}(\boldsymbol{\theta})=E\left\{-\frac{\partial^{2} L_{p}(\boldsymbol{\theta}, \lambda)}{\partial \gamma_{j} \partial \gamma_{l}}\right\}=\phi \mathbf{B}^{\top} \mathbf{W B}+\lambda \mathbf{P}_{d} \mathrm{e} \\
\mathbf{K}_{p}^{\phi \phi}(\boldsymbol{\theta})=E\left\{-\frac{\partial^{2} L_{p}(\boldsymbol{\theta}, \lambda)}{\partial \phi \partial \phi}\right\}=-\sum_{i=1}^{n} E\left\{c^{\prime \prime}\left(y_{i} ; \phi\right)\right\},
\end{gathered}
$$

em que $c^{\prime \prime}\left(y_{i} ; \phi\right)=\frac{d^{2} c\left(y_{i} ; \phi\right)}{d \phi^{2}}$, para $i=1, \ldots, n$.

Após derivarmos a diagonal da matriz de informação de Fisher penalizada de $\boldsymbol{\theta}$, vamos definir as submatrizes de informação de Fisher com parâmetros cruzados. Sendo assim, temos que

$$
\begin{gathered}
\mathbf{K}_{p}^{\beta_{j} \gamma_{l}}(\boldsymbol{\theta})=E\left\{-\frac{\partial^{2} L_{p}(\boldsymbol{\theta}, \lambda)}{\partial \beta_{j} \partial \gamma_{l}}\right\}=\phi \mathbf{X}^{\top} \mathbf{W B}, \\
\mathrm{K}_{p}^{\beta_{j} \phi}(\boldsymbol{\theta})=E\left\{-\frac{\partial^{2} L_{p}(\boldsymbol{\theta}, \lambda)}{\partial \beta_{j} \partial \phi}\right\}=0 \mathrm{e} \\
\mathrm{K}_{p}^{\gamma_{j} \phi}(\boldsymbol{\theta})=E\left\{-\frac{\partial L_{p}(\boldsymbol{\theta}, \lambda)}{\partial \gamma_{j} \partial \phi}\right\}=0 .
\end{gathered}
$$

Dessa forma, vemos que apenas os parâmetros $\boldsymbol{\beta}$ e $\boldsymbol{\gamma}$ não são ortogonais.

\subsection{Estimação dos parâmetros do modelo}

Para estimarmos os parâmetros do modelo linear parcial generalizado utilizando P-splines é preciso encontrar os valores que maximizam a função de verossimilhança penalizada, ou de forma equivalente, o logaritmo da função de verossimilhança penalizada.

O método que utilizaremos neste trabalho para a estimação de parâmetros é uma combinação entre o processo iterativo de escore de Fisher e o algoritmo backftting (Gauss-Seidel). 
Vamos supor que o logaritmo da função de verossimilhança penalizada definido em (2.4) é uma função côncava e atende a certas condições de regularidade, discutidas para o caso de regressão não paramétrica em $\mathrm{Gu}$ (2013). Os parâmetros $\boldsymbol{\beta}$ e $\boldsymbol{\gamma}$ que maximizam $L_{p}(\boldsymbol{\theta}, \lambda)$ em todo o espaço paramétrico $\boldsymbol{\Theta}$ são chamados de estimadores de máxima verossimilhança penalizada (EMVPs), $\hat{\boldsymbol{\beta}}$ e $\hat{\gamma}$, e satisfazem à seguinte desigualdade:

$$
L_{p}(\hat{\boldsymbol{\theta}}, \lambda) \geq \sup _{\boldsymbol{\theta} \in \Theta} L_{p}(\boldsymbol{\theta}, \lambda) .
$$

Assumindo $\lambda$ e $\phi$ fixos, os EMVPs, $\hat{\boldsymbol{\beta}}$ e $\hat{\boldsymbol{\gamma}}$, são derivados da solução das seguintes equações:

$$
\begin{aligned}
& \mathbf{U}_{p}^{\boldsymbol{\beta}}(\boldsymbol{\theta})=\phi \mathbf{X}^{\top} \mathbf{W}^{\frac{1}{2}} \mathbf{V}^{-\frac{1}{2}}(\mathbf{y}-\boldsymbol{\mu})=0 \quad \mathrm{e} \\
& \mathbf{U}_{p}^{\gamma}(\boldsymbol{\theta})=\phi \mathbf{B}^{\top} \mathbf{W}^{\frac{1}{2}} \mathbf{V}^{-\frac{1}{2}}(\mathbf{y}-\boldsymbol{\mu})-\lambda \mathbf{P}_{d} \boldsymbol{\gamma}=0 .
\end{aligned}
$$

Dadas as expressões acima, podemos utilizar o processo iterativo escore de Fisher que permite resolvê-las. Sendo assim, a $(u+1)$-ésima etapa desse processo iterativo para maximizar a função de verossimilhança penalizada é dada por

$$
\left(\begin{array}{ll}
\mathbf{K}_{p}^{\boldsymbol{\beta} \beta} & \mathbf{K}_{p}^{\boldsymbol{\beta} \gamma} \\
\mathbf{K}_{p}^{\boldsymbol{\gamma} \boldsymbol{\beta}} & \mathbf{K}_{p}^{\boldsymbol{\gamma} \boldsymbol{\gamma}}
\end{array}\right)^{(u)}\left(\begin{array}{c}
\boldsymbol{\beta}^{(u+1)}-\boldsymbol{\beta}^{(u)} \\
\boldsymbol{\gamma}^{(u+1)}-\boldsymbol{\gamma}^{(u)}
\end{array}\right)=\left(\begin{array}{c}
\mathbf{U}_{p}^{\boldsymbol{\beta}} \\
\mathbf{U}_{p}^{\boldsymbol{\gamma}}
\end{array}\right)^{(u)}, \operatorname{para} u=0,1,2, \ldots
$$

Com os resultados obtidos na Seção 2.4 .2 e redefinindo o parâmetro de suavização para $\lambda=$ $\lambda \phi^{-1}$, temos que

$$
\left(\begin{array}{cc}
\mathbf{X}^{\top} \mathbf{W X} & \mathbf{X}^{\top} \mathbf{W B} \\
\mathbf{B}^{\top} \mathbf{W X} & \mathbf{B}^{\top} \mathbf{W B}+\lambda \mathbf{P}_{d}
\end{array}\right)^{(u)}\left(\begin{array}{c}
\boldsymbol{\beta}^{(u+1)}-\boldsymbol{\beta}^{(u)} \\
\boldsymbol{\gamma}^{(u+1)}-\boldsymbol{\gamma}^{(u)}
\end{array}\right)=\left(\begin{array}{c}
\mathbf{X}^{\top} \mathbf{W}^{\frac{1}{2}} \mathbf{V}^{-\frac{1}{2}}(\mathbf{y}-\boldsymbol{\mu}) \\
\mathbf{B}^{\top} \mathbf{W}^{\frac{1}{2}} \mathbf{V}^{-\frac{1}{2}}(\mathbf{y}-\boldsymbol{\mu})-\lambda \mathbf{P}_{d} \boldsymbol{\gamma}
\end{array}\right)^{(u)}
$$

Após algumas manipulações algébricas, podemos reescrever as equações de estimação da seguinte forma:

$$
\begin{gathered}
\boldsymbol{\beta}^{(u+1)}=\left(\mathbf{X}^{\top} \mathbf{W}^{(u)} \mathbf{X}\right)^{-1} \mathbf{X}^{\top} \mathbf{W}^{(u)}\left\{\mathbf{z}^{(u)}-\mathbf{B} \boldsymbol{\gamma}^{(u)}\right\} \mathrm{e} \\
\boldsymbol{\gamma}^{(u+1)}=\left(\mathbf{B}^{\top} \mathbf{W}^{(u)} \mathbf{B}+\lambda \mathbf{P}_{d}\right)^{-1} \mathbf{B}^{\top} \mathbf{W}^{(u)}\left\{\mathbf{z}^{(u)}-\mathbf{X} \boldsymbol{\beta}^{(u)}\right\},
\end{gathered}
$$

em que $\mathbf{z}=\mathbf{W}^{-\frac{1}{2}} \mathbf{V}^{-\frac{1}{2}}(\mathbf{y}-\boldsymbol{\mu})+\mathbf{X} \boldsymbol{\beta}+\mathbf{B} \boldsymbol{\gamma}$, para $u=0,1,2, \ldots$

Podemos interpretar as equações acima de forma intuitiva. Se em (2.15) o valor de $\boldsymbol{\gamma}$ for conhecido e considerado constante, a expressão $\mathbf{B} \gamma=\mathbf{f}(\mathbf{t}), \operatorname{com} \mathbf{f}(\mathbf{t})=\left(f\left(t_{1}\right), \ldots, f\left(t_{n}\right)\right)^{\top}$, é subtraída de $\mathbf{z}$ e podemos estimar $\boldsymbol{\beta}$ pela máxima verossimilhança penalizada considerando essas diferenças. Da mesma maneira, se em (2.16) $\boldsymbol{\beta}$ for conhecido e constante, a parte $\mathbf{X} \boldsymbol{\beta}$ será subtraída de $\mathbf{z}$ e obteremos uma estimativa de máxima verossimilhança penalizada dessas diferenças para $\gamma$.

Podemos estender essa análise de forma que, alternando as equações (2.15) e (2.16), obtemos repetidamente os valores de $\hat{\boldsymbol{\beta}}$ e de $\hat{\boldsymbol{\gamma}}$ até convergir para a estimação de máxima verossimilhança penalizada de cada um desses parâmetros. Esse procedimento é conhecido como algoritmo backfitting e foi introduzido por Breiman e Friedman (1985). Para mais detalhes, também consultar Buja et al. (1989). 
Mostramos na Seção 2.4.2 que os parâmetros $\left(\boldsymbol{\beta}^{\top}, \boldsymbol{\gamma}^{\top}\right)^{\top}$ e $\phi$ são ortogonais. Sendo assim, podemos estimar esse parâmetro a partir de um processo iterativo considerando as estimativas obtidas para $\hat{\boldsymbol{\beta}}$ e $\hat{\boldsymbol{\gamma}}$ através do algoritmo backfitting. O processo iterativo para $\phi$ é dado por

$$
\begin{aligned}
& \phi^{(s+1)}=\phi^{(s)}+\left[\left\{\mathrm{K}_{p}^{\phi \phi}(\boldsymbol{\theta})\right\}^{-1} \mathrm{U}_{p}^{\phi}(\boldsymbol{\theta})\right]^{(s)} \\
& \phi^{(s+1)}=\phi^{(s)}+\left(\frac{\sum_{i=1}^{n}\left\{y_{i} \theta_{i}-b\left(\theta_{i}\right)\right\}+\sum_{i=1}^{n} c^{\prime}\left(y_{i} ; \phi\right)}{\left[-\sum_{i=1}^{n} E\left\{c^{\prime \prime}\left(y_{i} ; \phi\right)\right\}\right]}\right)^{(s)},
\end{aligned}
$$

em que $s=0,1, \ldots$ O processo iterativo (2.15) e (2.16) deve alternar com (2.17) até que a convergência seja alcançada, ou seja, haverá iterações até que as funções individuais não mudem.

A $(u+1)$-ésima etapa do algoritmo backfitting para estimar os parâmetros $\boldsymbol{\beta}, \boldsymbol{\gamma}$ e $\phi$ é descrita a seguir.

(i) Adotar valores iniciais $\boldsymbol{\beta}^{(\mathbf{0})}, \boldsymbol{\gamma}^{(\mathbf{0})}$ e $\phi^{(0)}$ para os parâmetros. Podemos, por exemplo, considerar as estimativas de $\boldsymbol{\beta}^{(\mathbf{0})}$ e $\phi^{(0)}$ do modelo linear generalizado paramétrico e atribuir $\gamma^{(\mathbf{0})}=0$.

(ii) Para $\phi$ e $\lambda$ fixados, executar o processo iterativo descrito em (2.15) e (2.16) até a convergência.

(iii) Obtidas as estimativas para $\boldsymbol{\beta}$ e $\boldsymbol{\gamma}$ no passo anterior, executar o processo iterativo descrito em (2.17) até a convergência.

(iv) Repetir o passo 2 e o passo 3 até obter a convergência global, ou seja, até que a os resultados obtidos do passo $(i v)^{(a)}$ sejam praticamente iguais aos obtidos no passo $(i v)^{(a-1)}$.

Sob certas condições relacionadas às propriedades das matrizes $\mathbf{W}, \mathbf{B}$ e $\mathbf{P}_{d}$, como $\mathbf{W}$ ser simétrica positiva definida, Green e Silverman (1994) mostram que o algoritmo backfitting sempre converge.

\subsubsection{Graus de liberdade efetivos}

Os graus de liberdade efetivos correspondem ao custo total de estimação dos parâmetros dos componentes paramétrico e não paramétrico do modelo. Em princípio, temos $p+q$ parâmetros para serem estimados no preditor linear $\boldsymbol{\eta}=\mathbf{X} \boldsymbol{\beta}+\mathbf{B} \boldsymbol{\gamma}$, sendo que este valor corresponderia ao custo total de graus de liberdade para a estimação desses parâmetros. Contudo, como há uma penalização na função de verossimilhança que implica em uma redução do espaço paramétrico, particularmente do componente não paramétrico, este custo pode ser bem menor. Assim, a determinação dos graus de liberdade efetivos vai depender de uma definição do custo da estimação.

Hastie e Tibshirani 1990 (cap. 6; vide também Green e Silverman 1994; cap. 4) definem os graus de liberdade efetivos com base na solução do preditor linear $\boldsymbol{\eta}$ similarmente ao caso linear, usando projetores lineares ou suavizadores. Assim, para os MLPGs temos que

$$
\hat{\boldsymbol{\eta}}=\mathbf{X} \hat{\boldsymbol{\beta}}+\mathbf{B} \hat{\boldsymbol{\gamma}}=\hat{\mathbf{H}}(\lambda) \hat{\mathbf{z}}
$$

em que $\mathbf{H}(\lambda)$ é um suavizador definido por (vide demonstração no Apêndice C)

$$
\mathbf{H}(\lambda)=\left[\begin{array}{ll}
\mathbf{X} & \mathbf{B}
\end{array}\right]\left[\begin{array}{cc}
\mathbf{X}^{\top} \mathbf{W} \mathbf{X} & \mathbf{X}^{\top} \mathbf{W B} \\
\mathbf{B}^{\top} \mathbf{W} \mathbf{X} & \mathbf{B}^{\top} \mathbf{W} \mathbf{B}+\lambda \mathbf{P}_{d}
\end{array}\right]^{-1}\left[\begin{array}{c}
\mathbf{X}^{\top} \\
\mathbf{B}^{\top}
\end{array}\right] \mathbf{W} .
$$


Seguindo Hastie e Tibshirani 1990 (cap.6) os graus de liberdade efetivos nos MLPGs são definidos por

$$
d f(\lambda)=\operatorname{tr}\{\widehat{\mathbf{H}}(\lambda)\}
$$

ou seja, a soma dos autovalores do suavizador $\widehat{\mathbf{H}}(\lambda)$. Similarmente a Green e Silverman 1994 (p. 90) podemos decompor o suavizador $\mathbf{H}(\lambda)$ da seguinte forma:

$$
\mathbf{H}(\lambda)=\mathbf{H}_{\beta}(\lambda)+\mathbf{H}_{\gamma}(\lambda)
$$

em que

$$
\mathbf{H}_{\beta}(\lambda)=\left\{\mathbf{I}_{n}-\mathbf{H}_{\gamma}(\lambda)\right\} \mathbf{X}\left[\mathbf{X}^{\top} \mathbf{W}\left\{\mathbf{I}_{n}-\mathbf{H}_{\gamma}(\lambda)\right\} \mathbf{X}\right]^{-1} \mathbf{X}^{\top} \mathbf{W}\left\{\mathbf{I}_{n}-\mathbf{H}_{\gamma}(\lambda)\right\}
$$

e

$$
\mathbf{H}_{\gamma}(\lambda)=\mathbf{B}\left(\mathbf{B}^{\top} \mathbf{W B}+\lambda \mathbf{P}_{d}\right)^{-1} \mathbf{B}^{\top} \mathbf{W}
$$

Logo, teremos

$$
d f(\lambda)=\operatorname{tr}\left\{\widehat{\mathbf{H}}_{\beta}(\lambda)\right\}+\operatorname{tr}\left\{\widehat{\mathbf{H}}_{\gamma}(\lambda)\right\} .
$$

Como $\mathbf{H}_{\beta}(\lambda)$ não é uma matriz idempotente, $\operatorname{tr}\left\{\widehat{\mathbf{H}}_{\beta}(\lambda)\right\} \neq p$. Porém, há estudos em Eubank (1999) (vide também Noda, 2013) em que $\mathbf{H}_{\beta}(\lambda)$ se aproxima de uma matriz idempotente. Nesses $\operatorname{casos} \operatorname{tr}\left\{\mathbf{H}_{\beta}(\lambda)\right\} \cong p \mathrm{e}$

$$
d f(\lambda) \cong p+d f_{\gamma}(\lambda)
$$

em que $d f_{\gamma}(\lambda)=\operatorname{tr}\left\{\widehat{\mathbf{H}}_{\gamma}(\lambda)\right\}$. Usaremos essa definição para $d f(\lambda)$ neste trabalho.

Portanto, para o caso dos modelos lineares parciais generalizados, temos que a quantidade de parâmetros a serem estimados será aproximadamente dada por $1+p+d f_{\gamma}(\lambda)$, em que 1 se refere ao parâmetro $\phi, p$ aos parâmetros $\boldsymbol{\beta}$ e $d f_{\gamma}(\lambda)$ aos parâmetros da função não paramétrica.

Temos que

$$
\begin{aligned}
d f_{\gamma}(\lambda) & =\operatorname{tr}\left\{\widehat{\mathbf{H}}_{\gamma}(\lambda)\right\} \\
& =\operatorname{tr}\left\{\mathbf{B}\left(\mathbf{B}^{\top} \widehat{\mathbf{W}} \mathbf{B}+\lambda \mathbf{P}_{d}\right)^{-1} \mathbf{B}^{\top} \widehat{\mathbf{W}}\right\} .
\end{aligned}
$$

Note que quando $\lambda=0$, temos que $\operatorname{tr}\left\{\widehat{\mathbf{H}}_{\gamma}(\lambda)\right\}=q$. Assim, é mais vantajoso computacionalmente utilizar a seguinte equação:

$$
d f_{\gamma}(\lambda)=\operatorname{tr}\left\{\left(\mathbf{B}^{\top} \widehat{\mathbf{W}} \mathbf{B}+\lambda \mathbf{P}_{d}\right)^{-1} \mathbf{B}^{\top} \widehat{\mathbf{W}} \mathbf{B}\right\}
$$

Eilers e Marx (1996) mostram que o $\operatorname{tr}\left\{\widehat{\mathbf{H}}_{\gamma}(\lambda)\right\}$ pode ser reescrito como 


$$
\begin{aligned}
d f_{\gamma}(\lambda) & =\operatorname{tr}\left\{\widehat{\mathbf{H}}_{\gamma}(\lambda)\right\} \\
& =\operatorname{tr}\left\{\mathbf{B}^{\top} \widehat{\mathbf{W}} \mathbf{B}\left(\mathbf{B}^{\top} \widehat{\mathbf{W}} \mathbf{B}+\lambda \mathbf{P}_{d}\right)^{-1}\right\} \\
& =\operatorname{tr}\left\{\left(\mathbf{I}+\lambda \mathbf{Q}^{-\frac{1}{2}} \mathbf{P}_{d} \mathbf{Q}^{-\frac{1}{2}}\right)^{-1}\right\} \\
& =\sum_{j=1}^{q} \frac{1}{1+\lambda \alpha_{j}},
\end{aligned}
$$

em que $\alpha_{j}$ são os autovalores da matriz não negativa definida $\mathbf{Q}^{-\frac{1}{2}} \mathbf{P}_{d} \mathbf{Q}^{-\frac{1}{2}}$ e $\mathbf{Q}^{\frac{1}{2}}$ é uma matriz positiva definida, de forma que $\mathbf{B}^{\top} \widehat{\mathbf{W}} \mathbf{B}=\mathbf{Q}^{\frac{1}{2}} \mathbf{Q}^{\frac{1}{2}}$. Portanto, temos uma relação inversa entre os graus de liberdade efetivos e o parâmetro de suavização. Logo, sabendo o valor de um desses parâmetros podemos obter o outro. Na prática é menos complicado estabelecer uma faixa de valores para os graus de liberdade efetivos do que para o parâmetro de suavização. Assim, se conseguirmos expressar $\lambda$ em função de $d f(\lambda)$ poderemos aplicar com mais eficiência os métodos descritos a seguir para estimar o parâmetro de suavização.

Wood 2017 (seção 5.4.2) faz uma interpretação intuitiva dos graus de liberdade efetivos atribuídos a $\hat{\gamma}$ em uma comparação com o estimador irrestrito (sem penalização), que denotaremos por $\tilde{\gamma}$.

É possível mostrar, após algumas decomposições matriciais, que

$$
\hat{\gamma}_{j}=\left(1+\lambda \alpha_{j}\right)^{-1} \tilde{\gamma}_{j}
$$

para $j=1, \ldots, q$. Ou seja, há um encolhimento ("shrinkage") do estimador $\hat{\gamma}_{j}$, uma vez que o fator $\left(1+\lambda \alpha_{j}\right)^{-1}$ está no intervalo $(0,1]$. Como é atribuído 1 grau de liberdade para $\tilde{\gamma}_{j}$ é razoável pensar que $\left(1+\lambda \alpha_{j}\right)^{-1}$ sejam os graus de liberdade efetivos para $\hat{\gamma}_{j}$. Logo, os graus de liberdade efetivos para $\hat{\gamma}=\left(\hat{\gamma}_{1}, \ldots, \hat{\gamma}_{q}\right)^{\top}$ seriam dados pela soma $\sum_{j=1}^{q}\left(1+\lambda \alpha_{j}\right)^{-1}$.

\subsubsection{Estimação do parâmetro de suavização}

Como já dito anteriormente, o parâmetro de suavização $\lambda$ tem papel fundamental para a obtenção do melhor ajuste. Isso porque esse parâmetro pondera a quantidade de suavização com o melhor ajuste possível para os dados, fazendo com que o modelo não interpole, mas também não fique suave a ponto de não representar os dados. Por conta disso é necessário métodos para encontrar o valor ótimo desse parâmetro.

Vamos apresentar duas formas de estimar $\lambda$, o método de validação cruzada (VC) apresentado por Wahba e Wold (1975) e o método de validação cruzada generalizada (VCG), descrito por Craven e Wahba (1979).

O método de validação cruzada utiliza parte dos dados para o ajuste do modelo e o restante para avaliar a adequabilidade do ajuste. Green e Silverman (1994) e Wood (2017) definem esse método por

$$
\mathrm{VC}(\lambda)=\frac{1}{n} \sum_{i=1}^{n} \frac{\hat{w}_{i}\left(\hat{z}_{i}-x_{i}^{\top} \hat{\beta}\right)^{2}}{\left\{1-\hat{h}_{i i}(\lambda)\right\}^{2}},
$$

em que as estimativas são obtidas por meio do processo iterativo dado um valor fixo de $\lambda$ e $\hat{h}_{i i}$ são os elementos da matriz $\hat{\mathbf{H}}(\lambda)$. Assim, o $\lambda$ ótimo escolhido será aquele que minimize $\operatorname{VC}(\lambda)$. 
O método de validação cruzada generalizada é uma versão modificada da validação cruzada apresentada anteriormente. A modificação é no termo $\hat{h}_{i i}(\lambda)$, que é substituído pela média do traço da estimativa do suavizador $\mathbf{H}(\lambda)$, de forma que a definição da VCG fica dada por

$$
\operatorname{VCG}(\lambda)=\sum_{i=1}^{n} \frac{\hat{w}_{i}\left(\hat{z}_{i}-x_{i}^{\top} \hat{\beta}\right)^{2}}{\left[1-n^{-1} \operatorname{tr}\{\hat{\mathbf{H}}(\lambda)\}\right]^{2}} .
$$

Assim como na validação cruzada, o valor ótimo de $\lambda$ será o que minimizar VCG $(\lambda)$. Para mais detalhes sobre a validação cruzada generalizada, ver também Wahba (1985).

Além desses métodos, pode-se estimar $\lambda$ aplicando medidas de informação, como o Critério de Informação de Akaike (AIC), ver Akaike (1974), e o Critério de Informação Bayesiano (BIC), ver Schwarz (1978), para os modelos parcialmente lineares.

O método AIC corrige o logaritmo da função de verossimilhança do modelo ajustado de forma que seja considerado apenas o número efetivo de parâmetros, ou seja, o método busca o melhor ajuste com o menor número de parâmetros possíveis. Isso é feito penalizando o logaritmo da função de verossimilhança com duas vezes o número de parâmetros do modelo. Sendo assim, o critério de informação de Akaike é definido da seguinte forma:

$$
\operatorname{AIC}(\lambda)=-2 L_{p}(\hat{\boldsymbol{\theta}}, \lambda)+2\left\{p+1+d f_{\gamma}(\lambda)\right\} .
$$

Similarmente, o método BIC é dado por

$$
\operatorname{BIC}(\lambda)=-2 L_{p}(\hat{\boldsymbol{\theta}}, \lambda)+\log (n)\left\{p+1+d f_{\gamma}(\lambda)\right\},
$$

em que $L_{p}(\hat{\boldsymbol{\theta}}, \lambda)$ é o logaritmo da função de verossimilhança penalizada avaliada em $\hat{\boldsymbol{\theta}}$.

O valor ótimo de $\lambda$ nesses casos será o valor que minimiza as expressões (2.20) ou (2.21).

O traço é uma boa ferramenta para comparar diferentes ajustes, pois permite avaliar o quanto o ajuste foi suavizado utilizando diferentes números de nós, graus dos B-splines e ordens das penalidades de diferenças.

Não havendo um ajuste adequado com $\operatorname{AIC}(\lambda)$ e $\operatorname{BIC}(\lambda)$ segundo, por exemplo, uma análise de resíduos, pode-se aplicar o critério $\operatorname{GAIC}(\lambda)=-2 L_{p}(\hat{\boldsymbol{\theta}}, \lambda)+k\left\{p+1+d f_{\gamma}(\lambda)\right\}$, para $k=2, \ldots, \log (n)$, até obter um ajuste adequado e, consequentemente, o valor de $\lambda$. Esse critério está disponível na biblioteca gamlss.

\subsection{Resultados Assintóticos}

Conforme obtido na Seção 2.4.2, temos que a matriz de informação de Fisher penalizada é dada por

$$
\mathbf{K}_{p}^{\boldsymbol{\theta} \boldsymbol{\theta}}(\boldsymbol{\theta})=\left(\begin{array}{ccc}
\mathbf{K}_{p}^{\boldsymbol{\beta} \boldsymbol{\beta}}(\boldsymbol{\theta}) & \mathbf{K}_{p}^{\boldsymbol{\beta} \gamma}(\boldsymbol{\theta}) & 0 \\
\mathbf{K}_{p}^{\boldsymbol{\gamma} \boldsymbol{\beta}}(\boldsymbol{\theta}) & \mathbf{K}_{p}^{\gamma \boldsymbol{\gamma}}(\boldsymbol{\theta}) & 0 \\
0 & 0 & \mathrm{~K}_{p}^{\phi \phi}(\boldsymbol{\theta})
\end{array}\right)
$$

Sendo assim, a partir de propriedades da inversa de matrizes em blocos, $\left[\mathbf{K}_{p}^{\boldsymbol{\theta} \theta}(\boldsymbol{\theta})\right]^{-1}$ é definida por 


$$
\left[\mathbf{K}_{p}^{\boldsymbol{\theta} \boldsymbol{\theta}}(\boldsymbol{\theta})\right]^{-1}=\left(\begin{array}{ccc}
\mathbf{I} & \mathbf{J} & 0 \\
\mathbf{L} & \mathbf{M} & 0 \\
0 & 0 & {\left[\mathrm{~K}_{p}^{\phi \phi}(\boldsymbol{\theta})\right]^{-1}}
\end{array}\right)
$$

em que

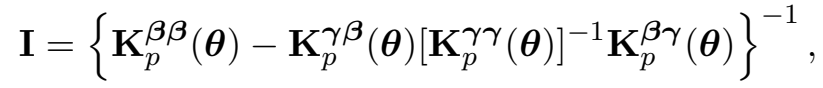

$$
\begin{aligned}
& \mathbf{J}=-\left[\mathbf{K}_{p}^{\boldsymbol{\beta} \boldsymbol{\beta}}(\boldsymbol{\theta})\right]^{-1} \mathbf{K}_{p}^{\boldsymbol{\gamma} \boldsymbol{\beta}}(\boldsymbol{\theta})\left\{\mathbf{K}_{p}^{\boldsymbol{\gamma} \boldsymbol{\gamma}}(\boldsymbol{\theta})-\mathbf{K}_{p}^{\boldsymbol{\beta} \boldsymbol{\gamma}}(\boldsymbol{\theta})\left[\mathbf{K}_{p}^{\boldsymbol{\beta} \boldsymbol{\beta}}(\boldsymbol{\theta})\right]^{-1} \mathbf{K}_{p}^{\boldsymbol{\gamma} \boldsymbol{\beta}}(\boldsymbol{\theta})\right\}^{-1}, \\
& \mathbf{L}=-\left[\mathbf{K}_{p}^{\boldsymbol{\gamma} \boldsymbol{\gamma}}(\boldsymbol{\theta})\right]^{-1} \mathbf{K}_{p}^{\boldsymbol{\beta} \boldsymbol{\gamma}}(\boldsymbol{\theta})\left\{\mathbf{K}_{p}^{\boldsymbol{\beta} \boldsymbol{\beta}}(\boldsymbol{\theta})-\mathbf{K}_{p}^{\boldsymbol{\gamma} \boldsymbol{\beta}}(\boldsymbol{\theta})\left[\mathbf{K}_{p}^{\boldsymbol{\gamma} \boldsymbol{\gamma}}(\boldsymbol{\theta})\right]^{-1} \mathbf{K}_{p}^{\boldsymbol{\beta} \boldsymbol{\gamma}}(\boldsymbol{\theta})\right\}^{-1} \mathrm{e} \\
& \mathbf{M}=\left\{\mathbf{K}_{p}^{\gamma \boldsymbol{\gamma}}(\boldsymbol{\theta})-\mathbf{K}_{p}^{\boldsymbol{\beta} \gamma}(\boldsymbol{\theta})\left[\mathbf{K}_{p}^{\boldsymbol{\beta} \boldsymbol{\beta}}(\boldsymbol{\theta})\right]^{-1} \mathbf{K}_{p}^{\boldsymbol{\gamma} \boldsymbol{\beta}}(\boldsymbol{\theta})\right\}^{-1} .
\end{aligned}
$$

Os parâmetros $\left(\boldsymbol{\beta}^{\top}, \boldsymbol{\gamma}^{\top}\right)^{\top}$ são assintoticamente ortogonais a $\phi$, porém os parâmetros $\boldsymbol{\beta}$ e $\boldsymbol{\gamma}$ não são ortogonais assintoticamente.

A teoria assintótica em modelos semiparamétricos depende da fixação do número de nós. Quando o número de nós cresce com o tamanho da amostra $n$ as condições de regularidade da teoria assintótica paramétrica não podem mais serem aplicadas. Contudo, quando o número de nós é fixado como é o caso dos P-splines, podemos adaptar a teoria assintótica do caso paramétrico para o caso semiparamétrico. Conforme observado por Yu e Ruppert (2002) é bastante razoável supor que $\lambda \rightarrow 0$ à medida que $n$ cresce, porém a utilização da matriz de Fisher não penalizada $\mathbf{K}_{\boldsymbol{\theta} \boldsymbol{\theta}}(\boldsymbol{\theta})$ para extrair as variâncias e covariâncias dos estimadores $\left(\hat{\boldsymbol{\beta}}^{\top}, \hat{\boldsymbol{\gamma}}^{\top}, \hat{\phi}\right)^{\top}$ pode levar a valores superestimados. Assim, uma proposta (vide Vanegas e Paula, 2016) é a utilização de um estimador sandwich para a matriz de variância-covariância assintótica de $\hat{\boldsymbol{\theta}}$, definido por

$$
\mathbf{V}_{\boldsymbol{\theta} \boldsymbol{\theta}}=\left[\mathbf{K}_{p}^{\boldsymbol{\theta} \boldsymbol{\theta}}(\boldsymbol{\theta})\right]^{-1} \mathbf{K}_{\boldsymbol{\theta} \boldsymbol{\theta}}(\boldsymbol{\theta})\left[\mathbf{K}_{p}^{\boldsymbol{\theta} \boldsymbol{\theta}}(\boldsymbol{\theta})\right]^{-1}
$$

em que

$$
\mathbf{K}_{\boldsymbol{\theta} \boldsymbol{\theta}}(\boldsymbol{\theta})=\left(\begin{array}{ccc}
\mathbf{K}_{\boldsymbol{\beta} \boldsymbol{\beta}}(\boldsymbol{\theta}) & \mathbf{K}_{\boldsymbol{\beta} \boldsymbol{\gamma}}(\boldsymbol{\theta}) & 0 \\
\mathbf{K}_{\boldsymbol{\gamma} \boldsymbol{\beta}}(\boldsymbol{\theta}) & \mathbf{K}_{\boldsymbol{\gamma} \boldsymbol{\gamma}}(\boldsymbol{\theta}) & 0 \\
0 & 0 & \mathrm{~K}_{\phi \phi}(\boldsymbol{\theta})
\end{array}\right)
$$

$\operatorname{com} \mathbf{K}_{\boldsymbol{\beta} \boldsymbol{\beta}}(\boldsymbol{\theta})=\mathbf{K}_{p}^{\boldsymbol{\beta} \boldsymbol{\beta}}(\boldsymbol{\theta}), \mathbf{K}_{\boldsymbol{\beta} \boldsymbol{\gamma}}(\boldsymbol{\theta})=\mathbf{K}_{p}^{\boldsymbol{\beta} \boldsymbol{\gamma}}(\boldsymbol{\theta}), \mathbf{K}_{\boldsymbol{\gamma} \boldsymbol{\beta}}(\boldsymbol{\theta})=\mathbf{K}_{p}^{\boldsymbol{\gamma} \boldsymbol{\beta}}(\boldsymbol{\theta}), \mathrm{K}_{\phi \phi}(\boldsymbol{\theta})=\mathrm{K}_{p}^{\phi \phi}(\boldsymbol{\theta})$ e $\mathbf{K}_{\boldsymbol{\gamma} \boldsymbol{\gamma}}(\boldsymbol{\theta})=$ $\phi \mathbf{B}^{\top} \mathbf{W B}$.

Para $n$ grande, pode-se mostrar que $\hat{\boldsymbol{\theta}}$ se aproxima de uma normal $p^{*}$-variada de vetor de médias $\boldsymbol{\theta}$ e matriz de variância-covariância $\mathbf{V}_{\boldsymbol{\theta} \boldsymbol{\theta}}$, em que $p^{*}=p+q+1$. Contudo, a recomendação é utilizar essa matriz apenas para extrair os erros padrão assintóticos. Na aplicação de testes de hipóteses para os componentes não paramétricos deve-se levar em conta os graus de liberdade efetivos $d f(\lambda)$. Wood (2017) usa argumentos de Inferência Bayesiana para recomendar a extração dos erros padrão assintóticos, bem como covariâncias assintóticas de $\hat{\boldsymbol{\theta}}$, da matriz de variância-covariância penalizada assintótica $\mathbf{K}_{p}^{\boldsymbol{\theta} \theta}(\boldsymbol{\theta})$. 


\subsubsection{Teste de hipóteses}

Supor que temos interesse em testar uma hipótese na forma $H_{0}: \mathbf{C} \boldsymbol{\beta}=\mathbf{0}$ contra $H_{1}: \mathbf{C} \boldsymbol{\beta} \neq \mathbf{0}$ em que $\mathbf{C}$ é uma matriz $k \times p \operatorname{com} k \leq p$. Sejam $\hat{\boldsymbol{\theta}}^{0}$ e $\hat{\boldsymbol{\theta}}$ as estimativas de máxima verossimilhança penalizadas sob $H_{0}$ e $H_{1}$, respectivamente. Assim, segundo Wood 2017 (p. 313), a estatística da razão de verossimilhanças

$$
R V=2\left\{L_{p}\left(\hat{\boldsymbol{\theta}}, \lambda_{1}\right)-L_{p}\left(\hat{\boldsymbol{\theta}}^{0}, \lambda_{0}\right)\right\}
$$

segue, sob $H_{0}$, distribuição qui-quadrado com graus de liberdade dados por $d f\left(\lambda_{1}\right)-d f\left(\lambda_{0}\right)$.

Conforme definido na Seção 2.2, sabemos que a função não paramétrica utilizada neste trabalho é dada pelos P-splines de forma que $f\left(t_{i}\right)=\sum_{j=1}^{q} B_{j}^{(m)}\left(t_{i}\right) \gamma_{j}=\mathbf{B} \boldsymbol{\gamma}$. Para avaliar se a variável explicativa modelada de forma não paramétrica é significativa é necessário realizar teste de hipótese.

Sendo assim, vamos considerar as hipóteses $H_{0}: \gamma=0$ contra $H_{1}: \gamma \neq 0$. Considerando a matriz de variância-covariância derivada na seção anterior, segundo Vanegas e Paula (2016), o efeito da função não paramétrica pode ser considerado como insignificante, quando a estatística apresentada a seguir assume valores pequenos

$$
W_{\gamma}=\hat{\gamma}^{\top} \widehat{\operatorname{Var}}^{-1}[\hat{\gamma}] \hat{\gamma}
$$

que, sob a hipótese nula, segue assintoticamente uma distribuição $\chi_{q}^{2}$. Como a penalização leva a uma redução do espaço paramétrico de $\gamma$, os graus de liberdade referentes a essa estatística tipo Wald devem ser na prática muito menores do que $q$. Wood 2017 (cap. 6) observa que usar $q$ pode levar a uma perda de poder e sugere, entre outras propostas, utilizar $d f_{\gamma}(\lambda)$ no lugar de $q$.

\subsection{Análise de resíduos}

Para verificar a adequabilidade de um modelo estatístico são realizadas análises de diagnóstico. Uma das técnicas mais utilizadas é baseada em resíduos.

Os resíduos ordinários $\epsilon_{i}$ são definidos pela diferença entre o valor observado da variável resposta e o valor estimado, $r_{i}=y_{i}-\hat{y}_{i}$, para $i=1, \ldots, n$. Na regressão normal linear, os resíduos seguem uma distribuição normal com média zero, podendo ser padronizados para que tenham variâncias iguais. No caso dos MLGs, por exemplo, um dos resíduos mais utilizados é o resíduo de Pearson, definido como

$$
r_{p, i}=\frac{y_{i}-\hat{\mu}_{i}}{\sqrt{V(\hat{\mu})}}
$$

em que $\hat{\mu}_{i}$ é o valor ajustado para $\mu_{i}$. Porém, para o caso em que a regressão é não normal, como no GAMLSS que possui uma variedade de distribuições a serem utilizadas, essa definição usual de resíduos pode não se adequada, dado que a suposição de normalidade pode ser rejeitada. Sendo assim, neste trabalho, iremos utilizar como alternativa para essa classe de modelos as análises de diagnóstico baseadas nos resíduos quantílicos aleatorizados, propostos por Dunn e Smyth (1996).

Os resíduos quantílicos são obtidos a partir da inversão da função de distribuição ajustada em cada valor da variável resposta de forma a encontrar o quantil da normal padrão equivalente. Sendo assim, para o caso em que a distribuição assumida no modelo é contínua, temos que esses resíduos 
são dados por

$$
r_{q, i}=\Phi^{-1}\left\{F\left(y_{i} ; \hat{\mu}_{i}\right)\right\},
$$

em que $\Phi$ é a função de distribuição acumulada da normal padrão e $F\left(y_{i} ; \mu_{i}\right)$ é a função de distribuição acumulada da distribuição da variável resposta independente assumida para $y_{i}$, para $i=1, \ldots, n$.

Para o caso em que a distribuição do modelo é discreta, os resíduos quantílicos aleatorizados são definidos da seguinte maneira:

$$
r_{q, i}=\Phi^{-1}\left\{u_{i}\right\},
$$

em que $u_{i}$ é uma variável aleatória uniforme no intervalo $\left(a_{i}, b_{i}\right]$, com $a_{i}=F\left(y_{i}-1 ; \hat{\mu}_{i}\right)$ e $b_{i}=$ $F\left(y_{i} ; \hat{\mu}_{i}\right)$, para $i=1, \ldots, n$.

Considerando que o modelo ajustado está especificado corretamente, podemos afirmar que os resíduos quantílicos seguem uma distribuição aproximadamente normal à medida que $n$ cresce.

\subsection{Considerações}

Este capítulo teve como principal objetivo estudar a teoria dos modelos lineares parciais generalizados. Sendo assim, apresentamos a definição do modelo, discutimos sobre o método de suavização utilizado para a função não paramétrica, derivamos o logaritmo da função de verossimilhança penalizada, as funções escore penalizadas e as submatrizes da matriz de informação de Fisher penalizada. Além disso, apresentamos a estimação dos componentes paramétrico e não paramétrico pela combinação do processo iterativo de Fisher e o algoritmo backfitting e definimos métodos para obter a estimação do parâmetro de suavização, seja por validação cruzada ou medidas de informação. Por fim, discutimos um pouco a teoria assintótica, teste de hipóteses e análise de resíduos nesta classe de modelos. 


\section{Capítulo 3}

\section{Modelos lineares parciais aditivos generalizados}

No capítulo anterior discutimos a teoria dos MLPGs, em que consideramos para o ajuste da parte não paramétrica do modelo o método de suavização por meio de P-splines. Nesses modelos consideramos apenas uma função não paramétrica para ajustar uma variável explicativa contínua. Porém, podemos estender essa definição para o caso em que mais de uma variável explicativa contínua é ajustada de forma não paramétrica.

A ideia surgiu de uma generalização dos MLGs, em que Hastie e Tibshirani (1990) propuseram a utilização da soma de funções desconhecidas ao invés das funções lineares dos preditores, como é feito nos MLGs. Essa nova classe foi denominada de modelos aditivos generalizados (MAGs).

Os modelos lineares parciais aditivos generalizados (MLPAGs) são uma extensão tanto do modelo apresentado no Capítulo 2, quanto dos modelos aditivos generalizados. Isso porque os MLPAGs são utilizados quando a relação da média da variável resposta com algumas variáveis explicativas tem forma paramétrica, ao mesmo tempo em que a relação entre a média da variável resposta e o restante das covariáveis pode ser não linear, combinando os componentes paramétricos e não paramétricos em um mesmo modelo.

Dentre as principais referências para esses modelos estão Hastie e Tibshirani (1990), que apresentam um estudo extenso sobre os modelos aditivos generalizados, métodos de suavização e modelos semiparamétricos. Wood (2017) também desenvolve a teoria de modelos lineares generalizados, modelos aditivos generalizados, métodos de suavização, porém com enfoque na aplicação desses modelos no software R por meio do pacote $m g c v$, desenvolvido pelo mesmo autor. Já Ribgy e Stasinopoulos (2005) desenvolveram o GAMLSS (Generalized Additive Models for Location, Scale and Shape), que relaxam algumas suposições dos MAGs e dos MLGs, além de permitir a modelagem de assimetria e de curtose. Além disso, os autores desenvolveram o pacote gamlss no software R que será utilizado nas aplicações deste trabalho, ver também Stasinopoulos e Ribgy (2007).

Dessa forma, neste capítulo vamos apresentar a teoria dos modelos lineares parciais aditivos generalizados utilizando o método de suavização P-splines. Para isso, apresentamos na Seção 3.1 a especifição desse modelo e na Seção 3.2 definimos o critério de penalização a ser utilizado, obtemos o logaritmo da função de verossimilhança penalizada e derivamos as funções escore e informação de Fisher penalizadas. Na Seção 3.3 apresentamos o método para obter as estimativas dos parâmetros do modelo, além de definir os graus de liberdade efetivos do ajuste e os métodos para estimar o 
parâmetro de suavização. Por fim, na Seção 3.4 são apresentados testes de hipóteses.

\subsection{Especificação do modelo}

Os modelos lineares parciais aditivos generalizados são definidos por

$$
\begin{gathered}
y_{i} \mid\left(\mathbf{x}_{i}, \mathbf{t}_{i}\right) \stackrel{i n d}{\sim} \mathrm{FE}\left(\mu_{i}, \phi\right) \mathrm{com} \\
g\left(\mu_{i}\right)=\eta_{i}=\mathbf{x}_{i}^{\top} \boldsymbol{\beta}+f_{1}\left(t_{i 1}\right)+\ldots+f_{r}\left(t_{i r}\right),
\end{gathered}
$$

em que $\eta_{i}$ é o preditor linear, $g($.$) é a função de ligação, \boldsymbol{\beta}=\left(\beta_{1}, \ldots, \beta_{p}\right)^{\top}$ é o vetor de coeficientes a serem estimados, $\phi$ denota o parâmetro de precisão, $\mathbf{x}_{i}=\left(x_{i 1}, \ldots, x_{i p}\right)^{\top}$ contém valores das variáveis explicativas tratadas de forma paramétrica, $t_{i 1}, \ldots, t_{i r}$ são os valores das covariáveis contínuas que contribuem de forma não paramétrica, $f_{1}(),. \ldots, f_{r}($.$) são funções contínuas e diferenciáveis, para$ $i=1, \ldots, n$, e FE denota a família exponencial.

Como dito anteriormente, as funções não paramétricas podem ser ajustadas de diversas formas e um dos métodos mais utilizados é através de splines. Para os MLPAGs continuaremos adotando o método de suavização por P-splines.

Sendo assim, as funções não paramétricas serão denotadas por

$$
f_{l}\left(t_{i l}\right)=\sum_{j=1}^{q_{l}} B_{j l}^{\left(m_{l}\right)}\left(t_{i l}\right) \gamma_{j l},
$$

em que $q_{l}$ é a dimensão da base do $l$-ésimo P-spline, $k_{l}=q_{l}+m_{l}+1$ é o número de nós, $B_{j l}^{\left(m_{l}\right)}\left(t_{i l}\right)$ denota o $j$-ésimo componente da base do $l$-ésimo spline que é um B-spline de ordem $m_{l}+1$ no ponto $t_{i l}$ e $\gamma_{j l}$ são os coeficientes para serem estimados, para $i=1, \ldots, n, l=1, \ldots, r$ e $j=1, \ldots, q_{l}$. Uma aplicação desse método em modelos aditivos generalizados pode ser encontrado, por exemplo, em Marx e Eilers (1998).

Podemos reescrever a parte sistemática (3.1) da seguinte maneira:

$$
g\left(\mu_{i}\right)=\eta_{i}=\mathbf{x}_{i}^{\top} \boldsymbol{\beta}+\mathbf{b}_{i 1}^{\top} \boldsymbol{\gamma}_{1}+\ldots+\mathbf{b}_{i r}^{\top} \boldsymbol{\gamma}_{r},
$$

em que $\mathbf{b}_{i l}=\left(B_{1 l}^{\left(m_{l}\right)}\left(t_{i l}\right), \ldots, B_{q_{l} l}^{\left(m_{l}\right)}\left(t_{i l}\right)\right)^{\top}$ e $\gamma_{l}=\left(\gamma_{1 l}, \ldots, \gamma_{q_{l} l}\right)^{\top}$.

Em forma matricial obtemos

$$
\mathbf{g}(\boldsymbol{\mu})=\boldsymbol{\eta}=\mathbf{X} \boldsymbol{\beta}+\sum_{l=1}^{r} \mathbf{B}_{l} \gamma_{l},
$$

em que $\mathbf{X}$ é uma matriz de dimensão $n \times p$ formada pelos valores $x_{i j}$ e $\mathbf{B}_{l}$ é uma matriz de dimensão $n \times q_{l}$ formada pelos valores $B_{j l}^{\left(m_{l}\right)}\left(t_{i l}\right)$, para $i=1, \ldots, n, l=1, \ldots, r$ e $j=1, \ldots, q_{l}$.

\subsection{Critério de penalização}

Do mesmo modo que nos MLPGs, o acréscimo de uma função de penalização no logaritmo da função de verossimilhança permite que a suavidade da curva seja controlada por meio do parâmetro 
de suavização, evitando que o modelo fique superajustado aos dados. Sendo assim, vamos considerar o método de suavização por meio de P-splines.

A função de penalização utilizada nos P-splines é baseada nas diferenças dos coeficientes adjacentes dos B-splines e dada por

$$
\gamma_{l}^{\top} \mathbf{P}_{l}^{d} \gamma_{l}
$$

em que $\gamma_{l}$ são os coeficientes dos B-splines da l-ésima função não paramétrica, $\mathbf{P}_{l}^{d}=\left(\Delta_{l}^{d}\right)^{\top}\left(\Delta_{l}^{d}\right)$ e $\Delta_{l}^{d}$ é o operador de diferenças de ordem $d, l=1, \ldots, r$.

No logaritmo da função de verossimilhança penalizada, as funções de penalização serão controladas pelos parâmetros de suavização $\lambda_{1}, \ldots, \lambda_{r}$.

\subsubsection{Função de verossimilhança penalizada}

Seja $\boldsymbol{\theta}=\left(\boldsymbol{\beta}^{\top}, \boldsymbol{\gamma}_{1}^{\top}, \ldots, \boldsymbol{\gamma}_{r}^{\top}, \phi\right)^{\top}$ o vetor de parâmetros a serem estimados no modelo linear parcial aditivo generalizado, definido em (3.1). Temos que o logaritmo da função de verossimilhança não penalizada dos MLPAGs fica dado por

$$
L(\boldsymbol{\theta})=\sum_{i=1}^{n}\left[\phi\left\{y_{i} \theta_{i}-b\left(\theta_{i}\right)\right\}+c\left(y_{i} ; \phi\right)\right],
$$

em que $g\left(\mu_{i}\right)=\mathbf{x}_{i}^{\top} \boldsymbol{\beta}+\sum_{l=1}^{r} f_{l}\left(t_{i l}\right)$.

Assim como ocorre no MLPGs, maximizar (3.2) sem impor qualquer restrição sobre as funções $f_{l}\left(t_{i l}\right)$, para $l=1, \ldots, r$, pode gerar um superajuste aos dados e causar problemas de identificabilidade dos parâmetros (vide Wood, 2017 e Vanegas e Paula, 2016). Uma maneira de contornar esse problema, assim como já mencionado na Seção 3.2, é acrescentar uma função de penalização ao logaritmo da função de verossimilhança, evitando que o modelo interpole os dados. Assim, definimos o logaritmo da função de verossimilhança penalizada, $L_{p}\left(\boldsymbol{\theta}, \lambda_{1}, \ldots, \lambda_{r}\right)$ da seguinte forma:

$$
L_{p}(\boldsymbol{\theta}, \boldsymbol{\lambda})=\sum_{i=1}^{n}\left[\phi\left\{y_{i} \theta_{i}-b\left(\theta_{i}\right)\right\}+c\left(y_{i} ; \phi\right)\right]-\sum_{l=1}^{r} \frac{\lambda_{l}}{2} \boldsymbol{\gamma}_{l}^{\top} \mathbf{P}_{l}^{d} \boldsymbol{\gamma}_{l},
$$

em que $\boldsymbol{\lambda}=\left(\lambda_{1}, \ldots, \lambda_{r}\right)^{\top}$ é o vetor de parâmetros de suavização, de forma que $\lambda_{l}>0$, com $l=1, \ldots, r$.

Da mesma maneira que nos MLPGs, quando $\lambda_{l} \rightarrow 0$ temos que a penalização tem pouca influência no modelo, priorizando a medida de qualidade do ajuste. Em contrapartida, quando $\lambda_{l} \rightarrow \infty$ a suavização da curva é priorizada, evitando que ocorra superajuste dos dados.

\subsubsection{Função escore e matriz de informação de Fisher penalizada}

Uma vez definido o logaritmo da função de verossimilhança penalizada $L_{p}(\boldsymbol{\theta}, \boldsymbol{\lambda})$, podemos obter a função escore e a matriz de informação de Fisher penalizadas para os parâmetros $\boldsymbol{\beta}, \boldsymbol{\gamma}_{1}, \ldots, \boldsymbol{\gamma}_{r}$ e $\phi$.

O vetor de funções escore penalizadas, $\mathbf{U}_{p}^{\boldsymbol{\theta}}(\boldsymbol{\theta})$, é dado por 


$$
\mathbf{U}_{p}^{\boldsymbol{\theta}}(\boldsymbol{\theta})=\left(\begin{array}{c}
\mathbf{U}_{p}^{\boldsymbol{\beta}}(\boldsymbol{\theta}) \\
\mathbf{U}_{p}^{\gamma_{1}}(\boldsymbol{\theta}) \\
\vdots \\
\mathbf{U}_{p}^{\gamma_{r}}(\boldsymbol{\theta}) \\
\mathbf{U}_{p}^{\phi}(\boldsymbol{\theta})
\end{array}\right)
$$

em que $\mathbf{U}_{p}^{\boldsymbol{\beta}}(\boldsymbol{\theta})$ é um vetor de funções escore penalizadas da parte paramétrica do modelo, de dimensão $p \times 1, \mathbf{U}_{p}^{\gamma_{1}}(\boldsymbol{\theta}), \ldots, \mathbf{U}_{p}^{\gamma_{r}}(\boldsymbol{\theta})$ são as funções escore penalizadas referentes aos componentes não paramétricos, cada uma de dimensão $q_{l} \times 1$ e $\mathrm{U}_{p}^{\phi}(\boldsymbol{\theta})$ é a função escore penalizada do parâmetro de precisão. Essas funções são definidas como

$$
\begin{gathered}
\mathbf{U}_{p}^{\boldsymbol{\beta}}(\boldsymbol{\theta})=\frac{\partial L_{p}(\boldsymbol{\theta}, \boldsymbol{\lambda})}{\partial \boldsymbol{\beta}}, \\
\mathbf{U}_{p}^{\gamma_{l}}(\boldsymbol{\theta})=\frac{\partial L_{p}(\boldsymbol{\theta}, \boldsymbol{\lambda})}{\partial \boldsymbol{\gamma}_{l}} \mathrm{e} \\
\mathrm{U}_{p}^{\phi}(\boldsymbol{\theta})=\frac{\partial L_{p}(\boldsymbol{\theta}, \boldsymbol{\lambda})}{\partial \phi} .
\end{gathered}
$$

Após algumas manipulações algébricas, apresentadas no Apêndice B, obtemos os seguintes resultados:

$$
\begin{gathered}
\mathbf{U}_{p}^{\boldsymbol{\beta}}(\boldsymbol{\theta})=\phi \mathbf{X}^{\top} \mathbf{W}^{\frac{1}{2}} \mathbf{V}^{-\frac{1}{2}}(\mathbf{y}-\boldsymbol{\mu}), \\
\mathbf{U}_{p}^{\gamma_{l}}(\boldsymbol{\theta})=\phi \mathbf{B}_{l}^{\top} \mathbf{W}^{\frac{1}{2}} \mathbf{V}^{-\frac{1}{2}}(\mathbf{y}-\boldsymbol{\mu})-\lambda_{l} \mathbf{P}_{l}^{d} \boldsymbol{\gamma}_{l} \mathrm{e} \\
\mathbf{U}_{p}^{\phi}(\boldsymbol{\theta})=\sum_{i=1}^{n}\left\{y_{i} \theta_{i}-b\left(\theta_{i}\right)\right\}+\sum_{i=1}^{n} c^{\prime}\left(y_{i} ; \phi\right),
\end{gathered}
$$

$\operatorname{com} l=1, \ldots, r$ e $c^{\prime}\left(y_{i} ; \phi\right)=\frac{d c\left(y_{i} ; \phi\right)}{d \phi}$.

Após a derivação das funções escore, vamos obter a matriz de informação de Fisher para $\boldsymbol{\theta}$ que é definida por

$$
\mathbf{K}_{p}^{\boldsymbol{\theta} \boldsymbol{\theta}}(\boldsymbol{\theta})=E\left\{-\frac{\partial^{2} L_{p}(\boldsymbol{\theta}, \boldsymbol{\lambda})}{\partial \boldsymbol{\theta} \partial \boldsymbol{\theta}^{\top}}\right\}
$$

em que

$$
\mathbf{K}_{p}^{\boldsymbol{\theta} \boldsymbol{\theta}}(\boldsymbol{\theta})=\left(\begin{array}{ccccc}
\mathbf{K}_{p}^{\boldsymbol{\beta} \boldsymbol{\beta}}(\boldsymbol{\theta}) & \mathbf{K}_{p}^{\boldsymbol{\beta} \boldsymbol{\gamma}_{1}}(\boldsymbol{\theta}) & \ldots & \mathbf{K}_{p}^{\boldsymbol{\beta} \boldsymbol{\gamma}_{r}}(\boldsymbol{\theta}) & \mathbf{K}_{p}^{\boldsymbol{\beta} \phi}(\boldsymbol{\theta}) \\
\mathbf{K}_{p}^{\boldsymbol{\gamma}_{1} \boldsymbol{\beta}}(\boldsymbol{\theta}) & \mathbf{K}_{p}^{\gamma_{1} \boldsymbol{\gamma}_{1}}(\boldsymbol{\theta}) & \ldots & \mathbf{K}_{p}^{\boldsymbol{\gamma}_{1} \boldsymbol{\gamma}_{\boldsymbol{r}}}(\boldsymbol{\theta}) & \mathbf{K}_{p}^{\boldsymbol{\gamma}_{1} \phi}(\boldsymbol{\theta}) \\
\vdots & \vdots & \ddots & \vdots & \vdots \\
\mathbf{K}_{p}^{\boldsymbol{\gamma}_{r} \boldsymbol{\beta}}(\boldsymbol{\theta}) & \mathbf{K}_{p}^{\gamma_{r} \boldsymbol{\gamma}_{1}}(\boldsymbol{\theta}) & \ldots & \mathbf{K}_{p}^{\boldsymbol{\gamma}_{r} \boldsymbol{\gamma}_{r}}(\boldsymbol{\theta}) & \mathbf{K}_{p}^{\boldsymbol{\gamma}_{r} \phi}(\boldsymbol{\theta}) \\
\mathbf{K}_{p}^{\phi \boldsymbol{\beta}}(\boldsymbol{\theta}) & \mathbf{K}_{p}^{\phi \boldsymbol{\gamma}_{1}}(\boldsymbol{\theta}) & \ldots & \mathbf{K}_{p}^{\phi \boldsymbol{\gamma}_{r}}(\boldsymbol{\theta}) & \mathrm{K}_{p}^{\phi \phi}(\boldsymbol{\theta})
\end{array}\right)
$$

sendo que $\mathbf{K}_{p}^{\boldsymbol{\beta} \gamma_{l}}(\boldsymbol{\theta})=\left\{\mathbf{K}_{p}^{\boldsymbol{\gamma}_{l} \boldsymbol{\beta}}(\boldsymbol{\theta})\right\}^{\top}, \mathbf{K}_{p}^{\boldsymbol{\beta} \phi}(\boldsymbol{\theta})=\left\{\mathbf{K}_{p}^{\phi \boldsymbol{\beta}}(\boldsymbol{\theta})\right\}^{\top}, \mathbf{K}_{p}^{\boldsymbol{\gamma}_{l} \boldsymbol{\gamma}_{l^{\prime}}}(\boldsymbol{\theta})=\left\{\mathbf{K}_{p}^{\boldsymbol{\gamma}_{l^{\prime}} \gamma_{l}}(\boldsymbol{\theta})\right\}^{\top}$ e $\mathbf{K}_{p}^{\boldsymbol{\gamma}_{l} \phi}(\boldsymbol{\theta})=$ $\left\{\mathbf{K}_{p}^{\phi \gamma_{l}}(\boldsymbol{\theta})\right\}^{\top}$, para $l=1, \ldots, r$. 
Os resultados obtidos após algumas manipulações algébricas, também presentes no Apêndice B, são dados por

$$
\begin{gathered}
\mathbf{K}_{p}^{\boldsymbol{\beta} \beta}(\boldsymbol{\theta})=E\left\{-\frac{\partial^{2} L(\boldsymbol{\theta}, \boldsymbol{\lambda})}{\partial \beta_{j} \partial \beta_{l}}\right\}=\phi \mathbf{X}^{\top} \mathbf{W} \mathbf{X} \\
\mathbf{K}_{p}^{\gamma_{l} \gamma_{l}}(\boldsymbol{\theta})=E\left\{-\frac{\partial^{2} L(\boldsymbol{\theta}, \boldsymbol{\lambda})}{\partial \gamma_{j l} \partial \gamma_{k l}}\right\}=\phi \mathbf{B}_{l}^{\top} \mathbf{W} \mathbf{B}_{l}+\lambda_{l} \mathbf{P}_{l}^{d} \mathrm{e} \\
\mathbf{K}_{p}^{\phi \phi}(\boldsymbol{\theta})=E\left\{-\frac{\partial^{2} L(\boldsymbol{\theta}, \boldsymbol{\lambda})}{\partial \phi \partial \phi}\right\}=-\sum_{i=1}^{n} E\left\{c^{\prime \prime}\left(y_{i} ; \phi\right)\right\}
\end{gathered}
$$

para $l=1, \ldots, r$ e $c^{\prime \prime}\left(y_{i} ; \phi\right)=\frac{d^{2} c\left(y_{i} ; \phi\right)}{d \phi^{2}}$.

Vamos agora derivar as matrizes de informação de Fisher penalizadas dos parâmetros cruzados. Dessa forma, obtemos

$$
\begin{gathered}
\mathbf{K}_{p}^{\boldsymbol{\beta} \gamma_{l}}=E\left\{-\frac{\partial^{2} L_{p}(\boldsymbol{\theta}, \boldsymbol{\lambda})}{\partial \beta_{j} \partial \gamma_{k l}}\right\}=\phi \mathbf{X}^{\top} \mathbf{W B}_{l}, \\
\mathrm{~K}_{p}^{\beta_{j} \phi}=E\left\{-\frac{\partial^{2} L_{p}(\boldsymbol{\theta}, \boldsymbol{\lambda})}{\partial \beta_{j} \partial \phi}\right\}=0, \\
\mathbf{K}_{p}^{\gamma_{l} \gamma_{l^{\prime}}}=E\left\{-\frac{\partial^{2} L(\boldsymbol{\theta}, \boldsymbol{\lambda})}{\partial \gamma_{j l} \partial \gamma_{k l^{\prime}}}\right\}=\phi \mathbf{B}_{l}^{\top} \mathbf{W B}_{l^{\prime}} \mathrm{e} \\
\mathbf{K}_{p}^{\gamma_{j l} \phi}=E\left\{-\frac{\partial L(\boldsymbol{\theta}, \lambda)}{\partial \gamma_{j l} \partial \phi}\right\}=E\left\{-\sum_{i=1}^{n} \sqrt{\frac{w_{i}}{V_{i}}}\left(y_{i}-\mu_{i}\right) b_{i j l}\right\}=0,
\end{gathered}
$$

para $l=1, \ldots, r$.

Portanto, temos que apenas os parâmetros $\boldsymbol{\beta}$ e $\gamma_{l}$ e os parâmetros $\gamma_{l}$ e $\gamma_{l^{\prime}}$ não são ortogonais.

Podemos reescrever (3.8) e (3.12) da seguinte maneira:

$$
\mathbf{K}_{p}^{\gamma_{l} \boldsymbol{\gamma}_{l^{\prime}}}= \begin{cases}\phi \mathbf{B}_{l}^{\top} \mathbf{W} \mathbf{B}_{l}+\lambda_{l} \mathbf{P}_{l}^{d}, & \text { se } l=l^{\prime} \\ \phi \mathbf{B}_{l}^{\top} \mathbf{W B}_{l^{\prime}}, & \text { se } l \neq l^{\prime},\end{cases}
$$

$\operatorname{com} l, l^{\prime}=1, \ldots, r$.

\subsection{Estimação dos parâmetros}

Para estimarmos os parâmetros $\boldsymbol{\beta}$ e $\gamma_{l}, l=1, \ldots, r$, vamos utilizar o processo iterativo escore de Fisher e o algoritmo backfitting. Supondo que o logaritmo da função de verossimilhança penalizada $L_{p}(\boldsymbol{\theta}, \boldsymbol{\lambda})$, definido em (3.3), satisfaz certas condições de regularidade, temos que o estimador de máxima verossimilhança penalizada $\hat{\boldsymbol{\theta}}$, satisfaz à seguinte desigualdade:

$$
L_{p}(\hat{\boldsymbol{\theta}}, \boldsymbol{\lambda}) \geq \sup _{\boldsymbol{\theta} \in \Theta} L_{p}(\boldsymbol{\theta}, \boldsymbol{\lambda}) .
$$

Considerando $\boldsymbol{\lambda}$ e $\phi$ fixos, os EMVPs $\hat{\boldsymbol{\beta}}$ e $\hat{\boldsymbol{\gamma}}_{l}$, são derivados da solução das equações abaixo 


$$
\begin{gathered}
\mathbf{U}_{p}^{\boldsymbol{\beta}}(\boldsymbol{\theta})=\phi \mathbf{X}^{\top} \mathbf{W}^{\frac{1}{2}} \mathbf{V}^{-\frac{1}{2}}(\mathbf{y}-\boldsymbol{\mu})=0, \\
\mathbf{U}_{p}^{\boldsymbol{\gamma}_{1}}(\boldsymbol{\theta})=\phi \mathbf{B}_{1}^{\top} \mathbf{W}^{\frac{1}{2}} \mathbf{V}^{-\frac{1}{2}}(\mathbf{y}-\boldsymbol{\mu})-\lambda_{1} \mathbf{P}_{1}^{d} \gamma_{1}=0, \\
\vdots \\
\mathbf{U}_{p}^{\boldsymbol{\gamma}_{r}}(\boldsymbol{\theta})=\phi \mathbf{B}_{r}^{\top} \mathbf{W}^{\frac{1}{2}} \mathbf{V}^{-\frac{1}{2}}(\mathbf{y}-\boldsymbol{\mu})-\lambda_{r} \mathbf{P}_{r}^{d} \boldsymbol{\gamma}_{r}=0 .
\end{gathered}
$$

Green e Silverman (1994) mostram que sob certas condições de regularidade, os estimadores de máxima verossimilhança penalizada existem e são únicos.

Dadas essas expressões, pode-se utilizar o processo iterativo escore de Fisher para resolvê-las. Sendo assim, a $(u+1)$-ésima etapa do processo iterativo para maximixar a função de verossimilhança penalizada é definida por

$$
\left(\begin{array}{cccc}
\mathbf{K}_{p}^{\boldsymbol{\beta} \beta} & \mathbf{K}_{p}^{\boldsymbol{\beta} \boldsymbol{\gamma}_{1}} & \ldots & \mathbf{K}_{p}^{\boldsymbol{\beta} \boldsymbol{\gamma}_{r}} \\
\mathbf{K}_{p}^{\boldsymbol{\gamma}_{1} \boldsymbol{\beta}} & \mathbf{K}_{p}^{\boldsymbol{\gamma}_{1} \boldsymbol{\gamma}_{1}} & \ldots & \mathbf{K}_{p}^{\boldsymbol{\gamma}_{1} \boldsymbol{\gamma}_{r}} \\
\vdots & \vdots & \ddots & \vdots \\
\mathbf{K}_{p}^{\boldsymbol{\gamma}_{r} \boldsymbol{\beta}} & \mathbf{K}_{p}^{\boldsymbol{\gamma}_{r} \boldsymbol{\gamma}_{1}} & \ldots & \mathbf{K}_{p}^{\boldsymbol{\gamma}_{r} \boldsymbol{\gamma}_{r}}
\end{array}\right)^{(u)}\left(\begin{array}{c}
\boldsymbol{\beta}^{(u+1)}-\boldsymbol{\beta}^{(u)} \\
\boldsymbol{\gamma}_{1}^{(u+1)}-\boldsymbol{\gamma}_{1}^{(u)} \\
\vdots \\
\boldsymbol{\gamma}_{r}^{(u+1)}-\boldsymbol{\gamma}_{r}^{(u)}
\end{array}\right)=\left(\begin{array}{c}
\mathbf{U}_{p}^{\boldsymbol{\beta}} \\
\mathbf{U}_{p}^{\boldsymbol{\gamma}_{1}} \\
\vdots \\
\mathbf{U}_{p}^{\boldsymbol{\gamma}_{r}}
\end{array}\right)^{(u)}
$$

Substituindo os valores obtidos na Seção 3.2.2 na equação acima e redefinindo o parâmetro de suavização para $\lambda_{l}=\lambda_{l} \phi^{-1}, l=1, \ldots, r$, obtemos

$$
\begin{gathered}
\left(\begin{array}{cccc}
\mathbf{X}^{\top} \mathbf{W X} & \mathbf{X}^{\top} \mathbf{W} \mathbf{B}_{1} & \ldots & \mathbf{X}^{\top} \mathbf{W} \mathbf{B}_{r} \\
\mathbf{B}_{1}^{\top} \mathbf{W X} & \mathbf{B}_{1}^{\top} \mathbf{W B}_{1}+\lambda_{1} \mathbf{P}_{1}^{d} & \ldots & \mathbf{B}_{1}^{\top} \mathbf{W B}_{r} \\
\vdots & \vdots & \ddots & \vdots \\
\mathbf{B}_{r}^{\top} \mathbf{W X} & \mathbf{B}_{r}^{\top} \mathbf{W B}_{1} & \ldots & \mathbf{B}_{r}^{\top} \mathbf{W} \mathbf{B}_{r}+\lambda_{r} \mathbf{P}_{r}^{d}
\end{array}\right)\left(\begin{array}{c}
\boldsymbol{\beta}^{(u+1)}-\boldsymbol{\beta}^{(u)} \\
\gamma_{1}^{(u+1)}-\gamma_{1}^{(u)} \\
\vdots \\
\boldsymbol{\gamma}_{r}^{(u+1)}-\gamma_{r}^{(u)}
\end{array}\right)= \\
=\left(\begin{array}{c}
\mathbf{X}^{\top} \mathbf{W}^{\frac{1}{2}} \mathbf{V}^{-\frac{1}{2}}(\mathbf{y}-\boldsymbol{\mu}) \\
\mathbf{B}_{1}^{\top} \mathbf{W}^{\frac{1}{2}} \mathbf{V}^{-\frac{1}{2}}(\mathbf{y}-\boldsymbol{\mu})-\lambda_{1} \mathbf{P}_{1}^{d} \boldsymbol{\gamma}_{1} \\
\vdots \\
\mathbf{B}_{r}^{\top} \mathbf{W}^{\frac{1}{2}} \mathbf{V}^{-\frac{1}{2}}(\mathbf{y}-\boldsymbol{\mu})-\lambda_{r} \mathbf{P}_{r}^{d} \boldsymbol{\gamma}_{r}
\end{array}\right)
\end{gathered}
$$

para $u=0,1,2, \ldots$

Após algumas manipulações algébricas, obtemos

$$
\begin{gathered}
\boldsymbol{\beta}^{(u+1)}=\left(\mathbf{X}^{\top} \mathbf{W}^{(u)} \mathbf{X}\right)^{-1} \mathbf{X}^{\top} \mathbf{W}^{(u)}\left\{\mathbf{z}^{(u)}-\sum_{l=1}^{r} \mathbf{B}_{l} \boldsymbol{\gamma}_{l}^{(u)}\right\}, \\
\gamma_{1}^{(u+1)}=\left(\mathbf{B}_{1}^{\top} \mathbf{W}^{(u)} \mathbf{B}_{1}+\lambda_{1} \mathbf{P}_{1}^{d}\right)^{-1} \mathbf{B}_{1}^{\top} \mathbf{W}^{(u)}\left\{\mathbf{z}^{(u)}-\mathbf{X} \boldsymbol{\beta}^{(u)}-\sum_{l=1, l \neq 1}^{r} \mathbf{B}_{l} \gamma_{l}^{(u)}\right\}, \\
\vdots \\
\gamma_{r}^{(u+1)}=\left(\mathbf{B}_{r}^{\top} \mathbf{W}^{(u)} \mathbf{B}_{r}+\lambda_{r} \mathbf{P}_{r}^{d}\right)^{-1} \mathbf{B}_{r}^{\top} \mathbf{W}^{(u)}\left\{\mathbf{z}^{(u)}-\mathbf{X} \boldsymbol{\beta}^{(u)}-\sum_{l=1, l \neq r}^{r} \mathbf{B}_{l} \boldsymbol{\gamma}_{l}^{(u)}\right\},
\end{gathered}
$$

em que $\mathbf{z}=\mathbf{W}^{-\frac{1}{2}} \mathbf{V}^{-\frac{1}{2}}(\mathbf{y}-\boldsymbol{\mu})+\mathbf{X} \boldsymbol{\beta}+\sum_{l=1}^{r} \mathbf{B}_{l} \gamma_{l}$ e $u=0,1,2, \ldots$

Assim como nos MLPGs, resolveremos as equações (3.15), (3.16), ..., (3.17) por meio do algo- 
ritmo backfitting, de forma que, alternando essas equações, os valores para $\hat{\boldsymbol{\beta}}, \hat{\boldsymbol{\gamma}}_{1}, \ldots, \hat{\boldsymbol{\gamma}}_{r}$ são obtidos repetidamente até que se obtenha a convergência.

Além disso, podemos criar um processo iterativo para estimar $\phi$. Como $\left(\boldsymbol{\beta}^{\top}, \boldsymbol{\gamma}_{1}^{\top}, \ldots, \boldsymbol{\gamma}_{r}^{\top}\right)^{\top}$ e $\phi$ são ortogonais (ver Seção 3.2.2), podemos utilizar as estimativas obtidas para $\hat{\boldsymbol{\beta}}, \hat{\boldsymbol{\gamma}_{\mathbf{1}}}, \ldots, \hat{\boldsymbol{\gamma}_{\boldsymbol{r}}}$ por meio do algoritmo backfitting no processo iterativo para estimar $\hat{\phi}$, de forma que o mesmo fica dado por

$$
\begin{aligned}
& \phi^{(s+1)}=\phi^{(s)}+\left\{\left[\mathrm{K}_{p}^{\phi \phi}(\boldsymbol{\theta})\right]^{-1} U_{p}^{\phi}(\boldsymbol{\theta})\right\}^{(s)} \\
& \phi^{(s+1)}=\phi^{(s)}+\left\{\frac{\sum_{i=1}^{n}\left\{y_{i} \theta_{i}-b\left(\theta_{i}\right)\right\}+\sum_{i=1}^{n} c^{\prime}\left(y_{i} ; \phi\right)}{\left(-\sum_{i=1}^{n} E\left\{c^{\prime \prime}\left(y_{i} ; \phi\right)\right\}\right)}\right\}^{(s)},
\end{aligned}
$$

em que $s=0,1,2, \ldots$. Sendo assim, o processo iterativo (3.18) deve alternar com o descrito em (3.15),(3.16), .., (3.17) até que a convergência seja atingida.

A $(u+1)$-ésima etapa do algoritmo backfitting para estimar $\boldsymbol{\theta}$ é definida por

(i) Adotar valores iniciais para os parâmetros, $\boldsymbol{\beta}^{(0)}, \gamma_{1}^{(0)}, \gamma_{2}^{(0)}, \ldots, \gamma_{r}^{(0)}$ e $\phi^{(0)}$. Assim como nos MLPGs, podemos considerar as estimativas de $\boldsymbol{\beta}^{(\mathbf{0})}$ e $\phi^{(0)}$ obtidas no modelo linear generalizado paramétrico e atribuir $\gamma_{l}^{(0)}=0$.

(ii) Para $\phi$ e $\boldsymbol{\lambda}$ fixados, executar o processo iterativo descrito em $(3.15),(3.16), \ldots$, (3.17) até a convergência.

(iii) Obtidas as estimativas para $\boldsymbol{\beta}$ e $\gamma_{l}$ no passo anterior, executar o processo iterativo descrito em (3.18) até a convergência.

(iv) Repetir o passo 2 e o passo 3 até obter a convergência global, de forma que os resultados obtidos do passo $(i v)^{(a)}$ sejam praticamente iguais aos obtidos no passo $(i v)^{(a-1)}$.

\subsubsection{Graus de liberdade efetivos}

Similarmente aos MLPGs, os graus de liberdade efetivos $d f(\boldsymbol{\lambda})$ são definidos para os MLPAGs por meio da predição do preditor linear

$$
\hat{\boldsymbol{\eta}}=\mathbf{X} \hat{\boldsymbol{\beta}}+\mathbf{B}_{1} \hat{\gamma}_{1}+\ldots+\mathbf{B}_{r} \hat{\gamma}_{r}=\widehat{\mathbf{H}}(\boldsymbol{\lambda}) \hat{\mathbf{z}}
$$

em que (ver demonstração do Apêndice C)

$$
\mathbf{H}(\boldsymbol{\lambda})=\left[\begin{array}{ll}
\mathbf{X} & \mathbf{B}
\end{array}\right]\left[\begin{array}{cc}
\mathbf{X}^{\top} \mathbf{W} \mathbf{X} & \mathbf{X}^{\top} \mathbf{W B} \\
\mathbf{B}^{\top} \mathbf{W} \mathbf{X} & \mathbf{B}^{\top} \mathbf{W B}+\mathbf{P}(\boldsymbol{\lambda})
\end{array}\right]^{-1}\left[\begin{array}{c}
\mathbf{X}^{\top} \\
\mathbf{B}^{\top}
\end{array}\right] \mathbf{W}
$$

$\operatorname{com} \mathbf{B}=\left[\mathbf{B}_{1}, \ldots, \mathbf{B}_{r}\right]$ e

$$
\mathbf{P}(\boldsymbol{\lambda})=\text { blocodiag }\left\{\lambda_{1} \mathbf{P}_{1}^{d}, \ldots, \lambda_{r} \mathbf{P}_{r}^{d}\right\}
$$

Conforme observado por Vanegas e Paula (2016) a matriz $\left[\mathbf{X}, \mathbf{B}_{1}, \ldots, \mathbf{B}_{r}\right]$ pode ser singular, gerando problemas de identificação dos parâmetros em $\left(\boldsymbol{\beta}^{\top}, \boldsymbol{\gamma}^{\top}\right)^{\top}$. Uma sugestão (vide Wood 2017, sec. 5.4.1) é aplicar algum tipo de reparametrização na forma $\gamma_{l}=\mathbf{C}_{l} \boldsymbol{\tau}_{l}$, para $l=1, \ldots, r$, em que $\mathbf{C}_{l}$ é uma matriz de dimensão $q_{l} \times\left(q_{l}-1\right)$ obtida por meio da decomposição QR. Em particular, quando $r=1$, uma sugestão é omitir o intercepto do componente sistemático do preditor linear. 
Segundo Hastie e Tibshirani 1990 (cap. 6), os graus de liberdade efetivos na classe dos MLPAGs ficam definidos por

$$
d f(\boldsymbol{\lambda})=\operatorname{tr}\{\widehat{\mathbf{H}}(\boldsymbol{\lambda})\} .
$$

Alternativamente, para definir os graus de liberdade efetivos correspondentes ao $l$-ésimo componente não paramétrico, $\operatorname{com} l=1, \ldots, r$, alguns autores consideram a solução condicional para obter $\hat{\mathbf{f}}_{l}$ dada por

$$
\hat{\mathbf{f}}_{l}=\mathbf{B}_{l} \hat{\gamma}_{l}=\mathbf{B}_{l}\left(\mathbf{B}_{l}^{\top} \widehat{\mathbf{W}} \mathbf{B}_{l}+\lambda_{l} \mathbf{P}_{l}^{d}\right)^{-1} \mathbf{B}_{l}^{\top} \widehat{\mathbf{W}}\left(\hat{\mathbf{z}}-\mathbf{X} \hat{\boldsymbol{\beta}}-\sum_{j=1, j \neq l}^{r} \mathbf{B}_{j} \hat{\gamma}_{j}\right) .
$$

Ou seja, $d f_{\gamma_{l}}(\boldsymbol{\lambda})=\operatorname{tr}\left\{\widehat{\mathbf{H}}_{\gamma_{l}}(\boldsymbol{\lambda})\right\}$, em que

$$
\widehat{\mathbf{H}}_{\gamma_{l}}(\boldsymbol{\lambda})=\mathbf{B}_{l}\left(\mathbf{B}_{l}^{\top} \widehat{\mathbf{W}} \mathbf{B}_{l}+\lambda_{l} \mathbf{P}_{l}^{d}\right)^{-1} \mathbf{B}_{l}^{\top} \widehat{\mathbf{W}}
$$

Para fins computacionais é usual calcular $d f_{\gamma_{l}}(\boldsymbol{\lambda})$ da seguinte maneira:

$$
d f_{\gamma_{l}}(\boldsymbol{\lambda})=\operatorname{tr}\left\{\left(\mathbf{B}_{l}^{\top} \widehat{\mathbf{W}} \mathbf{B}_{l}+\lambda_{l} \mathbf{P}_{l}^{d}\right)^{-1} \mathbf{B}_{l}^{\top} \widehat{\mathbf{W}} \mathbf{B}_{l}\right\} .
$$

Assim como nos MLPGs, podemos reescrever o $\operatorname{tr}\left\{\widehat{\mathbf{H}}_{\gamma_{l}}(\boldsymbol{\lambda})\right\}$ como

$$
\begin{aligned}
d f_{\gamma_{l}}(\boldsymbol{\lambda}) & =\operatorname{tr}\left\{\widehat{\mathbf{H}}_{\gamma_{l}}(\boldsymbol{\lambda})\right\} \\
& =\operatorname{tr}\left\{\mathbf{B}_{l}^{\top} \widehat{\mathbf{W}} \mathbf{B}_{l}\left(\mathbf{B}_{l}^{\top} \widehat{\mathbf{W}} \mathbf{B}_{l}+\lambda_{l} \mathbf{P}_{l}^{d}\right)^{-1}\right\} \\
& =\operatorname{tr}\left\{\left(\mathbf{I}_{l}+\lambda_{l} \mathbf{Q}_{l}^{-\frac{1}{2}} \mathbf{P}_{l}^{d} \mathbf{Q}_{l}^{-\frac{1}{2}}\right)^{-1}\right\} \\
& =\sum_{j=1}^{q_{l}} \frac{1}{1+\lambda_{l} \alpha_{j}}
\end{aligned}
$$

em que $\alpha_{j}$ são os autovalores da matriz não negativa definida $\mathbf{Q}_{l}^{-\frac{1}{2}} \mathbf{P}_{l}^{d} \mathbf{Q}_{l}^{-\frac{1}{2}}$ e $\mathbf{Q}_{l}^{\frac{1}{2}}$ é uma matriz positiva definida, de forma que $\mathbf{B}_{l}^{\top} \widehat{\mathbf{W}} \mathbf{B}_{l}=\mathbf{Q}_{l}^{\frac{1}{2}} \mathbf{Q}_{l}^{\frac{1}{2}}$.

Nesse caso, o total de graus de liberdade efetivos fica aproximadamente dado por

$$
d f(\boldsymbol{\lambda}) \cong p+\sum_{l=1}^{r} d f_{\gamma_{l}}(\boldsymbol{\lambda})
$$

\subsubsection{Estimação do parâmetro de suavização}

Para obter as estimativas dos parâmetros $\boldsymbol{\beta}, \boldsymbol{\gamma}_{1}, \ldots, \boldsymbol{\gamma}_{r}$ do modelo prosposto na Seção 3.3, consideramos o vetor de parâmetros $\boldsymbol{\lambda}=\left(\lambda_{1}, \ldots, \lambda_{r}\right)^{\top}$ fixo.

Assim, como dito anteriormente, os parâmetros de suavização precisam ser escolhidos cuidadosamente para que se obtenha o melhor ajuste, de forma que o modelo não fique muito suave, nem interpole com os dados.

Para obter uma estimativa para esses parâmetros de suavização pode-se utilizar a validação cruzada ou a validação cruzada generalizada, assim como foi feito nos MLPGs.

Os métodos de validação cruzada e validação cruzada generalizada ficam expressos na classe dos MLPAGs da seguinte maneira: 


$$
\operatorname{VC}(\boldsymbol{\lambda})=\frac{1}{n} \sum_{i=1}^{n} \frac{\hat{w}_{i}\left(\hat{z}_{i}-x_{i}^{\top} \hat{\beta}\right)^{2}}{\left\{1-\hat{h}_{i i}(\boldsymbol{\lambda})\right\}^{2}}
$$

e

$$
\operatorname{VCG}(\boldsymbol{\lambda})=\sum_{i=1}^{n} \frac{\hat{w}_{i}\left(\hat{z}_{i}-x_{i}^{\top} \hat{\beta}\right)^{2}}{\left[1-n^{-1} \operatorname{tr}\{\hat{\mathbf{H}}(\boldsymbol{\lambda})\}\right]^{2}},
$$

em que $\hat{h}_{i i}(\boldsymbol{\lambda})$ denota o $i$-ésimo elemento da diagonal principal de $\widehat{\mathbf{H}}(\boldsymbol{\lambda})$.

$O$ vetor $\boldsymbol{\lambda}$ ótimo é aquele que minimiza essas quantidades.

Alguns pesquisadores usam $\operatorname{AIC}(\boldsymbol{\lambda})$ e $\operatorname{BIC}(\boldsymbol{\lambda})$ para selecionar $\left(\lambda_{1}, \ldots, \lambda_{r}\right)^{\top}$, bem como para obter um submodelo. Para os MLPAGs, essas quantidades são definidas, respectivamente, por

$$
\operatorname{AIC}(\boldsymbol{\lambda})=-2 L_{p}(\hat{\boldsymbol{\theta}}, \boldsymbol{\lambda})+2\{1+d f(\boldsymbol{\lambda})\}
$$

e

$$
\operatorname{BIC}(\boldsymbol{\lambda})=-2 L_{p}(\hat{\boldsymbol{\theta}}, \boldsymbol{\lambda})+\log (n)\{1+d f(\boldsymbol{\lambda})\} .
$$

Como foi mencionado no capítulo anterior, escolhas alternativas para seleção de modelos podem ser feitas com a medida $\operatorname{GAIC}(\boldsymbol{\lambda})=-2 L_{p}(\hat{\boldsymbol{\theta}}, \boldsymbol{\lambda})+k\{1+d f(\boldsymbol{\lambda})\}$, para $k=2, \ldots, \log (n)$.

\subsection{Teste de hipóteses}

Assim como nos MLPGs, suponha que inicialmente o interesse é testar uma hipótese na forma $H_{0}: \mathbf{C} \boldsymbol{\beta}=0$ contra $H_{1}: \mathbf{C} \boldsymbol{\beta} \neq 0$, em que $\mathbf{C}$ é uma matriz $k \times p \operatorname{com} k \leq p$. Sejam $\hat{\boldsymbol{\theta}}^{0}$ e $\hat{\boldsymbol{\theta}}$ as estimativas de máxima verossimilhança penalizadas sob, respectivamente, $H_{0}$ e $H_{1}$. A estatística da razão de verossimilhanças é dada por

$$
R V=2\left\{L_{p}\left(\hat{\boldsymbol{\theta}}, \boldsymbol{\lambda}_{1}\right)-L_{p}\left(\hat{\boldsymbol{\theta}}^{0}, \boldsymbol{\lambda}_{0}\right)\right\},
$$

e segue, sob $H_{0}$, uma distribuição qui-quadrado com graus de liberdade dados por $d f\left(\boldsymbol{\lambda}_{1}\right)-d f\left(\boldsymbol{\lambda}_{0}\right)$, em que $\boldsymbol{\lambda}=\left(\lambda_{1}, \ldots, \lambda_{r}\right)^{\top}$.

Conforme definido na Seção 3.1, sabemos que para os MLPAGs a função não paramétrica é dada por $f_{l}\left(t_{i l}\right)=\sum_{j=1}^{q_{l}} B_{j l}^{\left(m_{l}\right)}\left(t_{i l}\right) \gamma_{j l}=\sum_{l=1}^{r} \mathbf{B}_{l} \gamma_{l}$. Sendo assim, vamos testar as hipóteses $H_{0}: \gamma_{l}=0$ contra $H_{1}: \gamma_{l} \neq 0$ para saber se a $l$-ésima variável explicativa tratada de forma não paramétrica é significativa. Considerando a matriz de variância-covariância definida por $\mathbf{V}_{\boldsymbol{\theta} \boldsymbol{\theta}}=$ $\left[\mathbf{K}_{p}^{\boldsymbol{\theta} \boldsymbol{\theta}}(\boldsymbol{\theta})\right]^{-1} \mathbf{K}_{\boldsymbol{\theta} \boldsymbol{\theta}}(\boldsymbol{\theta})\left[\mathbf{K}_{p}^{\boldsymbol{\theta} \boldsymbol{\theta}}(\boldsymbol{\theta})\right]^{-1}, \operatorname{com} \boldsymbol{\theta}=\left(\boldsymbol{\beta}^{\top}, \boldsymbol{\gamma}_{1}^{\top}, \ldots, \boldsymbol{\gamma}_{r}^{\top}, \phi\right)^{\top}$, segundo Vanegas e Paula (2016), temos que o efeito da $l$-ésima função não paramétrica é insignificante quando a estatística apresentada abaixo assume valores pequenos

$$
W_{\gamma_{l}}=\hat{\gamma}_{l}^{\top} \widehat{\operatorname{Var}}^{-1}\left[\hat{\gamma}_{l}\right] \hat{\gamma}_{l},
$$

sendo que, sob a hipótese nula, $W_{\gamma_{l}}$ segue assintoticamente uma distribuição $\chi_{q_{l}}^{2}$, para $l=1, \ldots, r$. Da mesma forma que foi observado no Capítulo 2, a penalização leva a uma redução do espaço paramétrico $\gamma_{l}$ e, como consequência, os graus de liberdade referentes a essa estatística de Wald 
devem ser na prática muito menores do que $q_{l}$. Sendo assim, utilizar $q_{l}$ pode levar a uma perda do poder e, por isso, Wood (2017) sugere utilizar $d f_{\gamma_{l}}(\boldsymbol{\lambda})$ ao invés de $q_{l}$.

\subsection{Considerações}

Este capítulo teve como principal objetivo estudar a teoria dos modelos lineares parciais aditivos generalizados como uma extensão do modelo apresentado no Capítulo 2. Sendo assim, definimos o modelo a ser estudado, propusemos novamente o uso do método de suavização P-splines para estimar os componentes não paramétricos do modelo, apresentamos o logaritmo da função de verossimilhança penalizada, as funções escore penalizadas e a matriz de informação de Fisher penalizada. Somadas à isso, apresentamos a estimação dos parâmetros paramétricos e não paramétricos do modelo pela combinação do processo iterativo de Fisher e o algoritmo backfitting, discutimos a derivação dos graus de liberdade efetivos, apresentamos o método para estimar o vetor de parâmetros de suavização a partir dos métodos de validação cruzada, validação cruzada generalizada e medidas de informação e por fim, discutimos teste de hipóteses. 


\section{Capítulo 4}

\section{Aplicações}

Neste capítulo, iremos reanalisar os três exemplos descritos no Capítulo $1 \mathrm{com}$ a finalidade de ilustrar a teoria apresentada neste trabalho. Para isso, será utilizado a biblioteca gamlss que foi implementado por Stasinopoulos e Ribgy (2007). Para mais detalhes sobre a teoria do GAMLSS, ver também Ribgy e Stasinopoulos (2005).

\subsection{Garotas de Varsóvia}

Iniciamos aplicando a teoria apresentada para analisar o exemplo das Garotas de Varsóvia, descrito na Seção 1.2.1. Como vimos anteriormente, o comportamento da proporção de garotas que já menstruaram conforme a idade é não linear. Por conta disso, propusemos o modelo (1.3), apresentado novamente a seguir

$$
\begin{gathered}
y_{i} \mid \text { idade }_{i} \stackrel{i n d}{\sim} B\left(m_{i}, \pi_{i}\right) \text { com } \\
\log \left\{\frac{\pi_{i}}{1-\pi_{i}}\right\}=\alpha+f\left(\text { idade }_{i}\right) .
\end{gathered}
$$

Ajustando o modelo proposto, obtemos as estimativas de máxima verossimilhança penalizadas apresentadas na Tabela 4.1. Nesse caso, a função $f($.) foi ajustada por meio dos P-splines, adotando um B-spline cúbico, a penalização de diferenças quadrática, 21 nós equidistantes e utilizando o critério de informação de Akaike generalizado (GAIC) para a estimação do parâmetro de suavização $\lambda$. Um ponto de atenção ao analisar a estimativa fornecida na Tabela 4.1 para o termo de suavização P-spline(Idade), decomposto em uma parte linear e uma não linear, é que a mesma se refere apenas ao componente linear da função de suavização (função não paramétrica) e não à sua contribuição total. Isso porque o gamlss ajusta o componente linear do termo de suavização juntamente com as outras variáveis tratadas de forma paramétrica no modelo. A contribuição total da função de suavização é obtida por meio do gráfico apresentado na Figura 4.1. Com relação aos graus de liberdade efetivos, por definição, temos que $d f(\lambda)=1+d f_{\gamma}$ (idade), em que 1 se refere ao intercepto.

Assim, como era esperado na análise descritiva dos dados, vemos pela Figura 4.1 que à medida que aumenta a idade, espera-se um aumento na probabilidade de ocorrência do início do período de menstruação, ou seja, para idades mais elevadas é esperado que a proporção de garotas que já menstruaram seja maior. Além disso, nota-se que a banda de confiança pontual de $95 \%$ indica maior variabilidade para a proporção de garotas que já menstruaram que possuem idade mais baixa 
Tabela 4.1: Estimativas de máxima verossimilhança penalizadas referentes ao modelo ajustado, seus respectivos erros padrão aproximados e valores-p, aos dados Garotas de Varsóvia

\begin{tabular}{lrrr}
\hline Efeito & Estimativa & Erro padrão & Valor-p \\
\hline Intercepto & $-18,54$ & 0,81 & 0,00 \\
\hline \hline P-spline(Idade) & 1,41 & & 0,00 \\
$d f(\lambda)$ & 4,44 & & \\
$d f_{\gamma}($ idade) & 3,44 & & \\
\hline \hline
\end{tabular}

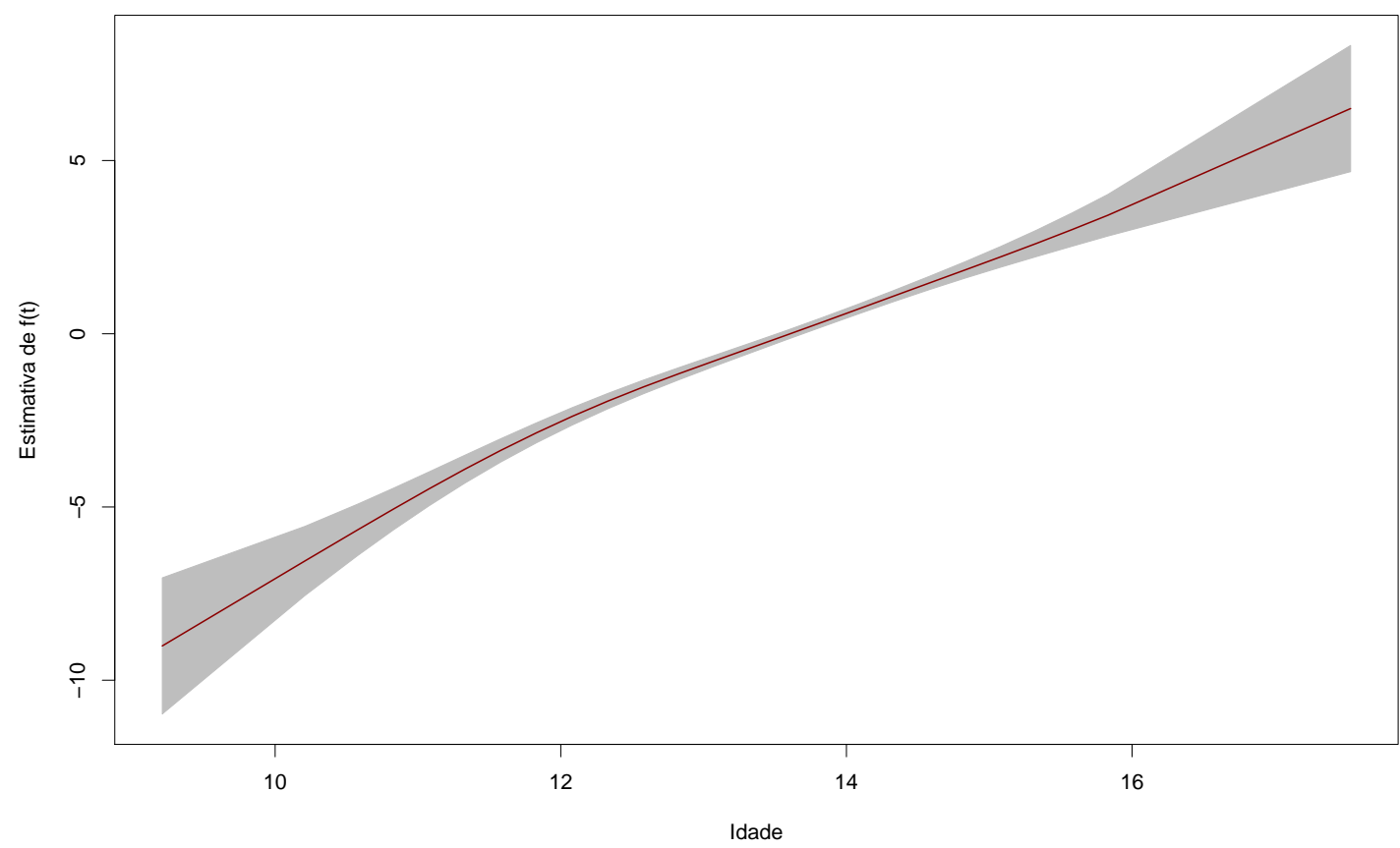

Figura 4.1: Banda de confiança pontual de $95 \%$ para a função $f(t)$ para os dados Garotas de Varsóvia. 


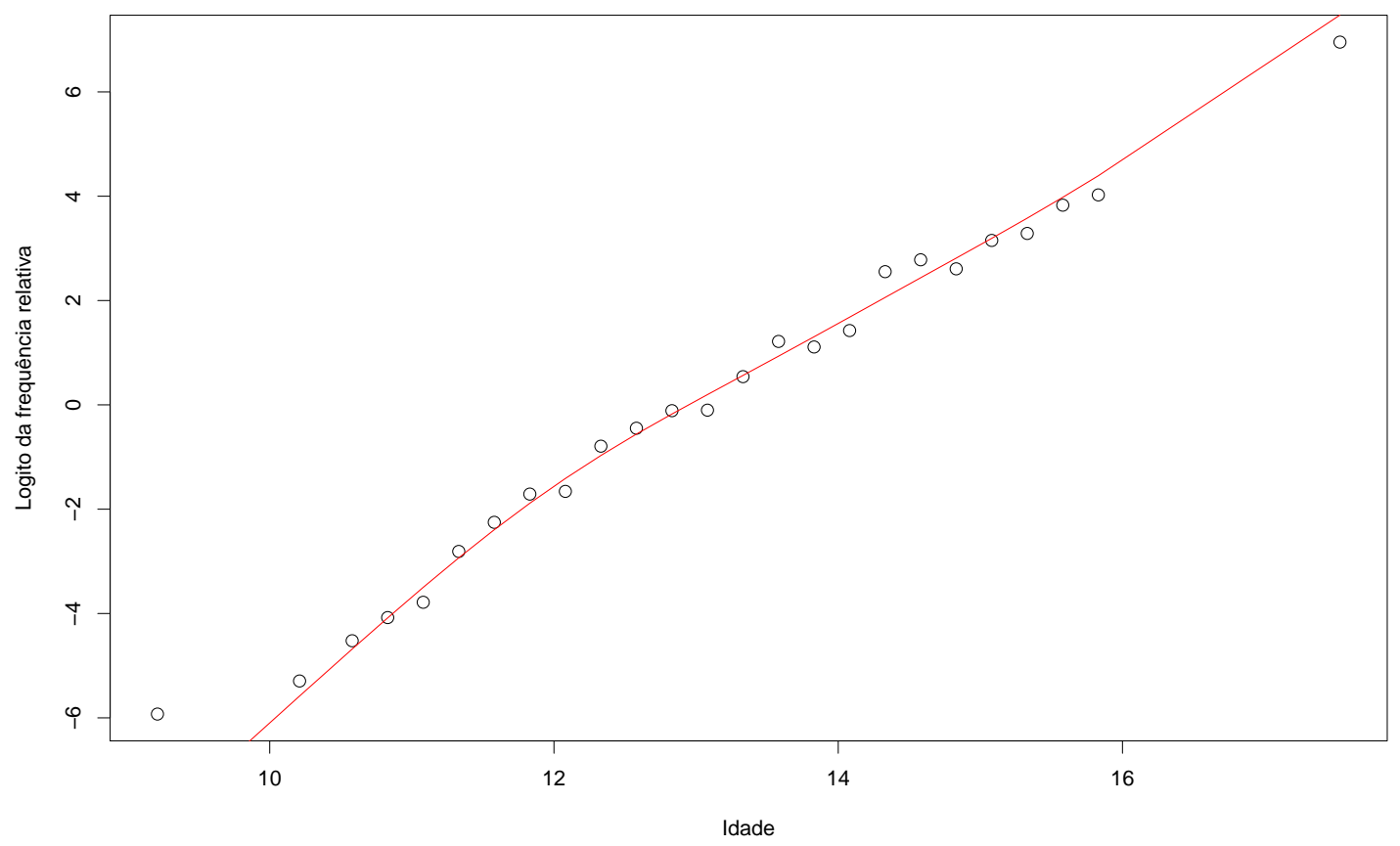

Figura 4.2: Curva ajustada para o logito da proporção de meninas com menstruação já iniciada.

e mais alta. Isso pode ser explicado pelo fato de que apesar de não ser comum, é possível que garotas muito novas já tenham menstruado, assim como garotas mais velhas ainda não tenham menstruado, fazendo com que as extremidades sejam mais difíceis de prever.

Vale ressaltar que todas as bandas de confiança apresentadas neste trabalho, feitas pelo gamlss, são construídas ponto a ponto, ou seja, os intervalos de confiança de $95 \%$ são construídos para cada valor da variável explicativa separadamente. Sendo assim, a probabilidade de $95 \%$ é válida apenas para cada ponto e não simultaneamente para toda a função.

A curva obtida para os dados a partir do modelo proposto é apresentada na Figura 4.2. Nota-se que a curva está bem ajustada aos dados.

\subsubsection{Diagnóstico}

Para avaliar se o ajuste obtido para o modelo proposto está adequado, na Figura 4.3 são apresentados os gráficos dos resíduos do modelo. Essa análise de diagnóstico é feita pelo gamlss.

É possível observar que o gráfico de resíduos quantílicos contra o valor ajustado apresenta comportamento aleatório em torno de zero, o que indica que a variabilidade dos resíduos é constante. O gráfico de resíduos quantílicos contra o índice também apresenta comportamento aleatório, indicando a independência dos resíduos. Além disso, a estimativa de densidade dos resíduos possui um comportamente aproximadamente normal, da mesma forma que no gráfico Q-Q plot, o comportamento é praticamente linear.

Dessa forma, os gráficos sugerem que os resíduos seguem aproximadamente uma distribuição Normal, $r_{q_{i j}} \sim N(0,1)$ e que o modelo está bem ajustado aos dados. 

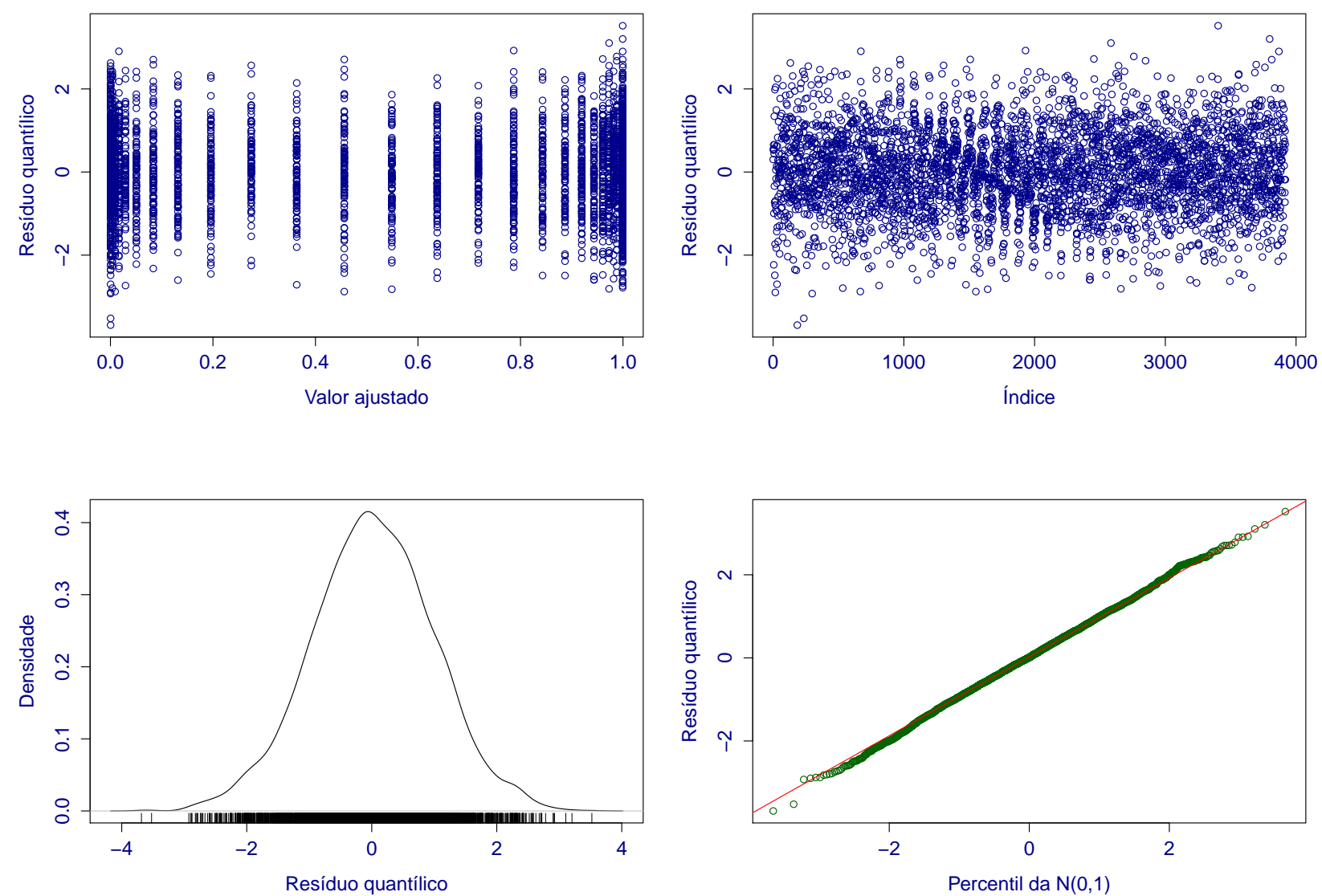

Figura 4.3: Gráficos de resíduos referentes ao modelo ajustado aos dados Garotas de Varsóvia. 
Tabela 4.2: Estimativas de máxima verossimilhança penalizadas referentes ao modelo ajustado, seus respectivos erros padrão aproximados e valores-p, aos dados Clientes de uma Loja.

\begin{tabular}{lrrr}
\hline Efeito & Estimativa & Erro padrão & Valor-p \\
\hline Intercepto & 2,94 & 0,21 & 0,00 \\
Renda & $-1,1 \times 10^{-5}$ & $2,1 \times 10^{-6}$ & 0,00 \\
Idade & $-3,5 \times 10^{-3}$ & $1,8 \times 10^{-3}$ & 0,05 \\
\hline \hline P-spline(domic) & $5,9 \times 10^{-4}$ & & 0,00 \\
P-spline(dist1) & 0,16 & & 0,00 \\
P-spline(dist2) & $-0,13$ & & 0,00 \\
$d f(\boldsymbol{\lambda})$ & 7,95 & & \\
$d f_{\gamma}($ domic $)$ & 1,00 & & \\
$d f_{\gamma}($ dist1) & 2,95 & & \\
$d f_{\gamma}$ (dist2) & 1,00 & & \\
\hline \hline
\end{tabular}

\subsection{Clientes de uma loja}

Vamos retornar ao segundo exemplo motivacional apresentado na Seção 1.2.2.

Como vimos na análise descritiva dos dados, o número de clientes aparenta possuir comportamento não linear quando avaliado nas variáveis domicílio, distância ao concorrente mais próximo e distância à loja. Sendo assim, o modelo (1.4) foi proposto:

$$
\begin{gathered}
y_{i} \mid \mathbf{x}_{i} \stackrel{i n d}{\sim} P\left(\mu_{i}\right) \text { com } \\
\log \left(\mu_{i}\right)=\beta_{0}+\beta_{1} \text { renda }_{i}+\beta_{2} \text { idade }_{i}+f_{1}\left(\text { domic }_{i}\right)+f_{2}\left(\text { dist }_{i}\right)+f_{3}\left(\text { dist }_{i}\right) .
\end{gathered}
$$

As variáveis de comportamento não paramétrico foram ajustadas por meio de $\mathrm{P}$-splines considerando B-splines cúbicos, a penalidade de diferenças de segunda ordem e o método GAIC para estimar os parâmetros de suavização $\boldsymbol{\lambda}$. Além disso, foram adotados 20 intervalos igualmente espaçados entre o mínimo e o máximo valor da variável ajustada, ou seja, 21 nós.

A Tabela 4.2 apresenta as estimativas de máxima verossimilhança penalizadas com os respectivos erros padrão aproximados. Observa-se que todas as variáveis explicativas são significativas. Como dito na seção anterior, para as variáveis explicativas domicílio, distância ao concorrente mais próximo e distância à loja, que foram tratadas de forma não linear, as estimativas fornecidas na tabela se referem à parte linear da função de suavização e não à sua contribuição total, por isso, esses valores devem ser interpretados com cautela. Na Figura 4.4, a contribuição total dessas variáveis é obtida. Com relação aos graus de liberdade efetivos obtidos no ajuste, temos que $d f(\boldsymbol{\lambda})=3+d f_{\gamma}($ domic $)+$ $d f_{\gamma}(d i s t 1)+d f_{\gamma}(d i s t 2)$, em que 3 se refere ao intercepto e às variáveis renda e idade. Os graus de liberdade das variáveis explicativas número de domicílios e distância à loja é igual a 1. Isso nos dá indícios de que a relação do número de clientes com essas duas variáveis é linear. Dessa forma, poderíamos ter ajustado o modelo tratando as variáveis número de domicílios e distância à loja de forma paramétrica.

A Figura 4.4 apresenta as predições para os componentes paramétricos e não paramétricos do modelo com a respectiva banda de confiança pontual aproximada de $95 \%$.

Assim como esperado na análise preliminar, vemos que o número de clientes aumenta de forma 



Figura 4.4: Bandas de confiança pontuais aproximadas de $95 \%$ para os efeitos das variáveis explicativas referentes ao modelo ajustado aos dados Clientes de uma Loja.

não linear quando a distância ao concorrente mais próximo fica maior. Além disso, o número esperado de clientes aumenta quando existem mais domicílios em uma determinada área e diminui conforme a distância do domícilio à loja fica maior, porém o comportamento do valor do aluguel com relação à essas duas variáveis é linear, assim como os graus de liberdade efetivos indicaram. Para as variáveis renda e idade, vemos que o modelo indica uma diminuição do número de clientes da área quando aumenta a idade média dos domícilios e quando aumenta a renda média dos domicílios da área.

\subsubsection{Diagnóstico}

Para avaliar a adequabilidade do modelo, são apresentadas na Figura 4.5 as análises de diagnóstico do ajuste.

Observando inicialmente o gráfico de resíduos quantílicos contra o valor ajustado, vemos que os pontos se distribuem aleatoriamente em torno de zero, o que nos indica a homogeneidade da variabilidade. O segundo gráfico de resíduos quantílicos contra o índice destaca poucos pontos fora da faixa $[-2,2]$. No gráfico $Q-Q$ plot, apesar de alguns pontos estarem fora da diagonal, vemos que os resíduos possuem comportamento aproximadamente linear, além disso, no gráfico de densidade os resíduos apresentam uma forma que se aproxima da distribuição normal.

Sendo assim, podemos considerar que os resíduos possuem uma distribuição aproximadamente normal, $r_{q_{i}} \sim N(0,1)$, e, consequentemente, que o modelo está bem ajustado. 

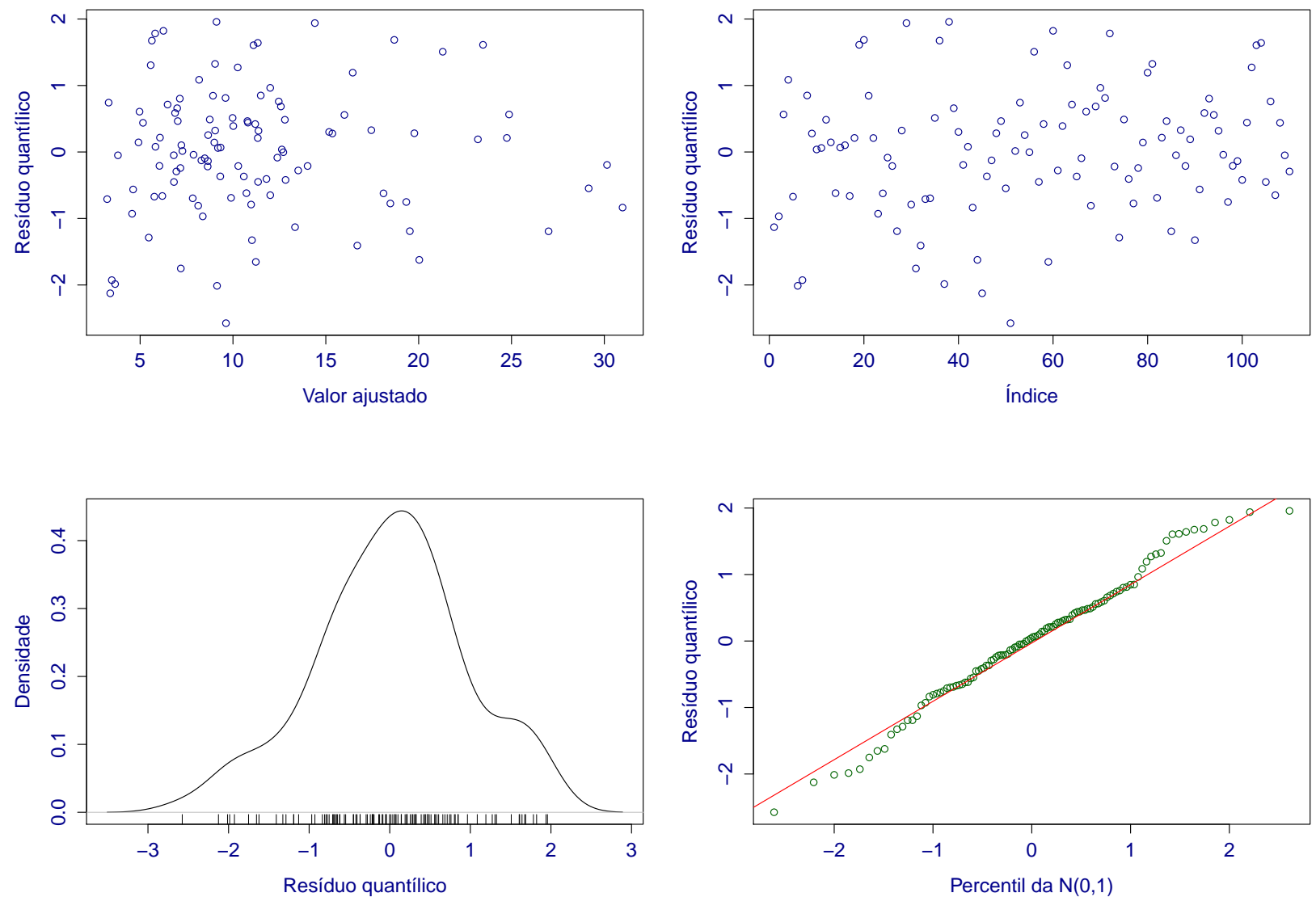

Figura 4.5: Gráficos de resíduos referentes ao modelo ajustado aos dados Clientes de uma Loja. 
Tabela 4.3: Estimativas de máxima verossimilhança penalizadas referentes ao modelo utilizando a distribuição gama ajustado, seus respectivos erros padrão aproximados e valores-p, aos dados Valor do Aluguel.

\begin{tabular}{lrrr}
\hline Efeito & Estimativa & Erro padrão & Valor-p \\
\hline Intercepto & $-2,29$ & 0,41 & 0,00 \\
localBoa & 0,10 & 0,01 & 0,00 \\
localExcelente & 0,21 & 0,03 & 0,00 \\
banhPremium & 0,07 & 0,02 & 0,00 \\
cozPremium & 0,13 & 0,03 & 0,00 \\
Com Aquec. & 0,33 & 0,02 & 0,00 \\
\hline \hline P-spline(area) & 0,01 & & 0,00 \\
P-spline(ano) & 0,00 & & 0,00 \\
$d f(\boldsymbol{\lambda})$ & 18,04 & & \\
$d f_{\gamma}$ (area) & 3,22 & & \\
$d f_{\gamma}$ (ano) & 7,82 & & \\
\hline \hline
\end{tabular}

\subsection{Valor de aluguel em Munique}

O último exemplo motivacional apresentado na Seção 1.2.3 é sobre o valor do aluguel na cidade de Munique. Conforme dito anteriormente, percebemos na análise descritiva que as varáveis área e ano de construção têm comportamento não linear. Dessa forma, propusemos o modelo (1.5), apresentado novamente a seguir, em que essas variáveis são introduzidas de forma não paramétrica,

$$
\begin{gathered}
y_{i} \mid \mathbf{x}_{i} \stackrel{i n d}{\sim} G\left(\mu_{i}, \sigma\right) \text { com } \\
\log \left(\mu_{i}\right)=\beta_{0}+\beta_{11} l_{o c} c_{1 i}+\beta_{12} l o c_{2 i}+\beta_{2} \text { banh }_{i}+\beta_{3} \text { coz }_{i}+\beta_{4} \text { aquec }_{i}+f_{1}\left(\text { area }_{i}\right)+f_{2}\left(\text { ano }_{i}\right) .
\end{gathered}
$$

Assim como nos exemplos anteriores, as funcões $f($.$) , vão ser estimadas por meio dos P-splines,$ utilizando B-splines cúbicos, a penalidade de diferenças de segunda ordem e considerando 21 nós equidistantes. Além disso, os parâmetros de suavização $\boldsymbol{\lambda}$ serão estimados por meio do método de validação cruzada generalizada.

Na Tabela 4.3 são apresentadas as estimativas de máxima verossimilhança obtidas para o modelo proposto, sendo que para as variáveis explicativas área e ano de construção que foram tratadas de forma não paramétrica, as estimativas se referem apenas ao componente linear do termo de suavização. A contribuição total da função de suavização é obtida na Figura 4.6. Com relação aos graus de liberdade efetivos, por definição, temos que $d f(\boldsymbol{\lambda})=6+1+d f_{\gamma}($ area $)+d f_{\gamma}($ ano $)$, em que 6 se refere ao intercepto e às variáveis tratadas de forma linear e 1 se refere ao coeficiente de variação $\sigma$.

A Figura 4.6 mostra as funções suavizadas ajustadas do modelo semiparamétrico. Vemos que o valor médio do aluguel cresce de forma não linear quando o tamanho do apartamento aumenta. Para a variável explicativa ano de construção, assim como foi indicado na análise descritiva, o valor médio do aluguel de apartamentos construídos a partir de 1960 fica mais caro à medida que a construção é mais recente, de forma que antes desse ano, o valor do aluguel varia pouco. Para os fatores, nota-se pela Tabela 4.3 que quanto melhor a localização do apartamento, mais caro é o aluguel cobrado, assim como instalações melhores de banheiro e cozinha aumentam o preço do aluguel. Com relação ao aquecimento, claramente os apartamentos que possuem aquecimento central possuem valor do 

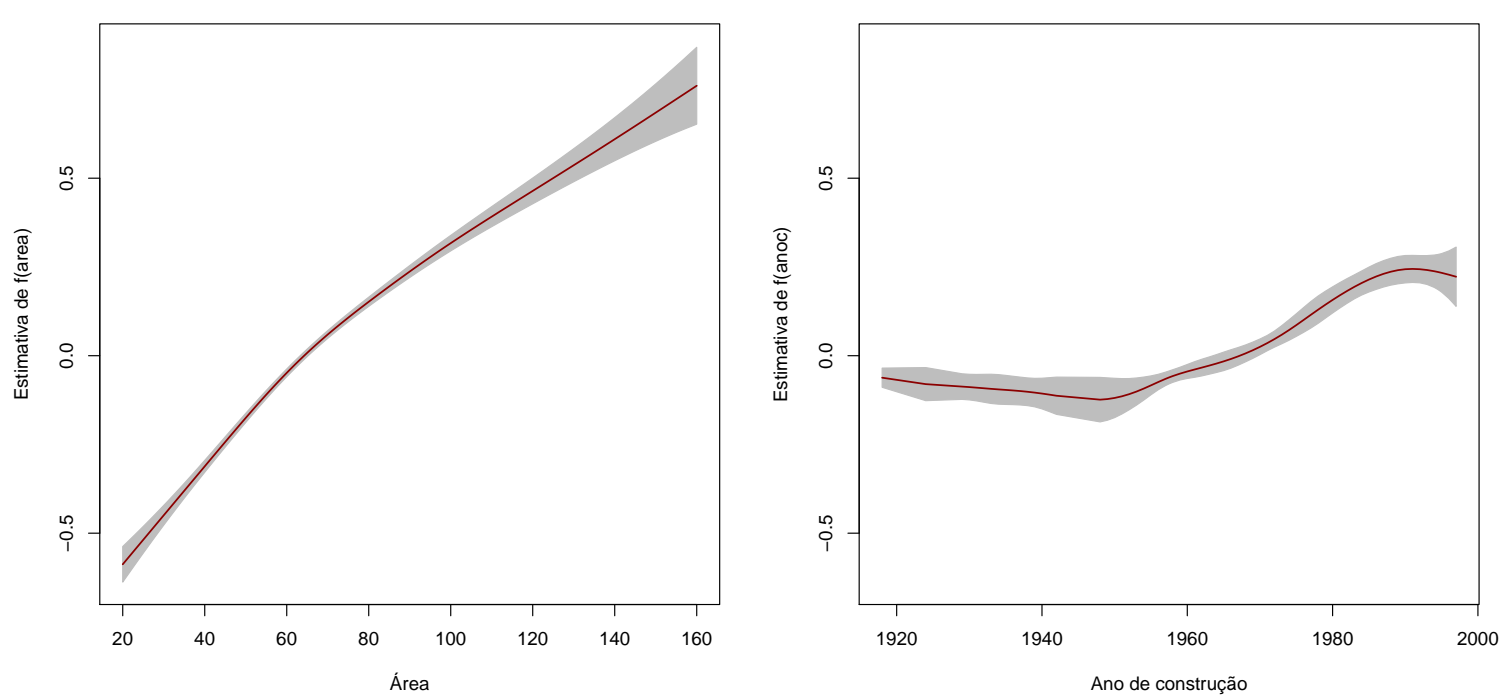

Figura 4.6: Bandas de confiança pontuais aproximadas de $95 \%$ para os efeitos das variáveis explicativas área e ano de construção no valor médio do aluguel referentes ao modelo utilizando a distribuição gama ajustado aos dados Valor do Aluguel.

aluguel mais caro.

Para avaliar se o modelo está bem ajustado, são apresentados os gráficos de resíduos da análise de diagnóstico feito pelo gamlss na Figura 4.7. Nota-se pelo gráfico normal de probabilidades (Q-Q plot) com o resíduo quantílico um certo afastamento da normalidade, sendo indicado pelo gráfico de densidade uma assimetria à esquerda para a distribuição dos resíduos. Assim, uma outra distribuição assimétrica, por exemplo, uma gama com três parâmetros, poderia melhorar a qualidade do ajuste. Notamos também pelo gráfico do resíduo quantílico contra o valor ajustado que a variabilidade não foi totalmente controlada. Embora o modelo gama seja heteroscedástico (variância não constante) estamos assumindo o coeficiente de variação $\sigma$ como constante. Uma tentativa para melhorar a qualidade do ajuste seria modelar conjuntamente a média $\mu$ e o coeficiente de variação $\sigma$ (modelagem dupla) como fazemos a seguir.

Sendo assim, vamos propor um segundo modelo para $\mu$ e $\sigma$ para ajustar os dados do valor do aluguel:

$$
y_{i} \mid \mathbf{x}_{i} \stackrel{i n d}{\sim} G\left(\mu_{i}, \sigma_{i}\right)
$$

em que

$$
\begin{aligned}
& \log \left(\mu_{i}\right)=\beta_{10}+\beta_{11} \text { loc }_{1 i}+\beta_{12} \text { loc }_{2 i}+\beta_{13} \text { banh }_{i}+\beta_{14} \text { coz }_{i}+\beta_{15} \text { aquec }_{i}+f_{1}^{\mu}\left(\text { area }_{i}\right)+f_{2}^{\mu}\left(\text { ano }_{i}\right) \text { e } \\
& \log \left(\sigma_{i}\right)=\beta_{20}+\beta_{21} l_{\text {loc }} 1 i+\beta_{22} \text { loc }_{2 i}+\beta_{23} \text { banh }_{i}+\beta_{24} \text { coz }_{i}+\beta_{25} \text { aquec }_{i}+f_{1}^{\sigma}\left(\text { area }_{i}\right)+f_{2}^{\sigma}\left(\text { ano }_{i}\right) .
\end{aligned}
$$

Utilizando o critério de seleção de modelos GAIC, obtemos o seguinte modelo final:

$$
y_{i} \mid \mathbf{x}_{i} \stackrel{i n d}{\sim} G\left(\mu_{i}, \sigma_{i}\right)
$$





Figura 4.7: Gráficos de resíduos referentes ao modelo utilizando a distribuição gama ajustado aos dados Valor do Aluguel.

em que

$$
\begin{gathered}
\log \left(\mu_{i}\right)=\beta_{10}+\beta_{11} \operatorname{loc}_{1 i}+\beta_{12} \text { loc }_{2 i}+\beta_{13} \text { banh }_{i}+\beta_{14} \text { coz }_{i}+\beta_{15} \text { aquec }_{i}+f_{1}^{\mu}\left(\text { area }_{i}\right)+f_{2}^{\mu}\left(\text { ano }_{i}\right) \text { e } \\
\log \left(\sigma_{i}\right)=\beta_{20}+\beta_{21} \text { loc }_{1 i}+\beta_{22} \text { loc }_{2 i}+\beta_{23} \text { aquec }_{i}+f_{1}^{\sigma}\left(\text { area }_{i}\right)+f_{2}^{\sigma}\left(\text { ano }_{i}\right)
\end{gathered}
$$

sendo $\mathbf{x}_{\mathbf{i}}=\left(1, \text { loc }_{1 i}, \operatorname{loc}_{2 i}, \text { banh }_{i}, \text { coz }_{i}, \text { aquec }_{i}, \text { area }_{i}, \text { ano }_{i}\right)^{\top}$.

Ou seja, para modelar o coeficiente de variação, apenas as variáveis área, ano de construção, localização e aquecimento central são significativas. Para a localização $\mu$, todas as variáveis são significativas.

Utilizando o pacote gamlss, obtemos as estimativas dos parâmetros para o modelo (4.2) apresentadas nas Tabelas 4.4 e 4.5, sendo que as estimativas apresentadas para as variáveis área e ano de construção se referem apenas à parte linear da função de suavização, não à sua contribuição total. Com relação aos graus de liberdade efetivos, temos que $d f(\boldsymbol{\lambda})=d f\left(\boldsymbol{\lambda}_{\mu}\right)+d f\left(\boldsymbol{\lambda}_{\sigma}\right)=$ $\left[6+d f_{\gamma}\left(\right.\right.$ area $\left._{\mu}\right)+d f_{\gamma}\left(\right.$ ano $\left.\left._{\mu}\right)\right]+\left[4+d f_{\gamma}\left(\right.\right.$ area $\left._{\sigma}\right)+d f_{\gamma}\left(\right.$ ano $\left.\left._{\sigma}\right)\right]=24,65$. Os graus de liberdade das variáveis área e ano de construção referentes ao ajuste do coeficiente de variação são próximos de 1. Isso nos dá indícios de que a relação dessas variáveis com o $\sigma$ é linear.

A Figura 4.8 mostra o ajuste obtido para o modelo proposto para $\mu$. Assim como ocorreu no modelo anterior, nota-se que no modelo proposto para $\mu$, quanto maior a área do apartamento e quanto melhor a localização do imóvel, mais alta é a média do valor do aluguel cobrado. Além disso, apartamentos com instalações de banheiro e cozinha melhores e que possuem aquecimento também 
Tabela 4.4: Estimativas de máxima verossimilhança penalizadas para os efeitos da localização referentes ao modelo duplo utilizando a distribuição gama ajustado, seus respectivos erros padrão aproximados e valores-p, aos dados Valor do Aluguel.

\begin{tabular}{lrrr}
\hline Efeito & Estimativa & Erro padrão & Valor-p \\
\hline Intercepto & $-2,32$ & 0,40 & 0,00 \\
localBoa & 0,09 & 0,01 & 0,00 \\
localExcelente & 0,20 & 0,04 & 0,00 \\
banhPremium & 0,06 & 0,02 & 0,01 \\
cozPremium & 0,10 & 0,02 & 0,00 \\
Com Aquec. & 0,32 & 0,02 & 0,00 \\
\hline \hline P-spline(area) & 0,01 & & 0,00 \\
P-spline(ano) & 0,00 & & 0,00 \\
$d f(\boldsymbol{\lambda})$ & 24,65 & & \\
$d f\left(\boldsymbol{\lambda}_{\mu}\right)$ & 18,64 & & \\
$d f_{\gamma}\left(\right.$ area $\left._{\mu}\right)$ & 5,05 & & \\
$d f_{\gamma}\left(\right.$ ano $\left._{\mu}\right)$ & 7,58 & & \\
\hline \hline
\end{tabular}

Tabela 4.5: Estimativas de máxima verossimilhança penalizadas para os efeitos do coeficiente de variação referentes ao modelo duplo utilizando a distribuição gama ajustado, seus respectivos erros padrão aproximados e valores-p, aos dados Valor do Aluguel.

\begin{tabular}{lrrr}
\hline Efeito & Estimativa & Erro padrão & Valor-p \\
\hline Intercepto & 9,78 & 0,67 & 0,00 \\
localBoa & 0,06 & 0,03 & 0,01 \\
localExcelente & 0,26 & 0,08 & 0,00 \\
Com Aquec. & $-0,23$ & 0,04 & 0,00 \\
\hline \hline P-spline (area) & 0,00 & & 0,03 \\
P-spline (ano) & $-0,01$ & & 0,00 \\
$d f(\boldsymbol{\lambda})$ & 24,65 & & \\
$d f\left(\boldsymbol{\lambda}_{\sigma}\right)$ & 6,01 & & \\
$d f_{\gamma}\left(\right.$ area $\left._{\sigma}\right)$ & 1,00 & & \\
$d f_{\gamma}\left(\right.$ ano $\left._{\sigma}\right)$ & 1,01 & & \\
\hline \hline
\end{tabular}

são mais caros em média. Para a variável ano de construção, vemos que a partir de 1960, quanto mais nova for a construção, mais caro é o valor do aluguel cobrado, sendo que antes desse ano de construção, o valor do aluguel é praticamente constante.

Na Figura 4.9 são mostrados os ajustes das funções suavizadas obtidas para o modelo proposto para $\sigma$. Como a análise descritiva já havia indicado, vemos que quanto maior a área, maior é o coeficiente de variação do valor do aluguel e quanto mais recente o ano de construção do imóvel, menor é o coeficiente de variação do valor do aluguel. Porém, assim como os graus de liberdade efetivos já indicaram, é possível notar pelos gráficos que a relação dessas duas variáveis com o valor do aluguel é linear. Além disso, para a variável localização do apartamento, vemos que quanto melhor a localização, maior é o coeficiente de variação do aluguel cobrado. Já, com relação ao aquecimento central, vemos que há menor coeficiente de variação do valor de aluguel quando o apartamento possui aquecimento. 

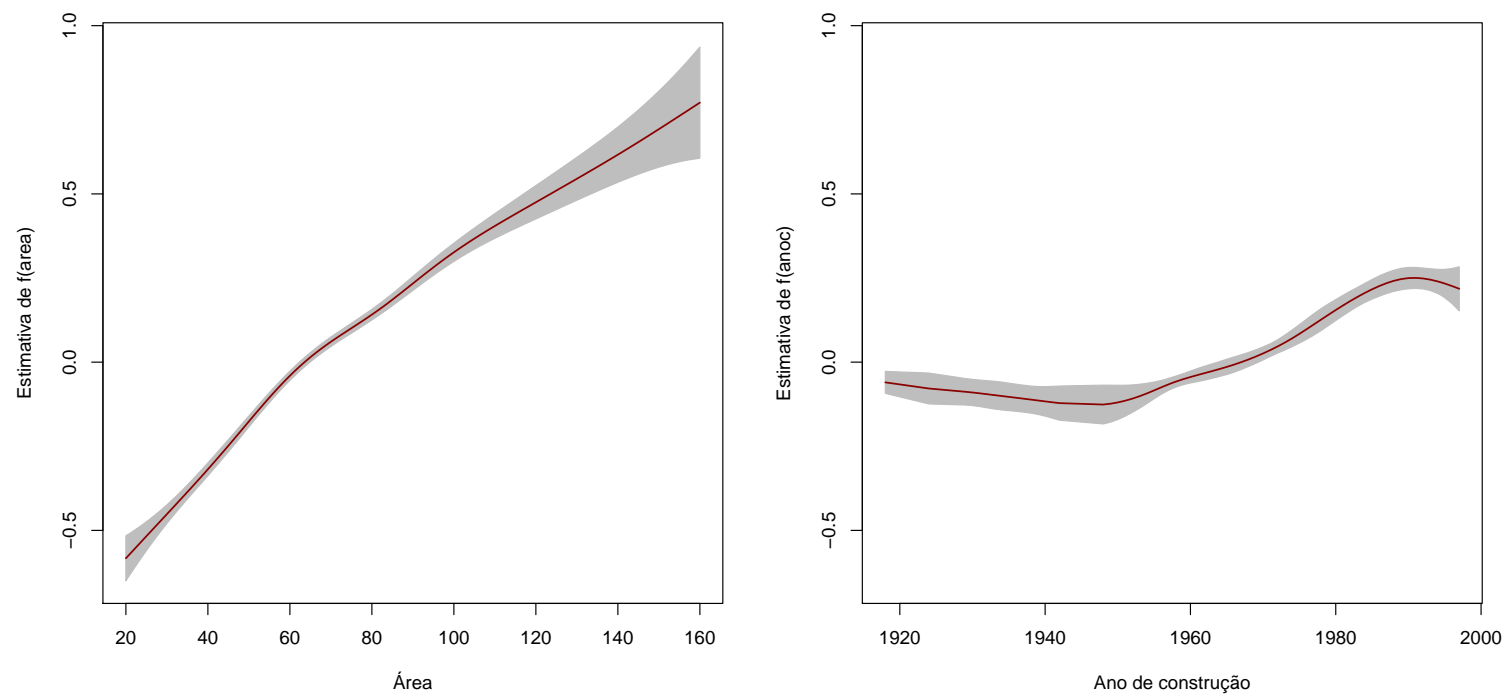

Figura 4.8: Bandas de confiança pontuais aproximadas de $95 \%$ para os efeitos das variáveis explicativas área e ano de construção no valor médio do aluguel referentes ao modelo duplo utilizando a distribuição gama ajustado aos dados Valor do Aluguel.
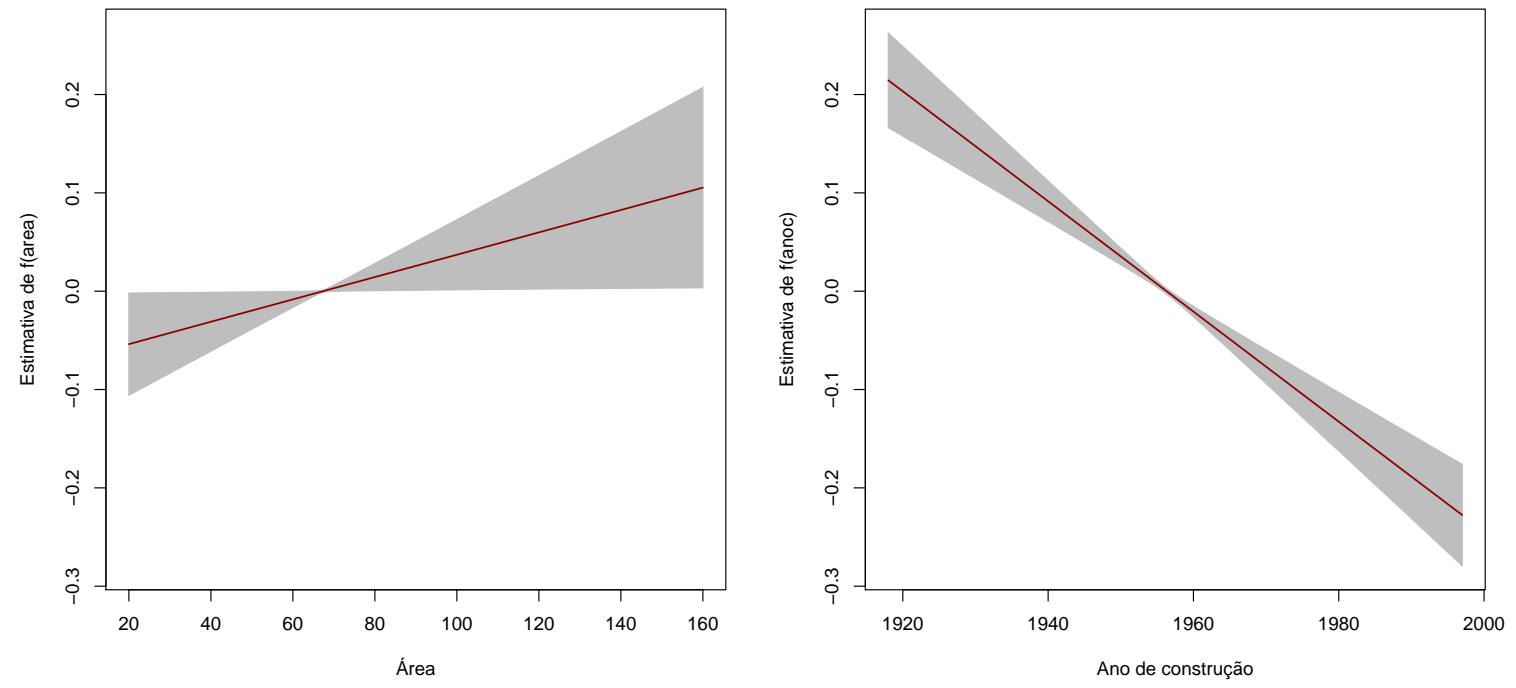

Figura 4.9: Bandas de confiança pontuais aproximadas de $95 \%$ para os efeitos das variáveis explicativas área e ano de construção no coeficiente de variação do aluguel referentes ao modelo duplo utilizando a distribuição gama ajustado aos dados Valor do Aluguel. 

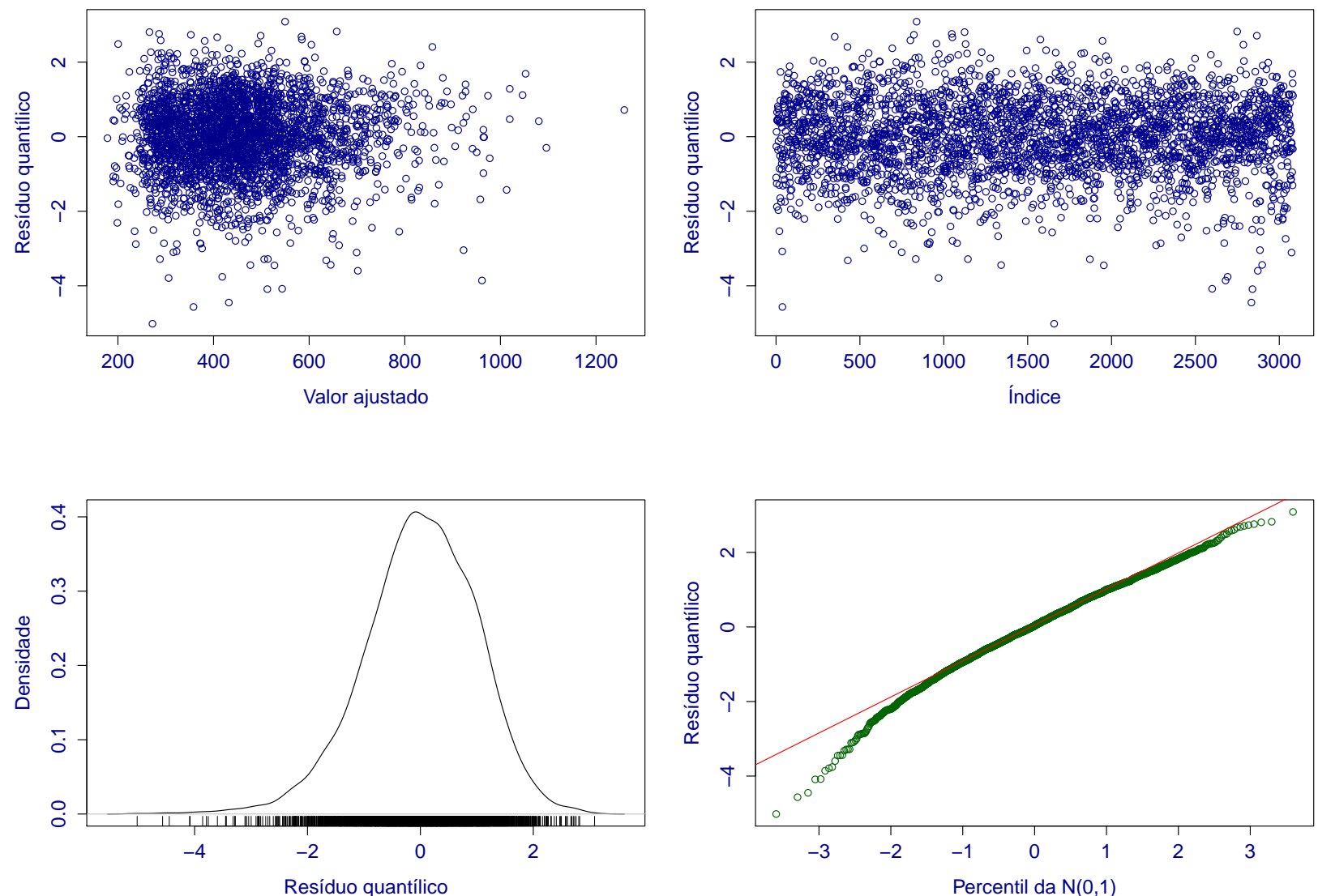

Figura 4.10: Gráficos de resíduos referentes ao modelo duplo utilizando a distribuição gama ajustado aos dados Valor do Aluguel.

Para avaliar se esse segundo modelo proposto está bem ajustado, a análise de diagnóstico fornecida pelo gamlss é apresentada na Figura 4.10.

Vemos pelo gráfico de resíduos contra o valor ajustado que os resíduos se distribuem aleatoriamente em torno de zero, indicando que para o ajuste do modelo duplo houve uma melhora no controle da variabilidade. O gráfico com a estimativa da densidade apresenta um comportamento próximo da normal, porém ainda indicando uma assimetria negativa. Por fim, vemos que no gráfico Q-Q plot os resíduos apresentam um comportamento aproximadamente linear, porém a assimetria à esquerda se manteve.

Comparando os modelos propostos para esses dados, nota-se que houve ganho no ajuste ao modelar conjuntamente $\mu$ e $\sigma$. Porém, para obter um melhor ajuste é necessário procurar outro tipo de distribuição assimétrica similar à gama, mas provavelmente com mais parâmetros. Sendo assim, podemos utilizar a distribuição gama generalizada com três parâmetros, disponível no gamlss, para verificar se o modelo fica melhor ajustado.

A parametrização da distribuição gama generalizada utilizada no gamlss é denotada por $\mathrm{GG}(\mu, \sigma, \nu)$ e sua função densidade de probabilidade é definida por

$$
f(y \mid \mu, \sigma, \nu)=\frac{\theta^{\theta} z^{\theta}|\nu| e^{-\theta z}}{\Gamma(\theta) y}
$$

em que $z=\left(\frac{y}{\mu}\right)^{\nu}$ e $\theta=\frac{1}{\sigma^{2} \nu^{2}}$, para $y>0, \mu>0, \sigma>0$ e $-\infty<\nu<\infty$, com $\nu \neq 0$, sendo $\mu$ o 
Tabela 4.6: Estimativas de máxima verossimilhança penalizadas referentes ao modelo utilizando a distribuição gama generalizada ajustado, seus respectivos erros padrão aproximados e valores-p, aos dados Valor do Aluguel.

\begin{tabular}{lrrr}
\hline Efeito & Estimativa & Erro padrão & Valor-p \\
\hline Intercepto & $-1,24$ & 0,34 & 0,00 \\
localBoa & 0,10 & 0,01 & 0,00 \\
localExcelente & 0,23 & 0,03 & 0,00 \\
banhPremium & 0,07 & 0,02 & 0,01 \\
cozPremium & 0,13 & 0,02 & 0,00 \\
Com Aquec. & 0,30 & 0,02 & 0,00 \\
\hline \hline P-spline(area) & 0,01 & & 0,00 \\
P-spline(ano) & 0,00 & & 0,00 \\
$d f(\boldsymbol{\lambda})$ & 20,23 & & \\
$d f_{\gamma}($ area $)$ & 6,61 & & \\
$d f_{\gamma}($ ano $)$ & 5,62 & & \\
\hline \hline
\end{tabular}

parâmetro de localização, $\sigma$ o parâmetro de escala e $\nu$ o parâmetro de forma.

Sendo assim, vamos propor inicialmente o seguinte modelo para a média:

$$
\begin{gathered}
y_{i} \mid \mathbf{x}_{i} \stackrel{\text { ind }}{\sim} G G\left(\mu_{i}, \sigma, \nu\right) \text { com } \\
\log \left(\mu_{i}\right)=\beta_{0}+\beta_{11} \operatorname{loc}_{1 i}+\beta_{12} \operatorname{loc}_{2 i}+\beta_{2} \text { banh }_{i}+\beta_{3} \text { coz }_{i}+\beta_{4} \text { aquec }_{i}+f_{1}\left(\text { area }_{i}\right)+f_{2}\left(\text { ano }_{i}\right),
\end{gathered}
$$

em que $\mathbf{x}_{i}=\left(1, \text { loc }_{1 i}, \text { loc }_{2 i}, \text { banh }_{i}, \text { coz }_{i}, \text { aquec }_{i}, \text { area }_{i}, \text { ano }_{i}\right)^{\top}$.

As variáveis de comportamento não paramétrico foram ajustadas por meio de $\mathrm{P}$-splines considerando B-splines cúbicos, a penalidade de diferenças de segunda ordem e o método GAIC para estimar os parâmetros de suavização $\boldsymbol{\lambda}$.

As estimativas de máxima verossimilhança penalizadas para os parâmetros do modelo (4.3), obtidas por meio do gamlss, são apresentadas na Tabela 4.6. Para as variáveis explicativas área e ano de construção, tratadas de forma não linear, as estimativas fornecidas na tabela referemse à parte linear da função de suavização e não à sua contribuição total, sendo esta obtida na Figura 4.11. Por definição, os graus de liberdade efetivos obtidos no ajuste são dados por $d f(\boldsymbol{\lambda})=$ $6+1+1+d f_{\gamma}($ area $)+d f_{\gamma}($ ano $)$, em que 6 se refere ao intercepto e às variáveis explicativas tratadas de forma linear e $1+1$ se refere aos parâmetros $\sigma$ e $\nu$.

Para os fatores, nota-se pela Tabela 4.6 que quanto melhor a localização do imóvel, maior é o valor do aluguel, assim como a presença de aquecimento central e melhores instalações de banheiro e cozinha fazem com que o valor do aluguel seja maior. Além disso, vemos pela Figura 4.11, que quanto maior a área do apartamento, maior o aluguel cobrado. Para a variável ano de construção, nota-se que o valor médio do aluguel para apartamentos construídos a partir de 1960 fica mais caro à medida que a construção é mais recente, sendo que antes desse ano, o valor do aluguel varia pouco.

Para avaliar a qualidade do ajuste, a análise de diagnóstico é apresentada na Figura 4.12. Pelo gráfico normal de probabilidades (Q-Q plot) vemos que os resíduos possuem um comportamento aproximadamente linear, de forma que a assimetria à esquerda observada nos ajustes anteriores foi mais controlada ao utilizar a distribuição gama generalizada com três parâmetros, porém ainda existe um pouco de cauda pesada. Pelo gráfico de resíduos quantílicos contra o valor ajustado vemos 

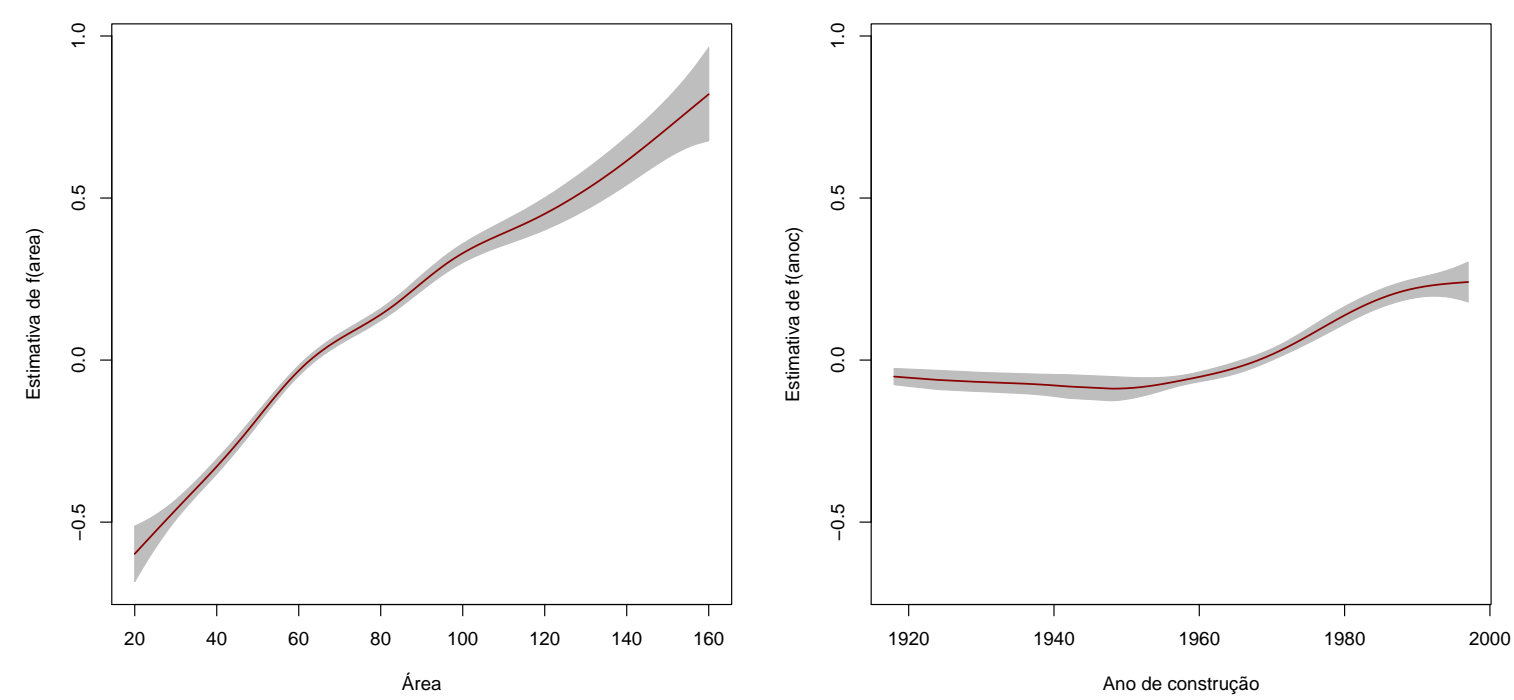

Figura 4.11: Bandas de confiança pontuais aproximadas de $95 \%$ para os efeitos das variáveis explicativas área e ano de construção no valor médio do aluguel referentes ao modelo utilizando a distribuição gama gemeralizada ajustado aos dados Valor do Aluguel.

que, do mesmo modo que ocorreu no ajuste do modelo (1.5), a variabilidade não foi totalmente controlada porque ajustamos apenas a média $\mu$, mantendo o coeficiente de variação $\sigma$ constante. A fim de melhorar o ajuste obtido, vamos modelar a média $\mu$ conjuntamente com o $\sigma$ (modelo duplo), mantendo o parâmetro $\nu$ constante.

Sendo assim, vamos propor o seguinte modelo para $\mu$ e $\sigma$ :

$$
y_{i} \mid \mathbf{x}_{i} \stackrel{i n d}{\sim} G G\left(\mu_{i}, \sigma_{i}, \nu\right)
$$

em que

$$
\begin{aligned}
& \log \left(\mu_{i}\right)=\beta_{10}+\beta_{11} \text { loc }_{1 i}+\beta_{12} \text { loc }_{2 i}+\beta_{13} \text { banh }_{i}+\beta_{14} \text { coz }_{i}+\beta_{15} \text { aquec }_{i}+f_{1}^{\mu}\left(\text { area }_{i}\right)+f_{2}^{\mu}\left(\text { ano }_{i}\right) \text { e }
\end{aligned}
$$

Utilizando o critério de seleção de modelos GAIC temos que o modelo final é dado por

$$
\begin{gathered}
\log \left(\mu_{i}\right)=\beta_{10}+\beta_{11} \text { loc }_{1 i}+\beta_{12} \text { loc }_{2 i}+\beta_{13} \text { banh }_{i}+\beta_{14} \text { coz }_{i}+\beta_{15} \text { aquec }_{i}+f_{1}^{\mu}\left(\text { area }_{i}\right)+f_{2}^{\mu}\left(\text { ano }_{i}\right) \mathrm{e} \\
\log \left(\sigma_{i}\right)=\beta_{20}+\beta_{21} \text { loc }_{1 i}+\beta_{22} \text { loc }_{2 i}+\beta_{23} \text { aquec }_{i}+f_{1}^{\sigma}\left(\text { area }_{i}\right)+f_{2}^{\sigma}\left(\text { ano }_{i}\right),
\end{gathered}
$$

sendo $\mathbf{x}_{i}=\left(1, \text { loc }_{1 i}, \text { loc }_{2 i}, \text { banh }_{i}, \text { coz }_{i}, \text { aquec }_{i}, \text { area }_{i}, \text { ano }_{i}\right)^{\top}$.

Novamente vemos que para modelar o coeficiente de variação $\sigma$ apenas as variáveis área, ano de construção, localização e aquecimento central são significativas. Para a localização $\mu$, todas as variáveis são significativas.

As estimativas de máxima verossimilhança penalizadas para os parâmetros do modelo (4.5) são apresentadas nas Tabelas 4.7 e 4.8. Além disso, a contribuição total das variáveis explicativas 



Figura 4.12: Gráficos de resíduos referentes ao modelo utilizando a distribuição gama generalizada ajustado aos dados Valor do Aluguel. 
Tabela 4.7: Estimativas de máxima verossimilhança penalizadas para os efeitos da localização referentes ao modelo duplo utilizando a distribuição gama generalizada ajustado, seus respectivos erros padrão aproximados e valores-p, aos dados Valor do Aluguel.

\begin{tabular}{lrrr}
\hline Efeito & Estimativa & Erro padrão & Valor-p \\
\hline Intercepto & $-1,61$ & 0,03 & 0,00 \\
localBoa & 0,09 & 0,01 & 0,00 \\
localExcelente & 0,22 & 0,04 & 0,00 \\
banhPremium & 0,06 & 0,02 & 0,00 \\
cozPremium & 0,10 & 0,02 & 0,00 \\
Com Aquec. & 0,29 & 0,02 & 0,00 \\
\hline \hline P-spline(area) & 0,01 & & 0,00 \\
P-spline(ano) & 0,00 & & 0,00 \\
$d f(\boldsymbol{\lambda})$ & 26,09 & & \\
$d f\left(\boldsymbol{\lambda}_{\mu}\right)$ & 19,08 & & \\
$d f_{\gamma}\left(\right.$ area $\left._{\mu}\right)$ & 6,63 & & \\
$d f_{\gamma}\left(\right.$ ano $\left._{\mu}\right)$ & 6,45 & & \\
\hline \hline
\end{tabular}

tratadas de forma não paramétrica é fornecida nas Figuras 4.13 e 4.14. Com relação aos graus de liberdade efetivos obtidos no ajuste, temos que $d f(\boldsymbol{\lambda})=d f\left(\boldsymbol{\lambda}_{\mu}\right)+d f\left(\boldsymbol{\lambda}_{\sigma}\right)+1=\left[6+d f_{\gamma}\left(\right.\right.$ area $\left._{\mu}\right)+$ $\left.d f_{\gamma}\left(\operatorname{ano}_{\mu}\right)\right]+\left[4+d f_{\gamma}\left(\right.\right.$ area $\left._{\sigma}\right)+d f_{\gamma}\left(\right.$ ano $\left.\left._{\sigma}\right)\right]+1=26,09$, em que 1 se refere ao coeficiente de forma $\nu$. Assim como ocorreu no modelo (4.1), os graus de liberdade das variáveis área do apartamento e ano de construção referentes ao ajuste do coeficiente de variação são bem próximos de 1 , o que nos dá indícios de que o comportamento do coeficiente de variação com relação à essas duas variáveis é linear.

Para o modelo proposto para $\mu$, nota-se pela Figura 4.13 que quanto maior a área do apartamento, maior o valor do aluguel cobrado. Além disso, para apartamentos construídos a partir de 1960 o valor do aluguel é maior para construções mais recente. Já para os fatores, vemos na Tabela 4.7 que apartamentos com uma localização melhor, com melhores instalações de banheiro e cozinha e que possuem aquecimento central são em média mais caros.

Na Figura 4.14 são apresentados os ajustes das funções suavizadas para o modelo proposto para $\sigma$. Nota-se que quanto maior a área do apartamento, maior é o coeficiente de variação do valor do aluguel. Em contrapartida, para a variável ano de construção, vemos que quanto mais recente é a construção do apartamento, menor é o coeficiente de variação do valor do aluguel. Pelos gráficos, é possível notar que a relação entre o coeficiente de variação e essas duas variáveis explicativas tem comportamento linear, sendo assim, para modelar o coeficiente de variação, poderíamos tratar as variáveis área e ano de construção de forma paramétrica. Com relação às estimativas dos fatores, fornecidas na Tabela 4.8, vemos que o coeficiente de variação do valor do aluguel aumenta quando a localização do apartamento é melhor e diminui quando o apartamento possui aquecimento central.

\subsubsection{Diagnóstico}

Para verificar a qualidade do ajuste do modelo (4.5), vamos realizar uma análise de diagnóstico. A Figura 4.15 apresenta os gráficos de resíduos fornecidos pelo gamlss.

Nota-se pelo gráfico de resíduos contra o valor ajustado que os resíduos se distribuem aleato- 
Tabela 4.8: Estimativas de máxima verossimilhança penalizadas para os efeitos do coeficiente de variação referentes ao modelo duplo utilizando a distribuição gama generalizada ajustado, seus respectivos erros padrão aproximados e valores-p, aos dados Valor do Aluguel.

\begin{tabular}{lrrr}
\hline Efeito & Estimativa & Erro padrão & Valor-p \\
\hline Intercepto & 9,19 & 0,08 & 0,00 \\
localBoa & 0,04 & 0,03 & 0,08 \\
localExcelente & 0,19 & 0,08 & 0,01 \\
Com Aquec. & $-0,23$ & 0,04 & 0,00 \\
\hline \hline P-spline(area) & 0,00 & & 0,02 \\
P-spline(ano) & $-0,01$ & & 0,00 \\
$d f(\boldsymbol{\lambda})$ & 26,09 & & \\
$d f\left(\boldsymbol{\lambda}_{\sigma}\right)$ & 6,02 & & \\
$d f_{\gamma}\left(\operatorname{area}_{\sigma}\right)$ & 1,01 & & \\
$d f_{\gamma}\left(\right.$ nno $\left._{\sigma}\right)$ & 1,01 & & \\
\hline \hline
\end{tabular}
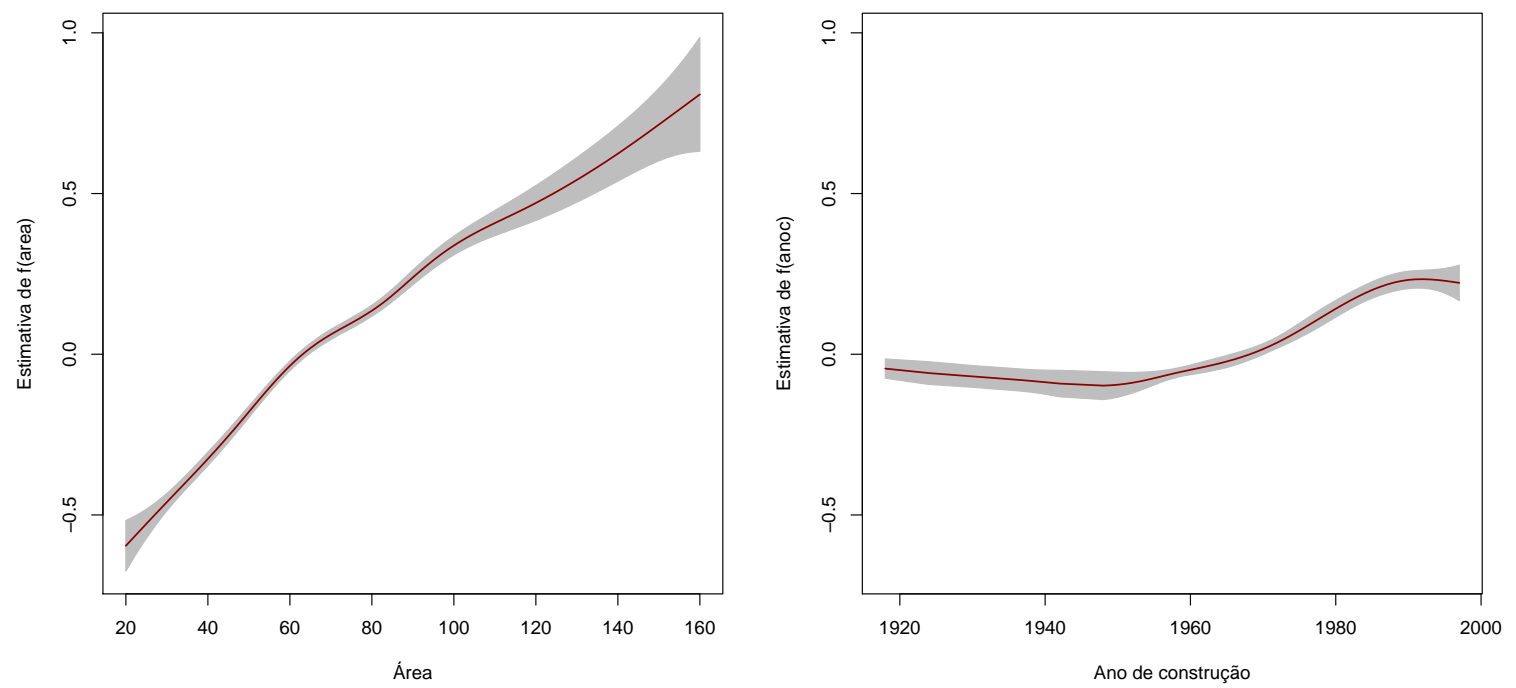

Figura 4.13: Bandas de confiança pontuais aproximadas de $95 \%$ para os efeitos das variáveis explicativas área e ano de construção no valor médio do aluguel referentes ao modelo duplo utilizando a distribuição gama generalizada ajustado aos dados Valor do Aluguel. 

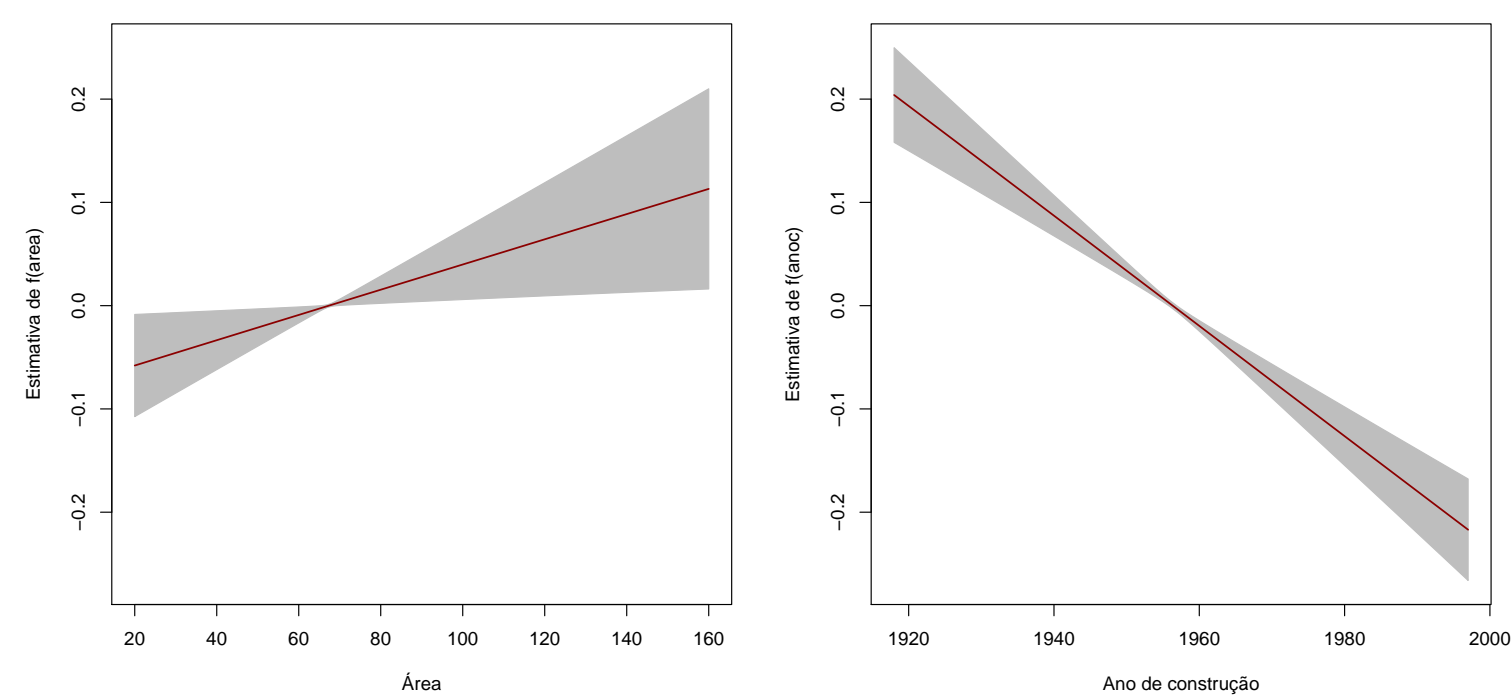

Figura 4.14: Bandas de confiança pontuais aproximadas de $95 \%$ para os efeitos das variáveis explicativas área e ano de construção no coeficiente de variação do aluguel referentes ao modelo duplo utilizando a distribuição gama generalizada ajustado aos dados Valor do Aluguel.

riamente em torno de zero, indicando que ao ajustar conjuntamente $\mu$ e $\sigma$ houve uma melhora no controle da variabilidade. No gráfico da estimativa da densidade vemos que os resíduos apresentam um comportamento aproximadamente normal. Além disso, pelo gráfico Q-Q plot nota-se que, apesar de alguns pontos estarem fora da diagonal, os resíduos apresentam um comportamento aproximadamente linear, porém ainda existe um pouco de cauda pesada, indicando que a assimetria à esquerda não foi totalmente controlada quando utilizou-se a distribuição gama generalizada de três parâmetros.

Sendo assim, comparando os modelos propostos para esses dados, vemos que os resultados inferenciais não se modificaram, mas que houve ganho ao utilizar a distribuição gama generalizada de três parâmetros e ao modelar conjuntamente $\mu$ e $\sigma$. Mesmo assim, para obter um melhor ajuste poderíamos utilizar outro tipo de distribuição, como por exemplo, a log-simétrica-t, discutida por Vanegas e Paula (2016), ou ainda aplicar o critério $\operatorname{GAIC}(\boldsymbol{\lambda})$ até obter um ajuste mais adequado. 



Figura 4.15: Gráficos de resíduos referentes ao modelo duplo utilizando a distribuição gama generalizada ajustado aos dados Valor do Aluguel. 


\section{Capítulo 5}

\section{Conclusões}

\subsection{Considerações finais}

Neste trabalho apresentamos os modelos lineares parciais generalizados e os modelos lineares parciais aditivos generalizados utilizando o método de suavização P-spline para o ajuste da parte não paramétrica dos modelos. O objetivo principal é detalhar a teoria desses modelos na literatura e aplicar um método de suavização diferente dos splines cúbicos com penalização de segunda derivada, conforme descrito em Green e Silverman (1994), que são bastante utilizados.

Nos Capítulos 2 e 3 estão as maiores contribuições desta dissertação. No Capítulo 2 apresentamos os modelos lineares parciais generalizados, a teoria do método de suavização P-splines e o seu critério de penalização, que consiste no cálculo das diferenças entre os coeficientes adjacentes das bases de De Boor. Além disso, apresentamos uma outra forma de penalização muito utilizada na literatura que envolve os splines cúbicos e discutimos alguns benefícios da utilização dos P-splines ao invés dos splines cúbicos. Uma vez discutido o método de suavização, definimos a função de verossimilhança penalizada e obtemos as funções escore e as matrizes de informação de Fisher penalizadas. A estimação dos parâmetros do modelo foi realizada por meio da combinação entre o processo iterativo escore de Fisher e o algoritmo backfitting. Além disso, discutimos a estimação dos graus de liberdade efetivos do ajuste e algumas maneiras de estimar o parâmetro de suavização do modelo. Por fim, os resultados assintóticos, análise de resíduos e testes de hipóteses, em particular para verificar se a parte não paramétrica do modelo é significativa, são apresentados.

Já no Capítulo 3, apresentamos os modelos lineares parciais aditivos generalizados que são uma extensão dos modelos lineares parciais generalizados. Nesse capítulo, obtemos as funções escore e matriz de informação de Fisher penalizadas para o caso em que há mais de uma variável explicativa no modelo a ser tratada de forma não paramétrica. As estimativas de máxima verossimilhança penalizadas dos parâmetros também foram obtidas por meio da combinação do processo iterativo escore de Fisher e o algoritmo backfitting. Em seguida, discutimos novamente como obter os graus de liberdade efetivos do modelo e algumas maneiras para estimar os parâmetros de suavização de cada função do modelo. Por fim, apresentamos testes de hipóteses para essa classe de modelos.

No ponto de vista prático, no Capítulo 4 aplicamos a teoria discutida durante essa dissertação aos três bancos de dados introduzidos no Capítulo 1, que possuíam variáveis com comportamento não linear, por meio do pacote gamlss. Verificamos por meio das análises de diagnóstico que os modelos lineares parciais generalizados e parciais aditivos generalizados utilizando P-splines se ajustaram bem aos dados, porém em um dos casos é sugerido que o ajuste seja feito utilizando outra 
distribuição, como por exemplo, a log-simétrica-t, ou ainda que se utilize o critério de informação generalizado para se obter um melhor ajuste.

\subsection{Sugestões para pesquisas futuras}

Uma extensão natural deste trabalho seria aplicar metodologias de sensibilidade, por exemplo, deteç̧ão de pontos aberrantes e influência local, nos MLPGs e MLPAGs e implementar o procedimento no software $\mathrm{R}$ ou mesmo na biblioteca gamlss. Do ponto de vista empírico seria de interesse estudar as propriedades dos estimadores de máxima verossimilhança penalizada para amostras pequenas e moderadas nessas duas classes de modelos. Todos esses estudos poderiam ser estendidos a uma variedade de distribuições univariadas disponíveis na biblioteca gamlss.

Somada à essas sugestões, pode-se pesquisar a aplicação do método P-splines utilizando outras penalizações, como por exemplo, as circulares. Além disso, poderia ser estudada a utilização dos P-splines por meio da abordagem Bayesiana. Por fim, outra sugestão seria pesquisar o ajuste do componente não paramétrico utilizando outros métodos de suavização diferente dos P-splines, como por exemplo, os O-splines (Wand e Ormerod, 2008). 


\section{Apêndice A}

\section{Derivações do MLPG}

Neste apêndice apresentamos os detalhes dos cálculos necessários para obter as funções escore penalizadas e a informação de Fisher penalizada para os modelos lineares parciais generalizados, definidas na Seção 2.4.2.

Na Seção 2.4.1, vimos que o logaritmo da função de verossimilhança penalizada dos MLPGs usando P-splines é definido por

$$
L_{p}(\boldsymbol{\theta}, \lambda)=\sum_{i=1}^{n}\left[\phi\left\{y_{i} \theta_{i}-b\left(\theta_{i}\right)\right\}+c\left(y_{i} ; \phi\right)\right]-\frac{\lambda}{2} \boldsymbol{\gamma}^{\top} \mathbf{P}_{d} \boldsymbol{\gamma}
$$

em que $\boldsymbol{\theta}=\left(\boldsymbol{\beta}^{\top}, \boldsymbol{\gamma}^{\top}, \phi\right)^{\top}$ é o vetor de parâmetros a serem estimados.

\section{A.1 Funções escore penalizadas}

Nesta seção vamos apresentar os cálculos para a obtenção das funções escore penalizadas.

A função escore penalizada $\mathbf{U}_{p}^{\boldsymbol{\theta}}(\boldsymbol{\theta})$ é definida por

$$
\mathbf{U}_{p}^{\boldsymbol{\theta}}(\boldsymbol{\theta})=\left(\begin{array}{c}
\mathbf{U}_{p}^{\boldsymbol{\beta}}(\boldsymbol{\theta}) \\
\mathbf{U}_{p}^{\gamma}(\boldsymbol{\theta}) \\
\mathrm{U}_{p}^{\phi}(\boldsymbol{\theta})
\end{array}\right)
$$

Começando pelo cálculo da função escore penalizada referente à parte paramétrica $\mathbf{U}_{p}^{\boldsymbol{\beta}}(\boldsymbol{\theta})$, vamos derivar (A.1) em relação ao vetor $\boldsymbol{\beta}$. Temos que

$$
\begin{aligned}
\mathrm{U}_{p}^{\beta_{j}}(\boldsymbol{\theta})=\frac{\partial L_{p}(\boldsymbol{\theta}, \lambda)}{\partial \beta_{j}} & =\sum_{i=1}^{n}\left[\phi\left\{y_{i} \frac{d \theta_{i}}{d \mu_{i}} \frac{d \mu_{i}}{d \eta_{i}} \frac{\partial \eta_{i}}{\partial \beta_{j}}-\frac{d b\left(\theta_{i}\right)}{d \theta_{i}} \frac{d\left(\theta_{i}\right)}{d \mu_{i}} \frac{d \mu_{i}}{d \eta_{i}} \frac{\partial \eta_{i}}{\partial \beta_{j}}\right\}\right] \\
& =\sum_{i=1}^{n} \phi\left(y_{i} \frac{d \mu_{i} / d \eta_{i}}{V_{i}} x_{i j}-\mu_{i} \frac{d \mu_{i} / d \eta_{i}}{V_{i}} x_{i j}\right) \\
& =\phi \sum_{i=1}^{n} \sqrt{\frac{w_{i}}{V_{i}}}\left(y_{i}-\mu_{i}\right) x_{i j}, \text { para } j=1, \ldots, p .
\end{aligned}
$$

Escrevendo a equação acima em forma matricial, obtemos

$$
\mathbf{U}_{p}^{\boldsymbol{\beta}}(\boldsymbol{\theta})=\frac{\partial L_{p}(\boldsymbol{\theta}, \lambda)}{\partial \boldsymbol{\beta}}=\phi \mathbf{X}^{\top} \mathbf{W}^{\frac{1}{2}} \mathbf{V}^{-\frac{1}{2}}(\mathbf{y}-\boldsymbol{\mu}) .
$$


Para o vetor $\boldsymbol{\gamma}$ de efeito não paramétrico, temos que a função escore penalizada $\mathbf{U}_{p}^{\gamma}(\boldsymbol{\theta})$ fica dada por

$$
\begin{aligned}
\mathrm{U}_{p}^{\gamma_{j}}(\boldsymbol{\theta})=\frac{\partial L_{p}(\boldsymbol{\theta}, \lambda)}{\partial \gamma_{j}} & =\sum_{i=1}^{n} \phi\left\{y_{i} \frac{d \theta_{i}}{d \mu_{i}} \frac{d \mu_{i}}{d \eta_{i}} \frac{\partial \eta_{i}}{\partial \gamma_{j}}-\frac{d b\left(\theta_{i}\right)}{d \theta_{i}} \frac{d \theta_{i}}{d \mu_{i}} \frac{d \mu_{i}}{d \eta_{i}} \frac{\partial \eta_{i}}{\partial \gamma_{j}}\right\}-\left[\lambda \mathbf{P}_{d} \boldsymbol{\gamma}\right]_{j} \\
& =\sum_{i=1}^{n} \phi\left\{y_{i} \frac{d \mu_{i} / d \eta_{i}}{V_{i}} b_{i j}-\mu_{i} \frac{d \mu_{i} / d \eta_{i}}{V_{i}} b_{i j}\right\}-\left[\lambda \mathbf{P}_{d} \gamma\right]_{j} \\
& =\phi \sum_{i=1}^{n} \sqrt{\frac{w_{i}}{V_{i}}}\left(y_{i}-\mu_{i}\right) b_{i j}-\left[\lambda \mathbf{P}_{d} \boldsymbol{\gamma}\right]_{j}, \text { para } j=1, \ldots, q .
\end{aligned}
$$

Em forma matricial, obtemos

$$
\mathbf{U}_{p}^{\gamma}(\boldsymbol{\theta})=\frac{\partial L_{p}(\boldsymbol{\theta}, \lambda)}{\partial \boldsymbol{\gamma}}=\phi \mathbf{B}^{\top} \mathbf{W}^{\frac{1}{2}} \mathbf{V}^{-\frac{1}{2}}(\mathbf{y}-\boldsymbol{\mu})-\lambda \mathbf{P}_{d} \boldsymbol{\gamma} .
$$

Por fim, para o parâmetro de precisão $\phi$, temos a seguinte função escore penalizada:

$$
\mathrm{U}_{p}^{\phi}(\boldsymbol{\theta})=\frac{\partial L_{p}(\boldsymbol{\theta}, \lambda)}{\partial \phi}=\sum_{i=1}^{n}\left\{y_{i} \theta_{i}-b\left(\theta_{i}\right)\right\}+\sum_{i=1}^{n} c^{\prime}\left(y_{i} ; \phi\right),
$$

em que $c^{\prime}\left(y_{i} ; \phi\right)=\frac{d c\left(y_{i} ; \phi\right)}{d \phi}$.

\section{A.2 Matriz de informação de Fisher penalizada}

Uma vez obtidas as derivadas de primeira ordem, vamos derivar nesta seção a matriz de informação de Fisher penalizada.

Definindo a matriz de informação de Fisher penalizada para $\boldsymbol{\theta}$, temos que

$$
\mathbf{K}_{p}^{\boldsymbol{\theta} \boldsymbol{\theta}}(\boldsymbol{\theta})=E\left\{-\frac{\partial^{2} L_{p}(\boldsymbol{\theta}, \lambda)}{\partial \boldsymbol{\theta} \partial \boldsymbol{\theta}^{\top}}\right\}
$$

em que

$$
\mathbf{K}_{p}^{\boldsymbol{\theta} \boldsymbol{\theta}}(\boldsymbol{\theta})=\left(\begin{array}{ccc}
\mathbf{K}_{p}^{\boldsymbol{\beta} \boldsymbol{\beta}}(\boldsymbol{\theta}) & \mathbf{K}_{p}^{\boldsymbol{\beta} \boldsymbol{\gamma}}(\boldsymbol{\theta}) & \mathbf{K}_{p}^{\boldsymbol{\beta} \phi}(\boldsymbol{\theta}) \\
\mathbf{K}_{p}^{\boldsymbol{\gamma} \boldsymbol{\beta}}(\boldsymbol{\theta}) & \mathbf{K}_{p}^{\gamma \boldsymbol{\gamma}}(\boldsymbol{\theta}) & \mathbf{K}_{p}^{\boldsymbol{\gamma} \phi}(\boldsymbol{\theta}) \\
\mathbf{K}_{p}^{\phi \boldsymbol{\beta}}(\boldsymbol{\theta}) & \mathbf{K}_{p}^{\phi \boldsymbol{\gamma}}(\boldsymbol{\theta}) & \mathbf{K}_{p}^{\phi \phi}(\boldsymbol{\theta})
\end{array}\right)
$$

Primeiramente, vamos derivar a matriz de informação de Fisher para o parâmetro $\boldsymbol{\beta}$. Temos que

$$
\mathrm{K}_{p}^{\beta_{j} \beta_{l}}(\boldsymbol{\theta})=E\left\{-\frac{\partial^{2} L_{p}(\boldsymbol{\theta}, \lambda)}{\partial \beta_{j} \partial \beta_{l}}\right\} .
$$

Derivando a expressão (A.2) com relação à $\boldsymbol{\beta}$, obtemos 


$$
\begin{aligned}
\frac{\partial^{2} L_{p}(\boldsymbol{\theta}, \lambda)}{\partial \beta_{j} \partial \beta_{l}} & =\frac{\partial}{\partial \beta_{l}}\left\{\phi \sum_{i=1}^{n} \frac{d \theta_{i}}{d \mu_{i}} \frac{d \mu_{i}}{d \eta_{i}}\left(y_{i}-\mu_{i}\right) x_{i j}\right\}= \\
& =\phi \sum_{i=1}^{n} \frac{d^{2} \theta_{i}}{d \mu_{i}^{2}}\left(\frac{d \mu_{i}}{d \eta_{i}}\right)^{2} \frac{\partial \eta_{i}}{\partial \beta_{l}}\left(y_{i}-\mu_{i}\right) x_{i j}+\phi \sum_{i=1}^{n} \frac{d \theta_{i}}{d \mu_{i}} \frac{d^{2} \mu_{i}}{d \eta_{i}^{2}} \frac{\partial \eta_{i}}{\partial \beta_{l}}\left(y_{i}-\mu_{i}\right) x_{i j}- \\
& -\sum_{i=1}^{n} \phi \frac{d \theta_{i}}{d \mu_{i}}\left(\frac{d \mu_{i}}{d \eta_{i}}\right)^{2} \frac{\partial \eta_{i}}{\partial \beta_{l}} x_{i j}= \\
& =\phi \sum_{i=1}^{n} \frac{d^{2} \theta_{i}}{d \mu_{i}^{2}}\left(\frac{d \mu_{i}}{d \eta_{i}}\right)^{2}\left(y_{i}-\mu_{i}\right) x_{i j} x_{i l}+\phi \sum_{i=1}^{n} \frac{d \theta_{i}}{d \mu_{i}} \frac{d^{2} \mu_{i}}{d \eta_{i}^{2}}\left(y_{i}-\mu_{i}\right) x_{i j} x_{i l}- \\
& -\sum_{i=1}^{n} \phi \frac{d \theta_{i}}{d \mu_{i}}\left(\frac{d \mu_{i}}{d \eta_{i}}\right)^{2} x_{i j} x_{i l} .
\end{aligned}
$$

Sendo assim,

$$
\begin{aligned}
\mathrm{K}_{p}^{\beta_{j} \beta_{l}}(\boldsymbol{\theta}) & =E\left\{-\frac{\partial^{2} L_{p}(\boldsymbol{\theta}, \lambda)}{\partial \beta_{j} \partial \beta_{l}}\right\}= \\
& =0+0+E\left\{\phi \sum_{i=1}^{n} \frac{d \theta_{i}}{d \mu_{i}}\left(\frac{d \mu_{i}}{d \eta_{i}}\right)^{2} x_{i j} x_{i l}\right\}= \\
& =\phi \sum_{i=1}^{n} w_{i} x_{i j} x_{i l}, \text { para } j, l=1, \ldots, p .
\end{aligned}
$$

Escrevendo em forma matricial, temos que a matriz de informação de Fisher $\mathbf{K}_{p}^{\boldsymbol{\beta} \boldsymbol{\beta}}(\boldsymbol{\theta})$ é definida por

$$
\mathbf{K}_{p}^{\boldsymbol{\beta} \boldsymbol{\beta}}(\boldsymbol{\theta})=\phi \mathbf{X}^{\top} \mathbf{W X}
$$

Para o parâmetro $\gamma$, temos que

$$
\mathrm{K}_{p}^{\gamma_{j} \gamma_{l}}(\boldsymbol{\theta})=E\left\{-\frac{\partial^{2} L_{p}(\boldsymbol{\theta}, \lambda)}{\partial \gamma_{j} \partial \gamma_{l}}\right\}
$$

Obtendo a segunda derivada da equação (A.3) em relação ao parâmetro $\gamma$ do componente não paramétrico, temos que

$$
\begin{aligned}
\frac{\partial^{2} L_{p}(\boldsymbol{\theta}, \lambda)}{\partial \gamma_{j} \partial \gamma_{l}} & =\frac{\partial}{\partial \gamma_{l}}\left\{\phi \sum_{i=1}^{n} \frac{d \theta_{i}}{d \mu_{i}} \frac{d \mu_{i}}{d \eta_{i}}\left(y_{i}-\mu_{i}\right) b_{i j}-\left[\lambda \mathbf{P}_{d} \gamma\right]_{j}\right\}= \\
& =\phi \sum_{i=1}^{n} \frac{d^{2} \theta_{i}}{d \mu_{i}^{2}}\left(\frac{d \mu_{i}}{d \eta_{i}}\right)^{2} \frac{\partial \eta_{i}}{\partial \gamma_{l}}\left(y_{i}-\mu_{i}\right) b_{i j}+\phi \sum_{i=1}^{n} \frac{d \theta_{i}}{d \mu_{i}} \frac{d^{2} \mu_{i}}{d \eta_{i}^{2}} \frac{\partial \eta_{i}}{\partial \gamma_{l}}\left(y_{i}-\mu_{i}\right) b_{i j}- \\
& -\phi \sum_{i=1}^{n}\left\{\frac{d \theta_{i}}{d \mu_{i}}\left(\frac{d \mu_{i}}{d \eta_{i}}\right)^{2} \frac{\partial \eta_{i}}{\partial \gamma_{l}} b_{i j}-\lambda p_{i j}\right\}= \\
& =\phi \sum_{i=1}^{n} \frac{d^{2} \theta_{i}}{d \mu_{i}^{2}}\left(\frac{d \mu_{i}}{d \eta_{i}}\right)^{2}\left(y_{i}-\mu_{i}\right) b_{i j} b_{i l}+\phi \sum_{i=1}^{n} \frac{d \theta_{i}}{d \mu_{i}} \frac{d^{2} \mu_{i}}{d \eta_{i}^{2}}\left(y_{i}-\mu_{i}\right) b_{i j} b_{i l}- \\
& -\phi \sum_{i=1}^{n}\left\{\frac{d \theta_{i}}{d \mu_{i}}\left(\frac{d \mu_{i}}{d \eta_{i}}\right)^{2} b_{i j} b_{i l}-\lambda p_{i j}\right\}, \text { para } j, l=1, \ldots, q .
\end{aligned}
$$

Desta forma, 


$$
\begin{aligned}
\mathrm{K}_{p}^{\gamma_{j} \gamma_{l}}(\boldsymbol{\theta}) & =E\left\{-\frac{\partial^{2} L_{p}(\boldsymbol{\theta}, \lambda)}{\partial \gamma_{j} \partial \gamma_{l}}\right\}= \\
& =0+0+E\left\{\phi \sum_{i=1}^{n} \frac{d \theta_{i}}{d \mu_{i}}\left(\frac{d \mu_{i}}{d \eta_{i}}\right)^{2} b_{i j} b_{i l}+\lambda p_{i j}\right\}= \\
& =\phi \sum_{i=1}^{n} w_{i} b_{i j} b_{i l}+\lambda p_{i j}, \text { para } j, l=1, \ldots, q .
\end{aligned}
$$

Escrevendo em forma matricial, a matriz de informação de Fisher $\mathbf{K}_{p}^{\gamma \gamma}(\boldsymbol{\theta})$ fica dada por

$$
\mathbf{K}_{p}^{\gamma \gamma}(\boldsymbol{\theta})=\phi \mathbf{B}^{\top} \mathbf{W B}+\lambda \mathbf{P}_{d}
$$

Para o parâmetro de precisão $\phi$, a informação de Fisher penalizada é definida por

$$
\mathrm{K}_{p}^{\phi \phi}(\boldsymbol{\theta})=E\left\{-\frac{\partial^{2} L_{p}(\boldsymbol{\theta}, \lambda)}{\partial \phi \partial \phi}\right\}
$$

Vamos calcular primeiramente a segunda derivada da equação (A.4) em relação à $\phi$. Assim, obtemos

$$
\begin{aligned}
\frac{\partial^{2} L_{p}(\boldsymbol{\theta}, \lambda)}{\partial \phi \partial \phi} & =\frac{\partial}{\partial \phi}\left[\sum_{i=1}^{n}\left\{y_{i} \theta_{i}-b\left(\theta_{i}\right)\right\}+\sum_{i=1}^{n} c^{\prime}\left(y_{i} ; \phi\right)\right]= \\
& =\sum_{i=1}^{n} c^{\prime \prime}\left(y_{i} ; \phi\right) .
\end{aligned}
$$

Dessa forma, temos que a informação de Fisher penalizada $\mathrm{K}_{p}^{\phi \phi}(\boldsymbol{\theta})$ fica dada por

$$
\mathrm{K}_{p}^{\phi \phi}(\boldsymbol{\theta})=E\left\{-\frac{\partial^{2} L_{p}(\boldsymbol{\theta}, \lambda)}{\partial \phi \partial \phi}\right\}=-\sum_{i=1}^{n} E\left\{c^{\prime \prime}\left(y_{i} ; \phi\right)\right\},
$$

em que $c^{\prime \prime}\left(y_{i} ; \phi\right)=\frac{d^{2} c\left(y_{i} ; \phi\right)}{d \phi^{2}}$

Após calcularmos os blocos diagonais da matriz de informação de Fisher penalizada de $\boldsymbol{\theta}$, vamos obter a matriz de informação de Fisher penalizada com parâmetros cruzados.

Primeiramente, vamos obter a informação de Fisher penalizada $K_{p}^{\beta_{j} \gamma_{l}}(\boldsymbol{\theta})$ que é definida por

$$
\mathrm{K}_{p}^{\beta_{j} \gamma_{l}}(\boldsymbol{\theta})=E\left\{-\frac{\partial^{2} L_{p}(\boldsymbol{\theta}, \lambda)}{\partial \beta_{j} \partial \gamma_{l}}\right\}, \text { para } j=1, \ldots, p \text { e } l=1, \ldots, q .
$$

Obtemos 


$$
\begin{aligned}
-\frac{\partial^{2} L_{p}(\boldsymbol{\theta}, \lambda)}{\partial \beta_{j} \partial \gamma_{l}} & =-\frac{\partial}{\partial \gamma_{l}}\left[\phi\left\{\sum_{i=1}^{n} \frac{d \theta_{i}}{d \mu_{i}} \frac{d \mu_{i}}{d \eta_{i}}\left(y_{i}-\mu_{i}\right) x_{i j}\right\}\right]= \\
& =-\phi \sum_{i=1}^{n} \frac{d^{2} \theta_{i}}{d \mu_{i}^{2}}\left(\frac{d \mu_{i}}{d \eta_{i}}\right)^{2} \frac{\partial \eta_{i}}{\partial \gamma_{l}}\left(y_{i}-\mu_{i}\right) x_{i j}-\phi \sum_{i=1}^{n} \frac{d \theta_{i}}{d \mu_{i}} \frac{d^{2} \mu_{i}}{d \eta_{i}^{2}} \frac{\partial \eta_{i}}{\partial \gamma_{l}}\left(y_{i}-\mu_{i}\right) x_{i j}+ \\
& +\phi \sum_{i=1}^{n} \frac{d \theta_{i}}{d \mu_{i}}\left(\frac{d \mu_{i}}{d \eta_{i}}\right)^{2} \frac{\partial \eta_{i}}{\partial \gamma_{l}} x_{i j}= \\
& =-\phi \sum_{i=1}^{n} \frac{d^{2} \theta_{i}}{d \mu_{i}^{2}}\left(\frac{d \mu_{i}}{d \eta_{i}}\right)^{2}\left(y_{i}-\mu_{i}\right) x_{i j} b_{i l}-\phi \sum_{i=1}^{n} \frac{d \theta_{i}}{d \mu_{i}} \frac{d^{2} \mu_{i}}{d \eta_{i}^{2}}\left(y_{i}-\mu_{i}\right) x_{i j} b_{i l}+ \\
& +\phi \sum_{i=1}^{n} \frac{d \theta_{i}}{d \mu_{i}}\left(\frac{d \mu_{i}}{d \eta_{i}}\right)^{2} x_{i j} b_{i l}, \text { para } j=1, \ldots, p \text { e } l=1, \ldots, q .
\end{aligned}
$$

Assim, temos que

$$
\begin{aligned}
\mathrm{K}_{p}^{\beta_{j} \gamma_{l}}(\boldsymbol{\theta}) & =E\left\{-\frac{\partial^{2} L_{p}(\boldsymbol{\theta}, \lambda)}{\partial \beta_{j} \partial \gamma_{l}}\right\}= \\
& =E\left\{\phi \sum_{i=1}^{n} w_{i} x_{i j} b_{i l}\right\}= \\
& =\phi \sum_{i=1}^{n} w_{i} x_{i j} b_{i l}, \text { para } j=1, \ldots, p \text { e } l=1, \ldots, q
\end{aligned}
$$

Em forma matricial, escrevemos

$$
\mathbf{K}_{p}^{\boldsymbol{\beta} \boldsymbol{\gamma}}(\boldsymbol{\theta})=\phi \mathbf{X}^{\top} \mathbf{W B} .
$$

A informação de Fisher penalizada $K_{p}^{\beta_{j} \phi}(\boldsymbol{\theta})$ fica dada por

$$
\mathrm{K}_{p}^{\beta_{j} \phi}(\boldsymbol{\theta})=E\left\{-\frac{\partial^{2} L_{p}(\boldsymbol{\theta}, \lambda)}{\partial \beta_{j} \partial \phi}\right\}, \text { para } j=1, \ldots, p .
$$

Vamos obter inicialmente a segunda derivada de (A.2) com relação à $\phi$. Obtemos

$$
\begin{aligned}
\frac{\partial^{2} L_{p}(\boldsymbol{\theta}, \lambda)}{\partial \beta_{j} \partial \phi} & =\frac{\partial}{\partial \phi}\left[\phi\left\{\sum_{i=1}^{n} \frac{d \theta_{i}}{d \mu_{i}} \frac{d \mu_{i}}{d \eta_{i}}\left(y_{i}-\mu_{i}\right) x_{i j}\right\}\right]= \\
& =\sum_{i=1}^{n} \frac{d \theta_{i}}{d \mu_{i}} \frac{d \mu_{i}}{d \eta_{i}}\left(y_{i}-\mu_{i}\right) x_{i j}= \\
& =\sum_{i=1}^{n} \sqrt{\frac{w_{i}}{V_{i}}}\left(y_{i}-\mu_{i}\right) x_{i j}, \text { para } j=1, \ldots, p .
\end{aligned}
$$

Com isso, temos o seguinte resultado:

$$
\begin{aligned}
\mathrm{K}_{p}^{\beta_{j} \phi}(\boldsymbol{\theta}) & =E\left\{-\frac{\partial^{2} L_{p}(\boldsymbol{\theta}, \lambda)}{\partial \beta_{j} \partial \phi}\right\}= \\
& =E\left\{-\sum_{i=1}^{n} \sqrt{\frac{w_{i}}{V_{i}}}\left(y_{i}-\mu_{i}\right) x_{i j}\right\}=0, \text { para } j=1, \ldots, p
\end{aligned}
$$


em que $c^{\prime \prime}\left(y_{i} ; \phi\right)=\frac{d^{2} c\left(y_{i} ; \phi\right)}{d \phi^{2}}$.

Por fim, vamos calcular $\mathrm{K}_{p}^{\gamma_{j} \phi}$ que é definida por

$$
\mathrm{K}_{p}^{\gamma_{j} \phi}(\boldsymbol{\theta})=E\left\{-\frac{\partial^{2} L_{p}(\boldsymbol{\theta}, \lambda)}{\partial \gamma_{j} \partial \phi}\right\} \text {, para } j=1, \ldots, q .
$$

A informação observada penalizada fica dada por

$$
\begin{aligned}
-\frac{\partial L_{p}(\boldsymbol{\theta}, \lambda)}{\partial \gamma_{j} \partial \phi} & =-\frac{\partial}{\partial \phi}\left[\phi\left\{\sum_{i=1}^{n} \frac{d \theta_{i}}{d \mu_{i}} \frac{d \mu_{i}}{d \eta_{i}}\left(y_{i}-\mu_{i}\right) b_{i j}\right\}-\left[\lambda \mathbf{P}_{d} \boldsymbol{\gamma}\right]_{j}\right]= \\
& =-\sum_{i=1}^{n} \frac{d \theta_{i}}{d \mu_{i}} \frac{d \mu_{i}}{d \eta_{i}}\left(y_{i}-\mu_{i}\right) b_{i j}=-\sum_{i=1}^{n} \sqrt{\frac{w_{i}}{V_{i}}}\left(y_{i}-\mu_{i}\right) b_{i j}, \text { para } j=1, \ldots, q
\end{aligned}
$$

Assim,

$$
\begin{aligned}
\mathrm{K}_{p}^{\gamma_{j} \phi}(\boldsymbol{\theta}) & =E\left\{-\frac{\partial L_{p}(\boldsymbol{\theta}, \lambda)}{\partial \gamma_{j} \partial \phi}\right\}= \\
& =E\left\{-\sum_{i=1}^{n} \sqrt{\frac{w_{i}}{V_{i}}}\left(y_{i}-\mu_{i}\right) b_{i j}\right\}=0, \text { para } j=1, \ldots, q
\end{aligned}
$$




\section{Apêndice B}

\section{Derivações do MLPAG}

Assim como fizemos no Apêndice A, vamos apresentar o detalhamento dos cálculos para obter as funções escore penalizadas e a matriz de informação de Fisher penalizada para os modelos lineares parciais aditivos generalizados, definidos na Seção 3.2.2.

Como visto anteriormente na Seção 3.2.1, temos que o logaritmo da função de verossimilhança penalizada para os MLPAGs é dado por

$$
L_{p}(\boldsymbol{\theta}, \boldsymbol{\lambda})=\sum_{i=1}^{n}\left[\phi\left\{y_{i} \theta_{i}-b\left(\theta_{i}\right)\right\}+c\left(y_{i} ; \phi\right)\right]-\sum_{l=1}^{r} \frac{\lambda_{l}}{2} \boldsymbol{\gamma}_{l}^{\top} \mathbf{P}_{l}^{d} \boldsymbol{\gamma}_{l}
$$

em que $\boldsymbol{\lambda}=\left(\lambda_{1}, \ldots, \lambda_{r}\right)^{\top}$.

\section{B.1 Funções escore penalizadas}

Nesta seção, vamos apresentar os cálculos para obtenção das funções escores penalizadas.

A função escore penalizada para $\boldsymbol{\theta}$ é definida por

$$
\mathbf{U}_{p}^{\boldsymbol{\theta}}(\boldsymbol{\theta})=\left(\begin{array}{c}
\mathbf{U}_{p}^{\boldsymbol{\beta}}(\boldsymbol{\theta}) \\
\mathbf{U}_{p}^{\gamma_{1}}(\boldsymbol{\theta}) \\
\vdots \\
\mathbf{U}_{p}^{\gamma_{r}}(\boldsymbol{\theta}) \\
\mathrm{U}_{p}^{\phi}(\boldsymbol{\theta})
\end{array}\right) .
$$

Inicialmente, vamos calcular a função escore do parâmetro $\boldsymbol{\beta}, \mathbf{U}_{p}^{\boldsymbol{\beta}}(\boldsymbol{\theta})$

$$
\begin{aligned}
\mathrm{U}_{p}^{\beta_{j}}(\boldsymbol{\theta})=\frac{\partial L_{p}(\boldsymbol{\theta}, \boldsymbol{\lambda})}{\partial \beta_{j}} & =\sum_{i=1}^{n}\left[\phi\left\{y_{i} \frac{d \theta_{i}}{d \mu_{i}} \frac{d \mu_{i}}{d \eta_{i}} \frac{\partial \eta_{i}}{\partial \beta_{j}}-\frac{d b\left(\theta_{i}\right)}{d \theta_{i}} \frac{d\left(\theta_{i}\right)}{d \mu_{i}} \frac{d \mu_{i}}{d \eta_{i}} \frac{\partial \eta_{i}}{\partial \beta_{j}}\right\}\right] \\
& =\sum_{i=1}^{n} \phi\left\{y_{i} \frac{d \mu_{i} / d \eta_{i}}{V_{i}} x_{i j}-\mu_{i} \frac{d \mu_{i} / d \eta_{i}}{V_{i}} x_{i j}\right\} \\
& =\phi \sum_{i=1}^{n} \sqrt{\frac{w_{i}}{V_{i}}}\left(y_{i}-\mu_{i}\right) x_{i j}, \text { para } j=1, \ldots, p .
\end{aligned}
$$

Escrevendo o resultado acima em forma matricial, obtemos

$$
\mathbf{U}_{p}^{\boldsymbol{\beta}}(\boldsymbol{\theta})=\frac{\partial L_{p}(\boldsymbol{\theta}, \boldsymbol{\lambda})}{\partial \boldsymbol{\beta}}=\phi \mathbf{X}^{\top} \mathbf{W}^{\frac{1}{2}} \mathbf{V}^{-\frac{1}{2}}(\mathbf{y}-\boldsymbol{\mu}) .
$$


Para o parâmetro $\gamma_{j l}$, obtemos

$$
\begin{aligned}
\mathrm{U}_{p}^{\gamma_{j l}}(\boldsymbol{\theta})=\frac{\partial L_{p}(\boldsymbol{\theta}, \boldsymbol{\lambda})}{\partial \gamma_{j l}} & =\sum_{i=1}^{n} \phi\left\{y_{i} \frac{d \theta_{i}}{d \mu_{i}} \frac{d \mu_{i}}{d \eta_{i}} \frac{\partial \eta_{i}}{\partial \gamma_{j l}}-\frac{d b\left(\theta_{i}\right)}{d \theta_{i}} \frac{d \theta_{i}}{d \mu_{i}} \frac{d \mu_{i}}{d \eta_{i}} \frac{\partial \eta_{i}}{\partial \gamma_{j l}}\right\}-\left[\lambda_{l} \mathbf{P}_{l}^{d} \boldsymbol{\gamma}_{l}\right]_{j} \\
& =\sum_{i=1}^{n} \phi\left\{y_{i} \frac{d \mu_{i} / d \eta_{i}}{V_{i}} b_{i j l}-\mu_{i} \frac{d \mu_{i} / d \eta_{i}}{V_{i}} b_{i j l}\right\}-\left[\lambda_{l} \mathbf{P}_{l}^{d} \boldsymbol{\gamma}_{l}\right]_{j} \\
& =\phi \sum_{i=1}^{n} \sqrt{\frac{w_{i}}{V_{i}}}\left(y_{i}-\mu_{i}\right) b_{i j l}-\left[\lambda_{l} \mathbf{P}_{l}^{d} \boldsymbol{\gamma}_{l}\right]_{j}, \text { para } j=1, \ldots, q_{l} \text { e } l=1, \ldots, r
\end{aligned}
$$

Em forma matricial, obtemos

$$
\mathbf{U}_{p}^{\gamma_{l}}(\boldsymbol{\theta})=\frac{\partial L_{p}(\boldsymbol{\theta}, \boldsymbol{\lambda})}{\partial \boldsymbol{\gamma}_{l}}=\phi \mathbf{B}_{l}^{\top} \mathbf{W}^{\frac{1}{2}} \mathbf{V}^{-\frac{1}{2}}(\mathbf{y}-\boldsymbol{\mu})-\lambda_{l} \mathbf{P}_{l}^{d} \boldsymbol{\gamma}_{l}
$$

$\operatorname{com} l=1, \ldots, r$.

Finalmente, para o parâmetro de precisão $\phi$, temos a seguinte função escore penalizada:

$$
\mathrm{U}_{p}^{\phi}(\boldsymbol{\theta})=\frac{\partial L_{p}(\boldsymbol{\theta}, \boldsymbol{\lambda})}{\partial \phi}=\sum_{i=1}^{n}\left\{y_{i} \theta_{i}-b\left(\theta_{i}\right)\right\}+\sum_{i=1}^{n} c^{\prime}\left(y_{i} ; \phi\right),
$$

sendo $c^{\prime}\left(y_{i} ; \phi\right)=\frac{d c\left(y_{i} ; \phi\right)}{d \phi}$.

\section{B.2 Matriz de informação de Fisher penalizada}

Nessa seção, apresentamos os cálculos detalhados para obtenção da matriz de informação de Fisher penalizada definida na Seção 3.2.2.

Definimos a matriz de informação de Fisher penalizada como

$$
\mathbf{K}_{p}^{\boldsymbol{\theta} \boldsymbol{\theta}}(\boldsymbol{\theta})=E\left\{-\frac{\partial^{2} L_{p}(\boldsymbol{\theta}, \boldsymbol{\lambda})}{\partial \boldsymbol{\theta} \partial \boldsymbol{\theta}^{\top}}\right\},
$$

em que

$$
\mathbf{K}_{p}^{\boldsymbol{\theta} \boldsymbol{\theta}}(\boldsymbol{\theta})=\left(\begin{array}{ccccc}
\mathbf{K}_{p}^{\boldsymbol{\beta} \boldsymbol{\beta}}(\boldsymbol{\theta}) & \mathbf{K}_{p}^{\boldsymbol{\beta} \boldsymbol{\gamma}_{1}}(\boldsymbol{\theta}) & \ldots & \mathbf{K}_{p}^{\boldsymbol{\beta} \boldsymbol{\gamma}_{r}}(\boldsymbol{\theta}) & \mathbf{K}_{p}^{\boldsymbol{\beta} \phi}(\boldsymbol{\theta}) \\
\mathbf{K}_{p}^{\boldsymbol{\gamma}_{1} \boldsymbol{\beta}}(\boldsymbol{\theta}) & \mathbf{K}_{p}^{\gamma_{1} \boldsymbol{\gamma}_{1}}(\boldsymbol{\theta}) & \ldots & \mathbf{K}_{p}^{\boldsymbol{\gamma}_{1} \boldsymbol{\gamma}_{\boldsymbol{r}}}(\boldsymbol{\theta}) & \mathbf{K}_{p}^{\boldsymbol{\gamma}_{1} \phi}(\boldsymbol{\theta}) \\
\vdots & \vdots & \ddots & \vdots & \vdots \\
\mathbf{K}_{p}^{\boldsymbol{\gamma}_{r} \boldsymbol{\beta}}(\boldsymbol{\theta}) & \mathbf{K}_{p}^{\gamma_{r} \boldsymbol{\gamma}_{1}}(\boldsymbol{\theta}) & \ldots & \mathbf{K}_{p}^{\boldsymbol{\gamma}_{r} \boldsymbol{\gamma}_{\boldsymbol{r}}}(\boldsymbol{\theta}) & \mathbf{K}_{p}^{\boldsymbol{\gamma}_{r} \phi}(\boldsymbol{\theta}) \\
\mathbf{K}_{p}^{\phi \boldsymbol{\beta}}(\boldsymbol{\theta}) & \mathbf{K}_{p}^{\phi \boldsymbol{\gamma}_{1}}(\boldsymbol{\theta}) & \ldots & \mathbf{K}_{p}^{\phi \boldsymbol{\gamma}_{r}}(\boldsymbol{\theta}) & \mathrm{K}_{p}^{\phi \phi}(\boldsymbol{\theta})
\end{array}\right)
$$

Vamos iniciar derivando a matriz de informação de Fisher $\mathbf{K}_{p}^{\boldsymbol{\beta} \boldsymbol{\beta}}(\boldsymbol{\theta})$. Temos que

$$
\mathrm{K}_{p}^{\beta_{j} \beta_{l}}(\boldsymbol{\theta})=E\left\{-\frac{\partial^{2} L_{p}(\boldsymbol{\theta}, \lambda)}{\partial \beta_{j} \partial \beta_{l}}\right\}, \text { para } j, l=1, \ldots, p .
$$

A segunda derivada da equação (B.2) em relação à $\boldsymbol{\beta}$, é dada por: 


$$
\begin{aligned}
\frac{\partial^{2} L_{p}(\boldsymbol{\theta}, \boldsymbol{\lambda})}{\partial \beta_{j} \partial \beta_{l}} & =\frac{\partial}{\partial \beta_{l}}\left[\phi \sum_{i=1}^{n}\left\{\frac{d \theta_{i}}{d \mu_{i}} \frac{d \mu_{i}}{d \eta_{i}}\left(y_{i}-\mu_{i}\right) x_{i j}\right\}\right]= \\
& =\phi \sum_{i=1}^{n} \frac{d^{2} \theta_{i}}{d \mu_{i}^{2}}\left(\frac{d \mu_{i}}{d \eta_{i}}\right)^{2} \frac{\partial \eta_{i}}{\partial \beta_{l}}\left(y_{i}-\mu_{i}\right) x_{i j}+\phi \sum_{i=1}^{n} \frac{d \theta_{i}}{d \mu_{i}} \frac{d^{2} \mu_{i}}{d \eta_{i}^{2}} \frac{\partial \eta_{i}}{\partial \beta_{l}}\left(y_{i}-\mu_{i}\right) x_{i j}- \\
& -\phi \sum_{i=1}^{n} \frac{d \theta_{i}}{d \mu_{i}}\left(\frac{d \mu_{i}}{d \eta_{i}}\right)^{2} \frac{\partial \eta_{i}}{\partial \beta_{l}} x_{i j}= \\
& =\phi \sum_{i=1}^{n} \frac{d^{2} \theta_{i}}{d \mu_{i}^{2}}\left(\frac{d \mu_{i}}{d \eta_{i}}\right)^{2}\left(y_{i}-\mu_{i}\right) x_{i j} x_{i l}+\phi \sum_{i=1}^{n} \frac{d \theta_{i}}{d \mu_{i}} \frac{d^{2} \mu_{i}}{d \eta_{i}^{2}}\left(y_{i}-\mu_{i}\right) x_{i j} x_{i l}- \\
& -\phi \sum_{i=1}^{n} \frac{d \theta_{i}}{d \mu_{i}}\left(\frac{d \mu_{i}}{d \eta_{i}}\right)^{2} x_{i j} x_{i l}, \text { para } j, l=1, \ldots, p .
\end{aligned}
$$

Sendo assim,

$$
\begin{aligned}
\mathrm{K}_{p}^{\beta_{j} \beta_{l}}(\boldsymbol{\theta}) & =E\left\{-\frac{\partial^{2} L_{p}(\boldsymbol{\theta}, \lambda)}{\partial \beta_{j} \partial \beta_{l}}\right\}= \\
& =E\left\{\phi \sum_{i=1}^{n} \frac{d \theta_{i}}{d \mu_{i}}\left(\frac{d \mu_{i}}{d \eta_{i}}\right)^{2} x_{i j} x_{i l}\right\}= \\
& =\phi \sum_{i=1}^{n} w_{i} x_{i j} x_{i l}, \text { para } j, l=1, \ldots, p .
\end{aligned}
$$

Escrevendo a equação acima em forma matricial, obtemos

$$
\mathbf{K}_{p}^{\boldsymbol{\beta} \boldsymbol{\beta}}(\boldsymbol{\theta})=\phi \mathbf{X}^{\top} \mathbf{W} \mathbf{X}
$$

A matriz de informação de Fisher penalizada para o parâmetro $\gamma_{l}$ da parte não paramétrica é formada pelos componentes

$$
\mathrm{K}_{p}^{\gamma_{j l} \gamma_{k l}}(\boldsymbol{\theta})=E\left\{-\frac{\partial^{2} L_{p}(\boldsymbol{\theta}, \lambda)}{\partial \gamma_{j l} \partial \gamma_{k l}}\right\} \text {, para } j, k=1, \ldots, q_{l} .
$$

Vamos inicialmente obter a segunda derivada da equação (B.3)

$$
\begin{aligned}
\frac{\partial^{2} L_{p}(\boldsymbol{\theta}, \boldsymbol{\lambda})}{\partial \gamma_{j l} \partial \gamma_{k l}} & =\frac{\partial}{\partial \gamma_{k l}}\left\{\phi \sum_{i=1}^{n} \frac{d \theta_{i}}{d \mu_{i}} \frac{d \mu_{i}}{d \eta_{i}}\left(y_{i}-\mu_{i}\right) b_{i j l}-\left[\lambda_{l} \mathbf{P}_{l}^{d} \boldsymbol{\gamma}_{l}\right]_{j}\right\}= \\
& =\phi \sum_{i=1}^{n} \frac{d^{2} \theta_{i}}{d \mu_{i}^{2}}\left(\frac{d \mu_{i}}{d \eta_{i}}\right)^{2} \frac{\partial \eta_{i}}{\partial \gamma_{k l}}\left(y_{i}-\mu_{i}\right) b_{i j l}+\phi \sum_{i=1}^{n} \frac{d \theta_{i}}{d \mu_{i}} \frac{d^{2} \mu_{i}}{d \eta_{i}^{2}} \frac{\partial \eta_{i}}{\partial \gamma_{k l}}\left(y_{i}-\mu_{i}\right) b_{i j l}- \\
& -\phi \frac{d \theta_{i}}{d \mu_{i}}\left(\frac{d \mu_{i}}{d \eta_{i}}\right)^{2} \frac{\partial \eta_{i}}{\partial \gamma_{k l}} b_{i j l}-\lambda_{l} p_{i j l}= \\
& =\phi \sum_{i=1}^{n} \frac{d^{2} \theta_{i}}{d \mu_{i}^{2}}\left(\frac{d \mu_{i}}{d \eta_{i}}\right)^{2}\left(y_{i}-\mu_{i}\right) b_{i j l} b_{i k l}+\phi \sum_{i=1}^{n} \frac{d \theta_{i}}{d \mu_{i}} \frac{d^{2} \mu_{i}}{d \eta_{i}^{2}}\left(y_{i}-\mu_{i}\right) b_{i j l} b_{i k l}- \\
& -\phi \frac{d \theta_{i}}{d \mu_{i}}\left(\frac{d \mu_{i}}{d \eta_{i}}\right)^{2} b_{i j l} b_{i k l}-\lambda_{l} p_{i j l}, \text { para } j, k=1, \ldots, q_{l} .
\end{aligned}
$$


Assim

$$
\begin{aligned}
\mathbf{K}_{p}^{\gamma_{j l} \gamma_{k l}}(\boldsymbol{\theta}) & =E\left\{-\frac{\partial^{2} L_{p}(\boldsymbol{\theta}, \boldsymbol{\lambda})}{\partial \gamma_{j l} \partial \gamma_{k l}}\right\}= \\
& =E\left\{\phi \sum_{i=1}^{n} \frac{d \theta_{i}}{d \mu_{i}}\left(\frac{d \mu_{i}}{d \eta_{i}}\right)^{2} b_{i j l} b_{i k l}\right\}+E\left\{\lambda_{l} p_{i j l}\right\}= \\
& =\phi \sum_{i=1}^{n} w_{i} b_{i j l} b_{i k l}+\lambda_{l} p_{i j l}, \text { para } j, k=1, \ldots, q_{l} .
\end{aligned}
$$

Escrevendo em forma matricial, obtemos

$$
\mathbf{K}_{p}^{\gamma_{l} \boldsymbol{\gamma}_{l}}(\boldsymbol{\theta})=\phi \mathbf{B}_{l}^{\top} \mathbf{W} \mathbf{B}_{l}+\lambda_{l} \mathbf{P}_{l}^{d},
$$

$\operatorname{com} l=1, \ldots, r$.

Para o parâmetro de precisão $\phi$, a informação de Fisher penalizada é definida por

$$
\mathrm{K}_{p}^{\phi \phi}(\boldsymbol{\theta})=E\left\{-\frac{\partial^{2} L_{p}(\boldsymbol{\theta}, \lambda)}{\partial \phi \partial \phi}\right\} .
$$

A segunda derivada da equação (B.4) em relação a $\phi$ fica dada por:

$$
\begin{aligned}
\frac{\partial^{2} L_{p}(\boldsymbol{\theta}, \boldsymbol{\lambda})}{\partial \phi \partial \phi} & =\frac{\partial}{\partial \phi}\left[\sum_{i=1}^{n}\left\{y_{i} \theta_{i}-b\left(\theta_{i}\right)\right\}+\sum_{i=1}^{n} c^{\prime}\left(y_{i} ; \phi\right)\right]= \\
& =\sum_{i=1}^{n} c^{\prime \prime}\left(y_{i} ; \phi\right) .
\end{aligned}
$$

Assim, obtemos

$$
\mathrm{K}_{p}^{\phi \phi}(\boldsymbol{\theta})=E\left\{-\frac{\partial^{2} L_{p}(\boldsymbol{\theta}, \boldsymbol{\lambda})}{\partial \phi \partial \phi}\right\}=-\sum_{i=1}^{n} E\left\{c^{\prime \prime}\left(y_{i} ; \phi\right)\right\},
$$

em que $c^{\prime \prime}\left(y_{i} ; \phi\right)=\frac{d^{2} c\left(y_{i} ; \phi\right)}{d \phi^{2}}$.

Vamos agora derivar as matrizes de informação de Fisher penalizadas dos parâmetros cruzados.

Começando pela matriz de informação de Fisher $\mathbf{K}_{p}^{\boldsymbol{\beta} \gamma_{l}}(\boldsymbol{\theta})$, temos que

$$
\mathrm{K}_{p}^{\beta_{j} \gamma_{k l}}(\boldsymbol{\theta})=E\left\{-\frac{\partial^{2} L_{p}(\boldsymbol{\theta}, \lambda)}{\partial \beta_{j} \partial \gamma_{k l}}\right\}
$$

para $j=1, \ldots, p, k=1, \ldots, q_{l}$ e $l=1, \ldots, r$.

A segunda derivada da equação (B.5) em relação à $\gamma$ fica dada por 


$$
\begin{aligned}
\frac{\partial^{2} L_{p}(\boldsymbol{\theta}, \boldsymbol{\lambda})}{\partial \beta_{j} \partial \gamma_{k l}} & =\frac{\partial}{\partial \gamma_{k l}}\left[\phi\left\{\sum_{i=1}^{n} \frac{d \theta_{i}}{d \mu_{i}} \frac{d \mu_{i}}{d \eta_{i}}\left(y_{i}-\mu_{i}\right) x_{i j}\right\}\right]= \\
& =\phi \sum_{i=1}^{n} \frac{d^{2} \theta_{i}}{d \mu_{i}^{2}}\left(\frac{d \mu_{i}}{d \eta_{i}}\right)^{2} \frac{\partial \eta_{i}}{\partial \gamma_{k l}}\left(y_{i}-\mu_{i}\right) x_{i j}+\phi \sum_{i=1}^{n} \frac{d \theta_{i}}{d \mu_{i}} \frac{d^{2} \mu_{i}}{d \eta_{i}^{2}} \frac{\partial \eta_{i}}{\partial \gamma_{k l}}\left(y_{i}-\mu_{i}\right) x_{i j}- \\
& -\phi \sum_{i=1}^{n} \frac{d \theta_{i}}{d \mu_{i}}\left(\frac{d \mu_{i}}{d \eta_{i}}\right)^{2} \frac{\partial \eta_{i}}{\partial \gamma_{k l}} x_{i j}= \\
& =\phi \sum_{i=1}^{n} \frac{d^{2} \theta_{i}}{d \mu_{i}^{2}}\left(\frac{d \mu_{i}}{d \eta_{i}}\right)^{2}\left(y_{i}-\mu_{i}\right) x_{i j} b_{i k l}+\phi \sum_{i=1}^{n} \frac{d \theta_{i}}{d \mu_{i}} \frac{d^{2} \mu_{i}}{d \eta_{i}^{2}}\left(y_{i}-\mu_{i}\right) x_{i j} b_{i k l}- \\
& -\phi \sum_{i=1}^{n} \frac{d \theta_{i}}{d \mu_{i}}\left(\frac{d \mu_{i}}{d \eta_{i}}\right)^{2} x_{i j} b_{i k l}, \text { para } j=1, \ldots, p, k=1, \ldots, q_{l} \text { e } l=1, \ldots, r .
\end{aligned}
$$

Assim, temos que

$$
\begin{aligned}
\mathrm{K}_{p}^{\beta_{j} \gamma_{k l}}(\boldsymbol{\theta}) & =E\left\{-\frac{\partial^{2} L_{p}(\boldsymbol{\theta}, \boldsymbol{\lambda})}{\partial \beta_{j} \partial \gamma_{k l}}\right\}= \\
& =E\left\{\phi \sum_{i=1}^{n} w_{i} x_{i j} b_{i k l}\right\}= \\
& =\phi \sum_{i=1}^{n} w_{i} x_{i j} b_{i k l}, \text { para } j=1, \ldots, p, k=1, \ldots, q_{l} \text { e } l=1, \ldots, r .
\end{aligned}
$$

Em forma matricial, escrevemos

$$
\mathbf{K}_{p}^{\boldsymbol{\beta} \gamma_{l}}(\boldsymbol{\theta})=\phi \mathbf{X}^{\top} \mathbf{W} \mathbf{B}_{l},
$$

para $l=1, \ldots, r$.

A informação de Fisher penalizada $K_{p}^{\beta_{j} \phi}(\boldsymbol{\theta})$ é dada por

$$
\mathrm{K}_{p}^{\beta_{j} \phi}(\boldsymbol{\theta})=E\left\{-\frac{\partial^{2} L_{p}(\boldsymbol{\theta}, \lambda)}{\partial \beta_{j} \partial \phi}\right\}, \text { para } j=1, \ldots, p .
$$

Calculando a segunda derivada de (B.5) em relação à $\phi$, temos que:

$$
\begin{aligned}
-\frac{\partial^{2} L_{p}(\boldsymbol{\theta}, \boldsymbol{\lambda})}{\partial \beta_{j} \partial \phi} & =-\frac{\partial}{\partial \phi}\left[\phi\left\{\sum_{i=1}^{n} \frac{d \theta_{i}}{d \mu_{i}} \frac{d \mu_{i}}{d \eta_{i}}\left(y_{i}-\mu_{i}\right) x_{i j}\right\}\right]= \\
& =-\sum_{i=1}^{n} \frac{d \theta_{i}}{d \mu_{i}} \frac{d \mu_{i}}{d \eta_{i}}\left(y_{i}-\mu_{i}\right) x_{i j}= \\
& =-\sum_{i=1}^{n} \sqrt{\frac{w_{i}}{V_{i}}}\left(y_{i}-\mu_{i}\right) x_{i j}, \text { para } j=1, \ldots, p .
\end{aligned}
$$

Com isso, obtemos o seguinte resultado:

$$
\begin{aligned}
\mathrm{K}_{p}^{\beta_{j} \phi}(\boldsymbol{\theta}) & =E\left\{-\frac{\partial^{2} L_{p}(\boldsymbol{\theta}, \boldsymbol{\lambda})}{\partial \beta_{j} \partial \phi}\right\}= \\
& =E\left\{-\sum_{i=1}^{n} \sqrt{\frac{w_{i}}{V_{i}}}\left(y_{i}-\mu_{i}\right) x_{i j}\right\}=0, \text { para } j=1, \ldots, p
\end{aligned}
$$


Para os parâmetros $\gamma_{j l}$ e $\gamma_{k l^{\prime}}$ temos que a informação observada penalizada é definida por

$$
\mathbf{K}_{p}^{\gamma_{j l} \gamma_{k l^{\prime}}}(\boldsymbol{\theta})=E\left\{-\frac{\partial^{2} L_{p}(\boldsymbol{\theta}, \lambda)}{\partial \gamma_{j l} \partial \gamma_{k l^{\prime}}}\right\}
$$

para $j, k=1, \ldots, q_{l}$ e $l, l^{\prime}=1, \ldots, r$.

A segunda derivada de (B.6) é dada por

$$
\begin{aligned}
\frac{\partial^{2} L_{p}(\boldsymbol{\theta}, \boldsymbol{\lambda})}{\partial \gamma_{j l} \partial \gamma_{k l^{\prime}}} & =\frac{\partial}{\partial \gamma_{k l^{\prime}}}\left\{\phi \sum_{i=1}^{n} \frac{d \theta_{i}}{d \mu_{i}} \frac{d \mu_{i}}{d \eta_{i}}\left(y_{i}-\mu_{i}\right) b_{i j l}-\left[\lambda_{l} \mathbf{P}_{l}^{d} \boldsymbol{\gamma}_{l}\right]_{j}\right\}= \\
& =\phi \sum_{i=1}^{n} \frac{d^{2} \theta_{i}}{d \mu_{i}^{2}}\left(\frac{d \mu_{i}}{d \eta_{i}}\right)^{2} \frac{\partial \eta_{i}}{\partial \gamma_{k l^{\prime}}}\left(y_{i}-\mu_{i}\right) b_{i j l}+\phi \sum_{i=1}^{n} \frac{d \theta_{i}}{d \mu_{i}} \frac{d^{2} \mu_{i}}{d \eta_{i}^{2}} \frac{\partial \eta_{i}}{\partial \gamma_{k l^{\prime}}}\left(y_{i}-\mu_{i}\right) b_{i j l}- \\
& -\phi \frac{d \theta_{i}}{d \mu_{i}}\left(\frac{d \mu_{i}}{d \eta_{i}}\right)^{2} \frac{\partial \eta_{i}}{\partial \gamma_{k l^{\prime}}} b_{i j l}= \\
& =\phi \sum_{i=1}^{n} \frac{d^{2} \theta_{i}}{d \mu_{i}^{2}}\left(\frac{d \mu_{i}}{d \eta_{i}}\right)^{2}\left(y_{i}-\mu_{i}\right) b_{i j l} b_{i k l^{\prime}}+\phi \sum_{i=1}^{n} \frac{d \theta_{i}}{d \mu_{i}} \frac{d^{2} \mu_{i}}{d \eta_{i}^{2}}\left(y_{i}-\mu_{i}\right) b_{i j l} b_{i k l^{\prime}}- \\
& -\phi \frac{d \theta_{i}}{d \mu_{i}}\left(\frac{d \mu_{i}}{d \eta_{i}}\right)^{2} b_{i j l} b_{i k l^{\prime}}, \text { para } j, k=1, \ldots, q_{l} .
\end{aligned}
$$

Assim, obtemos

$$
\begin{aligned}
\mathrm{K}_{p}^{\gamma_{j l} \gamma_{k l^{\prime}}}(\boldsymbol{\theta}) & =E\left\{-\frac{\partial^{2} L_{p}(\boldsymbol{\theta}, \boldsymbol{\lambda})}{\partial \gamma_{j l} \partial \gamma_{k l^{\prime}}}\right\}= \\
& =E\left\{\phi \sum_{i=1}^{n} \frac{d \theta_{i}}{d \mu_{i}}\left(\frac{d \mu_{i}}{d \eta_{i}}\right)^{2} b_{i j l} b_{i k l^{\prime}}\right\}= \\
& =\phi \sum_{i=1}^{n} w_{i} b_{i j l} b_{i k l^{\prime}}, \text { para } j, k=1, \ldots, q_{l} .
\end{aligned}
$$

Escrevendo em forma matricial, obtemos

$$
\mathbf{K}_{p}^{\gamma_{l} \gamma_{l^{\prime}}}(\boldsymbol{\theta})=\phi \mathbf{B}_{l}^{\top} \mathbf{W B}_{l^{\prime}}
$$

para $l, l^{\prime}=1, \ldots, r$.

Podemos reescrever (B.6) e (B.10) da seguinte maneira:

$$
\mathbf{K}_{p}^{\gamma_{l} \boldsymbol{\gamma}_{l^{\prime}}}(\boldsymbol{\theta})= \begin{cases}\phi \mathbf{B}_{l}^{\top} \mathbf{W} \mathbf{B}_{l}+\lambda_{l} \mathbf{P}_{l}^{d}, & \text { se } l=l^{\prime} \\ \phi \mathbf{B}_{l}^{\top} \mathbf{W B}_{l^{\prime}}, & \text { se } l \neq l^{\prime}\end{cases}
$$

$\operatorname{com} l, l^{\prime}=1, \ldots, r$.

Por fim, a informação de Fisher penalizada para os parâmetros $\gamma_{l}$ e $\phi$ fica dada por

$$
\mathrm{K}_{p}^{\gamma_{j l} \phi}(\boldsymbol{\theta})=E\left\{-\frac{\partial^{2} L_{p}(\boldsymbol{\theta}, \lambda)}{\partial \gamma_{j l} \partial \phi}\right\}
$$

para $j=1, \ldots, q_{l}$ e $l=1, \ldots, r$.

Calculando a segunda derivada da equação (B.6) com relação à $\phi$, temos que: 


$$
\begin{aligned}
\frac{\partial L_{p}(\boldsymbol{\theta}, \boldsymbol{\lambda})}{\partial \gamma_{j l} \partial \phi} & =\frac{\partial}{\partial \phi}\left[\phi\left\{\sum_{i=1}^{n} \frac{d \theta_{i}}{d \mu_{i}} \frac{d \mu_{i}}{d \eta_{i}}\left(y_{i}-\mu_{i}\right) b_{i j l}\right\}-\left[\lambda_{l} \mathbf{P}_{l}^{d} \boldsymbol{\gamma}_{l}\right]_{j}\right]= \\
& =\sum_{i=1}^{n} \frac{d \theta_{i}}{d \mu_{i}} \frac{d \mu_{i}}{d \eta_{i}}\left(y_{i}-\mu_{i}\right) b_{i j l}= \\
& =\sum_{i=1}^{n} \sqrt{\frac{w_{i}}{V_{i}}}\left(y_{i}-\mu_{i}\right) b_{i j l}, \text { para } j=1, \ldots, q_{l} \text { e } l=1, \ldots, r .
\end{aligned}
$$

Assim,

$$
\begin{aligned}
\mathrm{K}_{p}^{\gamma_{j l} \phi}(\boldsymbol{\theta}) & =E\left\{-\frac{\partial L_{p}(\boldsymbol{\theta}, \lambda)}{\partial \gamma_{j l} \partial \phi}\right\}= \\
& =E\left\{-\sum_{i=1}^{n} \sqrt{\frac{w_{i}}{V_{i}}}\left(y_{i}-\mu_{i}\right) b_{i j l}\right\}=0 .
\end{aligned}
$$


APÊNDICE B 


\section{Apêndice C}

\section{Derivação do Suavizador}

Vamos provar neste apêndice que na convergência do processo iterativo temos, para os MLPGs e MLPAGs, respectivamente,

$$
\hat{\boldsymbol{\eta}}=\widehat{\mathbf{H}}(\lambda) \hat{\mathbf{z}}
$$

e

$$
\hat{\boldsymbol{\eta}}=\widehat{\mathbf{H}}(\boldsymbol{\lambda}) \hat{\mathbf{z}}
$$

em que, nos MLPAGs, $\boldsymbol{\lambda}=\left(\lambda_{1}, \ldots, \lambda_{r}\right)^{\top}$.

\section{C.1 MLPGs}

Para os MLPGs, na convergência do processo iterativo, temos que

$$
\hat{\boldsymbol{\eta}}=\mathbf{X} \hat{\boldsymbol{\beta}}+\mathbf{B} \hat{\boldsymbol{\gamma}}=\mathbf{N} \hat{\boldsymbol{\xi}}=\widehat{\mathbf{H}}(\lambda) \hat{\mathbf{z}}
$$

em que $\mathbf{N} \boldsymbol{\xi}=[\mathbf{X}, \mathbf{B}] \boldsymbol{\xi}, \operatorname{com} \boldsymbol{\xi}=\left(\boldsymbol{\beta}^{\top}, \boldsymbol{\gamma}^{\top}\right)^{\top}$ e $\mathbf{z}=\mathbf{N} \boldsymbol{\xi}+\mathbf{W}^{-\frac{1}{2}} \mathbf{V}^{-\frac{1}{2}}(\mathbf{y}-\boldsymbol{\mu})$.

Note que

$$
\begin{gathered}
\mathbf{U}_{p}^{\boldsymbol{\xi}}(\boldsymbol{\theta})=\phi \mathbf{N}^{\top} \mathbf{W}^{\frac{1}{2}} \mathbf{V}^{-\frac{1}{2}}(\mathbf{y}-\boldsymbol{\mu})-\mathbf{P}(\lambda) \boldsymbol{\xi} \mathrm{e} \\
\mathbf{K}_{p}^{\boldsymbol{\xi} \boldsymbol{\xi}}(\boldsymbol{\theta})=\phi \mathbf{N}^{\top} \mathbf{W} \mathbf{N}+\mathbf{P}(\lambda),
\end{gathered}
$$

$\operatorname{com} \mathbf{P}(\lambda)=$ blocodiag $\left\{\mathbf{O}_{p p}, \lambda \mathbf{P}_{d}\right\}$, em que $\mathbf{O}_{p p}$ é uma matriz de zeros de dimensão $p \times p$.

Na convergência do processo iterativo temos que $\mathbf{U}_{p}^{\boldsymbol{\xi}}(\hat{\boldsymbol{\theta}})=0$, assim

$$
\hat{\phi} \mathbf{N}^{\top} \widehat{\mathbf{W}}^{\frac{1}{2}} \widehat{\mathbf{V}}^{-\frac{1}{2}}(\mathbf{y}-\hat{\boldsymbol{\mu}})=\mathbf{P}(\lambda) \hat{\boldsymbol{\xi}}
$$

Além disso, temos a seguinte igualdade:

$$
\begin{aligned}
& \hat{\boldsymbol{\xi}}=\left[\mathbf{K}_{p}^{\xi \boldsymbol{\xi}}(\hat{\boldsymbol{\theta}})\right]^{-1} \mathbf{K}_{p}^{\boldsymbol{\xi} \boldsymbol{\xi}}(\hat{\boldsymbol{\theta}}) \hat{\boldsymbol{\xi}} \\
& =\left[\hat{\phi} \mathbf{N}^{\top} \widehat{\mathbf{W}} \mathbf{N}+\mathbf{P}(\lambda)\right]^{-1} \mathbf{K}_{p}^{\xi \boldsymbol{\xi}}(\hat{\boldsymbol{\theta}}) \hat{\boldsymbol{\xi}},
\end{aligned}
$$


em que, utilizando o resultado (C.1), obtemos

$$
\begin{aligned}
\mathbf{K}_{p}^{\xi \boldsymbol{\xi}}(\hat{\boldsymbol{\theta}}) \hat{\boldsymbol{\xi}} & =\hat{\phi} \mathbf{N}^{\top} \widehat{\mathbf{W}} \mathbf{N} \hat{\boldsymbol{\xi}}+\mathbf{P}(\lambda) \hat{\boldsymbol{\xi}} \\
& =\hat{\phi} \mathbf{N}^{\top} \widehat{\mathbf{W}} \mathbf{N} \hat{\boldsymbol{\xi}}+\hat{\phi} \mathbf{N}^{\top} \widehat{\mathbf{W}} \widehat{\mathbf{V}}^{\frac{1}{2}} \widehat{\mathbf{V}}^{-\frac{1}{2}}(\mathbf{y}-\hat{\boldsymbol{\mu}}) \\
& =\hat{\phi} \mathbf{N}^{\top} \widehat{\mathbf{W}}\left\{\mathbf{N} \hat{\boldsymbol{\xi}}+\widehat{\mathbf{W}} \frac{1}{2} \widehat{\mathbf{V}}^{-\frac{1}{2}}(\mathbf{y}-\hat{\boldsymbol{\mu}})\right\} \\
& =\hat{\phi} \mathbf{N}^{\top} \widehat{\mathbf{W}} \hat{\mathbf{z}},
\end{aligned}
$$

$\operatorname{com} \hat{\mathbf{z}}=\mathbf{N} \hat{\boldsymbol{\xi}}+\widehat{\mathbf{W}}^{-\frac{1}{2}} \widehat{\mathbf{V}}^{-\frac{1}{2}}(\mathbf{y}-\hat{\boldsymbol{\mu}})$.

Sendo assim, temos que

$$
\begin{aligned}
\hat{\boldsymbol{\xi}} & =\left[\hat{\phi} \mathbf{N}^{\top} \widehat{\mathbf{W}} \mathbf{N}+\mathbf{P}(\lambda)\right]^{-1} \mathbf{K}_{p}^{\boldsymbol{\xi} \boldsymbol{\xi}}(\hat{\boldsymbol{\theta}}) \hat{\boldsymbol{\xi}} \\
& =\left[\mathbf{N}^{\top} \widehat{\mathbf{W}} \mathbf{N}+\hat{\phi}^{-1} \mathbf{P}(\lambda)\right]^{-1} \mathbf{N}^{\top} \widehat{\mathbf{W}} \hat{\mathbf{z}} .
\end{aligned}
$$

Desta forma, podemos escrever $\hat{\boldsymbol{\eta}}$ como

$$
\begin{aligned}
\hat{\eta} & =\mathbf{N} \hat{\boldsymbol{\xi}}=\mathbf{N}\left[\mathbf{N}^{\top} \widehat{\mathbf{W}} \mathbf{N}+\hat{\phi}^{-1} \mathbf{P}(\lambda)\right]^{-1} \mathbf{N}^{\top} \widehat{\mathbf{W}} \hat{\mathbf{z}} \\
& =\mathbf{N}\left[\mathbf{N}^{\top} \widehat{\mathbf{W}} \mathbf{N}+\mathbf{P}(\lambda)\right]^{-1} \mathbf{N}^{\top} \widehat{\mathbf{W}} \hat{\mathbf{z}} \\
& =\widehat{\mathbf{H}}(\lambda) \hat{\mathbf{z}},
\end{aligned}
$$

em que redefinimos o parâmetro de suavização para $\lambda=\lambda \phi^{-1}$, fazendo com que o $\phi$ desaparecesse da expressão acima.

\section{C.2 MLPAGs}

Na convergência do processo iterativo temos que, para os MLPAGs,

$$
\hat{\boldsymbol{\eta}}=\mathbf{X} \hat{\boldsymbol{\beta}}+\sum_{l=1}^{r} \mathbf{B}_{l} \hat{\gamma}_{l}=\mathbf{N} \hat{\boldsymbol{\xi}}=\widehat{\mathbf{H}}(\boldsymbol{\lambda}) \hat{\mathbf{z}}
$$

em que $\mathbf{N} \boldsymbol{\xi}=\left[\mathbf{X}, \mathbf{B}_{1}, \ldots, \mathbf{B}_{r}\right] \boldsymbol{\xi}, \operatorname{com} \boldsymbol{\xi}=\left(\boldsymbol{\beta}^{\top}, \boldsymbol{\gamma}^{\top}\right)^{\top}, \boldsymbol{\gamma}=\left(\boldsymbol{\gamma}_{1}^{\top}, \ldots, \boldsymbol{\gamma}_{r}^{\top}\right)^{\top}$ e $\mathbf{z}=\mathbf{N} \boldsymbol{\xi}+\mathbf{W}^{-\frac{1}{2}} \mathbf{V}^{-\frac{1}{2}}(\mathbf{y}-$ $\boldsymbol{\mu})$.

A função escore e a matriz de informação de Fisher penalizadas são dadas por

$$
\begin{gathered}
\mathbf{U}_{p}^{\boldsymbol{\xi}}(\boldsymbol{\theta})=\phi \mathbf{N}^{\top} \mathbf{W}^{\frac{1}{2}} \mathbf{V}^{-\frac{1}{2}}(\mathbf{y}-\boldsymbol{\mu})-\mathbf{P}(\boldsymbol{\lambda}) \boldsymbol{\xi} \mathrm{e} \\
\mathbf{K}_{p}^{\boldsymbol{\xi} \boldsymbol{\xi}}(\boldsymbol{\theta})=\phi \mathbf{N}^{\top} \mathbf{W} \mathbf{N}+\mathbf{P}(\boldsymbol{\lambda})
\end{gathered}
$$

$\operatorname{com} \mathbf{P}(\lambda)=$ blocodiag $\left\{\mathbf{O}_{p p}, \lambda_{1} \mathbf{P}_{1}^{d}, \ldots, \lambda_{r} \mathbf{P}_{r}^{d}\right\}$, em que $\mathbf{O}_{p p}$ é uma matriz de zeros de dimensão $p \times p$.

$\mathrm{Na}$ convergência do processo iterativo temos que $\mathbf{U}_{p}^{\boldsymbol{\xi}}(\hat{\boldsymbol{\theta}})=0$, assim

$$
\hat{\phi} \mathbf{N}^{\top} \widehat{\mathbf{W}}^{\frac{1}{2}} \widehat{\mathbf{V}}^{-\frac{1}{2}}(\mathbf{y}-\hat{\boldsymbol{\mu}})=\mathbf{P}(\boldsymbol{\lambda}) \hat{\boldsymbol{\xi}}
$$

Além disso, podemos escrever $\hat{\boldsymbol{\xi}}$ da seguinte maneira:

$$
\begin{aligned}
& \hat{\boldsymbol{\xi}}=\left[\mathbf{K}_{p}^{\xi \boldsymbol{\xi}}(\hat{\boldsymbol{\theta}})\right]^{-1} \mathbf{K}_{p}^{\boldsymbol{\xi} \boldsymbol{\xi}}(\hat{\boldsymbol{\theta}}) \hat{\boldsymbol{\xi}} \\
& =\left[\hat{\phi} \mathbf{N}^{\top} \widehat{\mathbf{W}} \mathbf{N}+\mathbf{P}(\boldsymbol{\lambda})\right]^{-1} \mathbf{K}_{p}^{\boldsymbol{\xi} \boldsymbol{\xi}}(\hat{\boldsymbol{\theta}}) \hat{\boldsymbol{\xi}},
\end{aligned}
$$


em que, utilizando o resultado (C.2), temos

$$
\begin{aligned}
\mathbf{K}_{p}^{\xi \boldsymbol{\xi}}(\hat{\boldsymbol{\theta}}) \hat{\boldsymbol{\xi}} & =\hat{\phi} \mathbf{N}^{\top} \widehat{\mathbf{W}} \mathbf{N} \hat{\boldsymbol{\xi}}+\mathbf{P}(\boldsymbol{\lambda}) \hat{\boldsymbol{\xi}} \\
& =\hat{\phi} \mathbf{N}^{\top} \widehat{\mathbf{W}} \mathbf{N} \hat{\boldsymbol{\xi}}+\hat{\phi} \mathbf{N}^{\top} \widehat{\mathbf{W}} \widehat{\mathbf{V}}^{\frac{1}{2}} \widehat{\mathbf{V}}^{-\frac{1}{2}}(\mathbf{y}-\hat{\boldsymbol{\mu}}) \\
& =\hat{\phi} \mathbf{N}^{\top} \widehat{\mathbf{W}}\left\{\mathbf{N} \hat{\boldsymbol{\xi}}+\widehat{\mathbf{W}} \frac{1}{2} \widehat{\mathbf{V}}^{-\frac{1}{2}}(\mathbf{y}-\hat{\boldsymbol{\mu}})\right\} \\
& =\hat{\phi} \mathbf{N}^{\top} \widehat{\mathbf{W}} \hat{\mathbf{z}},
\end{aligned}
$$

$\operatorname{com} \hat{\mathbf{z}}=\mathbf{N} \hat{\boldsymbol{\xi}}+\widehat{\mathbf{W}}^{-\frac{1}{2}} \widehat{\mathbf{V}}^{-\frac{1}{2}}(\mathbf{y}-\hat{\boldsymbol{\mu}})$.

Sendo assim, obtemos

$$
\begin{aligned}
\hat{\boldsymbol{\xi}} & =\left[\hat{\phi} \mathbf{N}^{\top} \widehat{\mathbf{W}} \mathbf{N}+\mathbf{P}(\boldsymbol{\lambda})\right]^{-1} \mathbf{K}_{p}^{\boldsymbol{\xi} \boldsymbol{\xi}}(\hat{\boldsymbol{\theta}}) \hat{\boldsymbol{\xi}} \\
& =\left[\mathbf{N}^{\top} \widehat{\mathbf{W}} \mathbf{N}+\hat{\phi}^{-1} \mathbf{P}(\boldsymbol{\lambda})\right]^{-1} \mathbf{N}^{\top} \widehat{\mathbf{W}} \hat{\mathbf{z}},
\end{aligned}
$$

de forma que podemos escrever $\hat{\boldsymbol{\eta}}$ como

$$
\begin{aligned}
\hat{\eta} & =\mathbf{N} \hat{\boldsymbol{\xi}}=\mathbf{N}\left[\mathbf{N}^{\top} \widehat{\mathbf{W}} \mathbf{N}+\hat{\phi}^{-1} \mathbf{P}(\boldsymbol{\lambda})\right]^{-1} \mathbf{N}^{\top} \widehat{\mathbf{W}} \hat{\mathbf{z}} \\
& =\mathbf{N}\left[\mathbf{N}^{\top} \widehat{\mathbf{W}} \mathbf{N}+\mathbf{P}(\boldsymbol{\lambda})\right]^{-1} \mathbf{N}^{\top} \widehat{\mathbf{W}} \hat{\mathbf{z}} \\
& =\widehat{\mathbf{H}}(\boldsymbol{\lambda}) \hat{\mathbf{z}},
\end{aligned}
$$

em que $\phi$ desaparece da expressão acima ao redefinirmos o parâmetro de suavização para $\lambda_{l}=\lambda_{l} \phi^{-1}$, $\operatorname{com} l=1, \ldots, r$. 
APÊNDICE C 


\section{Referências Bibliográficas}

Akaike(1974) Hirotugu Akaike. A new look at the statistical model identification. IEEE Transactions on Automatic Control, AC-19(6):716-723. Citado na pág. 31

Aranda-Ordaz(1981) Francisco J. Aranda-Ordaz. On two families of transformations to additivity for binary response data. Biometrika, 68(2):357-363. Citado na pág. 17

Box e Cox(1964) G.E.P. Box e D.R. Cox. An analysis of transformation. Journal of the Royal Statistical Society, 26:211-252. Citado na pág. 17

Breiman e Friedman(1985) Leo Breiman e Jerome H. Friedman. Estimating optimal transformations for multiple regression and correlation. Journal of the American Statistical Association, 80:580-298. Citado na pág. 27

Buja et al.(1989) Andreas Buja, Trevor Hastie e Robert Tibshirani. Linear smoother and additive models. The Annals of Statistics, 17:453-555. Citado na pág. 27

Craven e Wahba(1979) Peter Craven e Grace Wahba. Smoothing noisy data with spline functions. Numerical Mathematical, 31:377-403. Citado na pág. 30

De Boor(1978) Carl De Boor. A Practical Guide to Splines. Springer, Berlin. Citado na pág. 2, 18

Dunn e Smyth(1996) Peter K. Dunn e Gordon K. Smyth. Randomized quantile residuals. Journal of Computational and Graphical Statistics, 5:236-244. Citado na pág. 33

Eilers e Marx(2010) Paul H. C. Eilers e Brian D. Marx. Splines, knots and penalties. Wiley Interdisciplinary Reviews: Computational Statistics, 2(6):637-653. Citado na pág. 24

Eilers e Marx(1996) Paul H. C. Eilers e Brian D. Marx. Flexible smoothing with B-splines and penalties. Statistical Science, 11(2):89-121. Citado na pág. 2, 20, 21, 24, 29

Eilers et al.(2015) Paul H. C. Eilers, Brian D. Marx e Maria Durbán. Twenty years of P-splines. Statistics and Operations Research Transactions, 39(2):149-186. Citado na pág. 24

Eubank(1999) Randall L. Eubank. Nonparametric Regression and Spline Smoothing. Marcel Dekker, $2^{0}$ edição. Citado na pág. 1, 2, 29

Fahrmeir e Tutz(2001) Ludwig Fahrmeir e Gerhard Tutz. Multivariate Statistical Modelling Based on Generalized Linear Models. Springer, $2^{\circ}$ edição. Citado na pág. 15

Green(1987) Peter J. Green. Penalized likelihood for general semi-parametric regression models. International Statistical Review, 55:245-259. Citado na pág. 24

Green e Yandell(1985) Peter J. Green e Brian S. Yandell. Semi-parametric generalized linear models. Lecture Notes in Statistics, 32:44-55. Citado na pág. 15

Green e Silverman(1994) P.J. Green e B.W. Silverman. Nonparametric Regression and Generalized Linear Models: a Roughness Penalty Approach. Chapman and Hall. Citado na pág. 2, 15, 23, 25, 28, 29, 30, 40, 65 
Gu(2013) Chong Gu. Smoothing Spline ANOVA Models. Springer-Verlag New York, $2^{\circ}$ edição. Citado na pág. 27

Hastie e Tibshirani(1990) T. J. Hastie e R. J. Tibshirani. Generalized Additive Models. Chapman and Hall, London. Citado na pág. 2, 28, 29, 35, 42

Hubert e Vandervieren(2008) Mia Hubert e Ellen Vandervieren. An adjusted boxplot for skewed distributions. Computational Statistics and Data Analysis, 52(12):5186-5201. Citado na pág. 5

Ibacache-Pulgar(2009) Germán Mauricio Ibacache-Pulgar. Modelos Mistos Aditivos Semiparamétricos de Contornos Elípticos. Tese de Doutorado, Instituto de Matemática e Estatística da Universidade de São Paulo. Citado na pág. 24

Marx e Eilers(1998) Brian D. Marx e Paul H. C. Eilers. Direct generalized additive modelling with penalized likelihood. Computacional Statistics and Data Analysis, 28:103-209. Citado na pág. 36

McCullagh e Nelder(1989) P. McCullagh e J. A. Nelder. Generalized Linear Models. Chapman and Hall, $2^{\circ}$ edição. Citado na pág. 17

Milicer e Szczotka(1965) Halina Milicer e Franciszek Szczotka. Age at menarche in Warsaw girls in 1965. Human Biology, 38(3):199-203. Citado na pág. 3

Neter et al.(1996) J. Neter, M. H. Kutner, J. C. Nachtsheim e W. Wasserman. Applied Linear Regression Models. McGraw-Hill/Irwin, $3^{\circ}$ edição. Citado na pág. 4

Noda(2013) Gleyce Rocha Noda. Análise de Diagnóstico em Modelos Semiparamétricos Normais. Dissertação de Mestrado, Instituto de Matemática e Estatística da Universidade de São Paulo. Citado na pág. 29

Paula(2013) Gilberto Alvarenga Paula. Modelos de Regressão com Apoio Computacional. Instituto de Matemática e Estatística da Universidade de São Paulo. Citado na pág. 3, 17

Reinsch(1967) Christian H. Reinsch. Smoothing by spline functions. Numerische Mathematik, 10:177-183. Citado na pág. 2, 23

Ribgy e Stasinopoulos(2005) Robert A. Ribgy e Mikis D. Stasinopoulos. Generalized additive models for location, scale and shape. Journal of the Royal Statistical Society, 54:507-554. Citado na pág. $3,35,45$

Ruppert(2002) David Ruppert. Selecting the number of knots for penalized splines. Journal of Computational and Graphical Statistics, 11(4):735-757. Citado na pág. 19, 22

Schwarz(1978) Gideon Schwarz. Estimating the dimension of a model. The Annals of Statistics, 6(2):461-464. Citado na pág. 31

Stasinopoulos e Ribgy(2007) Mikis D. Stasinopoulos e Robert A. Ribgy. Generalized additive models for location scale and shape (GAMLSS) in R. Journal of Statistical Software, 23. Citado na pág. 35,45

Vanegas e Paula(2016) Luis Hernando Vanegas e Gilberto A. Paula. An extension of logsymmetric regression models: $\mathrm{R}$ codes and applications. Journal of Statistical Simulation and Computation, 86:1709-1735. Citado na pág. 19, 32, 33, 37, 41, 43, 63

Wahba(1985) Grace Wahba. A comparison of GCV and GML for choosing the smoothing parameter in the generalized spline smoothing problem. The Annals of Statistics, 13(4):1378-1402. Citado na pág. 31 
Wahba e Wold(1975) Grace Wahba e Svante Wold. A completely automatic french curve: Fitting spline functions by cross validation. Communications in Statistics, 4:1-17. Citado na pág. 30

Wand e Jones(1995) M. P. Wand e M. C. Jones. Kernel Smoothing. Chapman and Hall. Citado na pág. 1

Wand e Ormerod(2008) M. P. Wand e J. T. Ormerod. On semiparametric regression with O'Sullivan penalized splines. Australian and New Zealand Journal of Statistics, 50(2):179-198. Citado na pág. 66

Wood(2017) Simon N. Wood. Generalized Additive Models: an Introduction with $R$. CRC Press, $2^{\circ}$ edição. Citado na pág. $2,30,32,33,35,37,41,44$

Yu e Ruppert(2002) Yan Yu e David Ruppert. Penalized spline estimation for partially linear single-index models. Journal of the American Statistical Association, 97:1042-1054. Citado na pág. 19,32 